\title{
Redox-Annulation of Cyclic Amines and $\beta$-Ketoaldehydes
}

\author{
Weijie Chen and Daniel Seidel ${ }^{*}$ \\ Department of Chemistry and Chemical Biology \\ Rutgers, The State University of New Jersey, Piscataway, New Jersey 08854, USA
}

\section{Supporting Information}

General Information: Starting materials, reagents, and solvents were purchased from commercial sources and used as received unless stated otherwise. 1,2,3,4-Tetrahydroisoquinoline (THIQ), acetic acid and 2-ethylhexanoic acid (2-EHA) were distilled prior to use. Benzoic acid was recrystallized from toluene/ethanol. Purification of reaction products was carried out by flash column chromatography using Sorbent Technologies Standard Grade silica gel (60 ̊, 230-400 mesh). Analytical thin layer chromatography was performed on EM Reagent $0.25 \mathrm{~mm}$ silica gel $60 \mathrm{~F}_{254}$ plates. Visualization was accomplished with UV light, potassium permanganate or Dragendorff-Munier stains, followed by heating. Melting points were recorded on a Thomas Hoover capillary melting point apparatus and are uncorrected. Infrared spectra were recorded on an ATI Mattson Genesis Series FT-Infrared spectrophotometer. Proton nuclear magnetic resonance spectra $\left({ }^{1} \mathrm{H}-\mathrm{NMR}\right)$ were recorded on a Varian VNMRS-500 MHz, Varian VNMRS-400 MHz or Varian VNMRS-300 MHz and chemical shifts are reported in ppm using the solvent as an internal standard $\left(\mathrm{CDCl}_{3}\right.$ at $\left.7.26 \mathrm{ppm}\right)$. Data are reported as app $=$ apparent, $\mathrm{s}=$ singlet, $\mathrm{d}=$ doublet, $\mathrm{t}=$ triplet, $\mathrm{q}=$ quartet, $\mathrm{m}=$ multiplet, comp = complex, $\mathrm{br}$ = broad; coupling constant(s) in Hz. Proton-decoupled carbon nuclear magnetic resonance spectra $\left({ }^{13} \mathrm{C}-\mathrm{NMR}\right)$ spectra were recorded on a Varian VNMRS-500 MHz, Varian VNMRS-400 MHz or Varian VNMRS-300 MHz and chemical shifts are reported in ppm using the solvent as an internal standard $\left(\mathrm{CDCl}_{3}\right.$ at $\left.77.0 \mathrm{ppm}\right)$. Mass spectra were recorded on a Finnigan LCQ-DUO mass spectrometer. Ratios of diastereomeric products were determined by ${ }^{1} \mathrm{H}-\mathrm{NMR}$ analysis of the crude reaction mixture. 6,7-Methylenedioxy-1,2,3,4-tetrahydroisoquinoline, ${ }^{1}$ 9-methyltryptoline, ${ }^{2}$ 2,2-dimethyl-3oxobutyraldehyde, ${ }^{3} \quad$ 1-acetylcyclohexanecarbaldehyde, ${ }^{4} \quad$ 2,2-dimethyl-3-oxo-4-phenylbutyraldehyde, ${ }^{5}$ 4-benzyloxy-1-bromobutane, ${ }^{6}$ and 3-ethylpentane-2,4-dione ${ }^{7}$ were prepared according to literature procedures. Compounds $\mathbf{1 n},{ }^{8} \mathbf{4 a - 4 d},{ }^{9} \mathbf{6 a},{ }^{10}$ and $\mathbf{6} \mathbf{d}^{11}$ were previously reported and their published characterization data matched our own in all respects. 
Scheme S1: Synthesis of 2-alkyl-3-oxobutyraldehydes. ${ }^{12,13}$
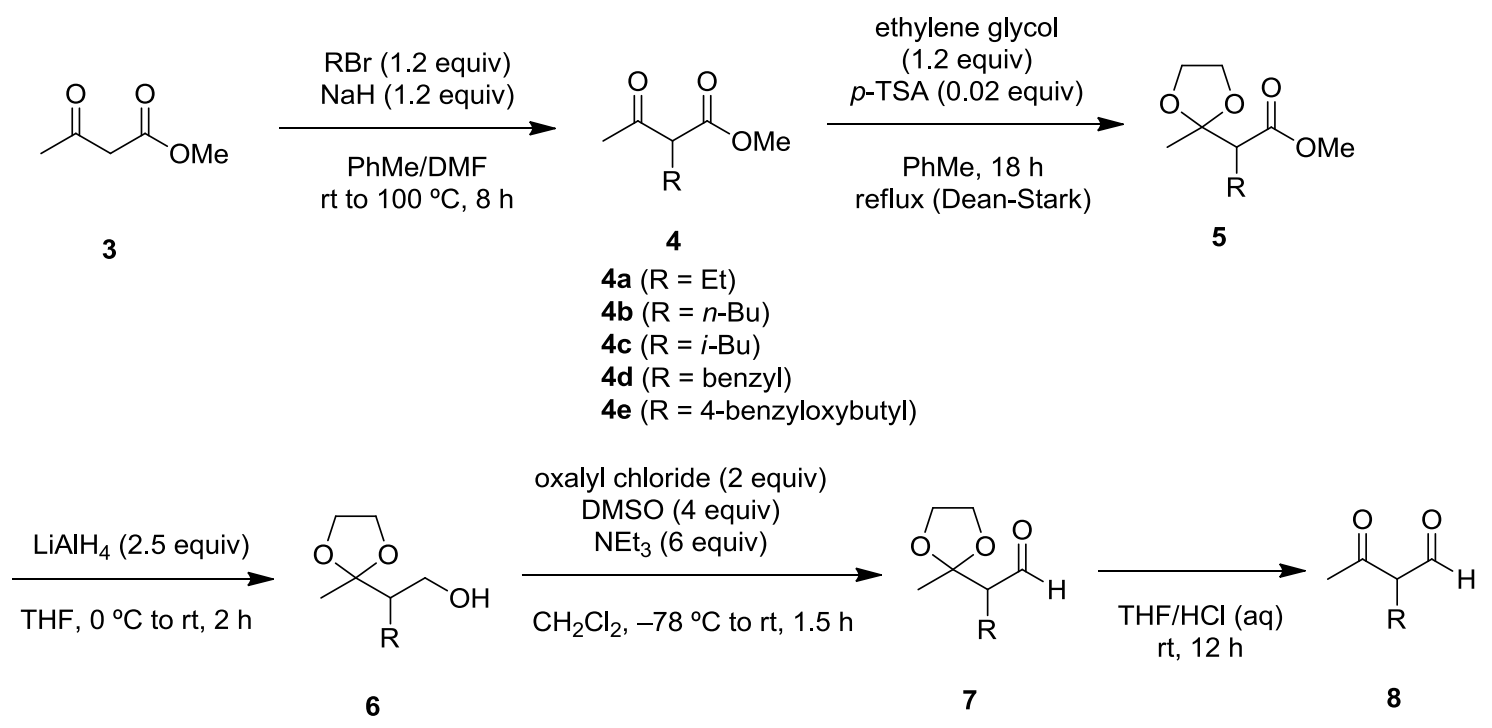

methyl 2-acetyl-6-(benzyloxy)hexanoate (4e): To a suspension of $\mathrm{NaH}(60 \%$ in mineral<smiles>COC(=O)C(CCCCOc1ccccc1)C(C)=O</smiles>
oil, $0.96 \mathrm{~g}, 24 \mathrm{mmol}, 1.2$ equiv) in a mixture of dry toluene $(12 \mathrm{~mL})$ and DMF $(10 \mathrm{~mL})$ was slowly added methyl acetoacetate $(2.16 \mathrm{~mL}, 20 \mathrm{mmol}, 1$ equiv) over 20 minutes at room temperature. The mixture was then allowed to stir for 15 minutes at room temperature before benzyloxy-1-bromobutane $(5.83 \mathrm{~g}$, $24 \mathrm{mmol}, 1.2$ equiv) was added in one portion. The reaction mixture was allowed to stir at $100{ }^{\circ} \mathrm{C}$ for 8 hours. After cooling to room temperature, the reaction was quenched by the addition of saturated aqueous $\mathrm{NH}_{4} \mathrm{Cl}$. $6 \mathrm{M} \mathrm{HCl}(5 \mathrm{~mL})$ was then added to the reaction mixture. The reaction mixture was washed with water $(2 \times 75 \mathrm{~mL})$, and the combined aqueous layers were extracted with ethyl ether $(3 \times 50 \mathrm{~mL})$. The combined organic layers were then washed with brine $(150 \mathrm{~mL})$ and dried over anhydrous $\mathrm{Na}_{2} \mathrm{SO}_{4}$. Solvent was then removed under reduced pressure. The residue was purified by silica gel chromatography and compound $4 \mathbf{e}$ was obtained as a yellow oil in $77 \%$ yield $(4.29 \mathrm{~g}),\left(\mathrm{R}_{\mathrm{f}}=\right.$ 0.22 in hexanes/EtOAc 90:10 v/v); IR (KBr) 2951, 2861, 1742, 1716, 1643, 1496, 1455, 1433, 1360, 1245, 1208, 1151, 1095, 737, $699 \mathrm{~cm}^{-1} ;{ }^{1} \mathrm{H}$ NMR $\left(500 \mathrm{MHz}, \mathrm{CDCl}_{3}\right) 7.37-7.29$ (comp, 4H), 7.29-7.24 (m, 1H), 4.51-4.45 (comp, 2H), 3.72 (s, 3H), 3.48-3.40 (comp, 3H), 2.21 (s, 3H), 1.92-1.79 (comp, 2H), 1.67-1.57 (comp, 2H), 1.42-1.31 (comp, 2H); ${ }^{13} \mathrm{C}$ NMR $\left(125 \mathrm{MHz}, \mathrm{CDCl}_{3}\right) \delta 203.0,170.2,138.5,128.3,127.6,127.5,72.9,69.8,59.6,52.3$, 29.4, 28.8, 28.0, 24.1; $\mathrm{m} / z$ (ESI-MS) $301.2[\mathrm{M}+\mathrm{Na}]^{+}$.

\section{General Procedure A for converting 4 to 5:}

A mixture of 2-alkyl methyl acetoacetate (20 mmol, 1 equiv), ethylene glycol (1.34 $\mathrm{mL}, 24$ mmol, 1.2 equiv) and $p$-TSA monohydrate $(76 \mathrm{mg}, 0.4 \mathrm{mmol}, 0.02$ equiv) in toluene ( $30 \mathrm{~mL})$ was heated under reflux with a Dean-Stark apparatus for 18 hours. The reaction mixture was allowed to cool to room temperature and then diluted with ethyl ether $(30 \mathrm{~mL})$. The mixture was washed with saturated aqueous $\mathrm{NaHCO}_{3}(3 \times 30 \mathrm{~mL})$, and the combined 
aqueous layers were washed with ethyl ether $(3 \times 30 \mathrm{~mL})$. The combined organic layers were washed with brine and dried over anhydrous $\mathrm{Na}_{2} \mathrm{SO}_{4}$. Solvent was then removed under reduced pressure and the residue was further dried under high vacuum. The crude product was directly used in the next step without further purification.

\section{General Procedure B for converting 5 to 6:}

To an ice-cooled suspension of $\mathrm{LiAlH}_{4}(1.43 \mathrm{~g}, 37.5 \mathrm{mmol}, 2.5$ equiv) in dry THF (60 mL) was slowly added a solution of compound 5 (15 mmol, 1 equiv) in dry THF (15 mL). The mixture was allowed to stir at room temperature for 2 hours, followed by quenching with $30 \%$ aqueous ammonia cooled in an ice bath. The lithium and aluminum hydroxide salts were then filtered through a short pad of celite and washed with EtOAc $(6 \times 50 \mathrm{~mL})$. The solvent was then removed under reduced pressure, and the residue was purified by silica gel chromatography.

New compounds were characterized as below:

2-(2-methyl-1,3-dioxolan-2-yl)hexan-1-ol (6b): Following the general procedures A and B compound $\mathbf{6 b}$ was obtained from compound $\mathbf{4 b}$ as a colorless oil in $61 \%$ yield over two steps $(1.72 \mathrm{~g}),\left(\mathrm{R}_{\mathrm{f}}=0.34\right.$ in hexanes/EtOAc 70:30 v/v); IR $(\mathrm{KBr})$

${ }_{n-\mathrm{Bu}} \quad 3385,2956,2935,2866,2357,2330,1701,1650,1458,1369,1085,1041,882$
${ }^{-1}$; $\mathrm{H}$ NMR (500 MHz, $\mathrm{CDCl}_{3}$ ) 3.99-3.94 (comp, 4H), 3.67-3.58 (comp, 2H), 2.96 (br s, $\mathrm{cm}^{-1}$; ${ }^{1} \mathrm{H}$ NMR $\left(500 \mathrm{MHz}, \mathrm{CDCl}_{3}\right)$ 3.99-3.94 (comp, 4H), 3.67-3.58 (comp, 2H), 2.96 (br s,
$1 \mathrm{H}), 1.79-1.72(\mathrm{~m}, 1 \mathrm{H}), 1.51-1.21(\mathrm{comp}, 8 \mathrm{H}), 1.17-1.08(\mathrm{~m}, 1 \mathrm{H}), 0.88(\mathrm{t}, J=7.0 \mathrm{~Hz}, 3 \mathrm{H})$; ${ }^{13} \mathrm{C}$ NMR $\left(125 \mathrm{MHz}, \mathrm{CDCl}_{3}\right) \delta 113.1,64.5,64.2,62.7,47.8,30.2,26.6,22.9,20.5,13.9$; $m / z\left(\right.$ ESI-MS) $167.0\left[\mathrm{M}+\mathrm{Na}-\mathrm{C}_{2} \mathrm{H}_{4} \mathrm{O}\right]^{+}$.

4-methyl-2-(2-methyl-1,3-dioxolan-2-yl)pentan-1-ol (6c): Following the general $\mathrm{C}^{\mathrm{O}}$ procedures $\mathrm{A}$ and $\mathrm{B}$ compound $\mathbf{6 c}$ was obtained from compound $\mathbf{4 c}$ as a colorless oil in $64 \%$ yield over two steps $(1.81 \mathrm{~g}),\left(\mathrm{R}_{\mathrm{f}}=0.34\right.$ in hexanes/EtOAc 70:30 v/v); IR (KBr) 3385, 2957, 2935, 2873, 1701, 1643, 1465, 1418, 1369, 1247, 1208, 1166, 1085, 1041, $882 \mathrm{~cm}^{-1} ;{ }^{1} \mathrm{H}$ NMR $\left(500 \mathrm{MHz}, \mathrm{CDCl}_{3}\right)$ 4.02-3.92 (comp, 4H), 3.63-3.57 (comp, 2H), 3.10 (br s, 1H), 1.89-1.83 (m, 1H), 1.68-1.57 (m, 1H), 1.29 (s, 3H), 1.89 (ddd, $J=13.7,9.7,3.2 \mathrm{~Hz}, 1 \mathrm{H}), 1.08$ (ddd, $J=13.7,9.9,4.6 \mathrm{~Hz}$, 1H), 0.92-0.85 (comp, 6H); ${ }^{13} \mathrm{C}$ NMR (125 MHz, $\left.\mathrm{CDCl}_{3}\right) \delta 113.2,64.5,64.2,63.1,45.4$, $36.2,25.9,23.8,21.7,20.5 ; \quad m / z$ (ESI-MS) $167.0\left[\mathrm{M}+\mathrm{Na}-\mathrm{C}_{2} \mathrm{H}_{4} \mathrm{O}\right]^{+}$.

6-(benzyloxy)-2-(2-methyl-1,3-dioxolan-2-yl)hexan-1-ol (6e): Following the general

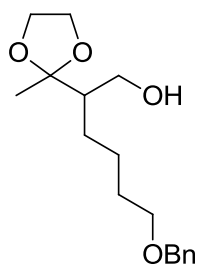
procedures $\mathrm{A}$ and $\mathrm{B}$ compound $\mathbf{6 e}$ was obtained from compound $4 \mathbf{e}$ as a colorless oil in $70 \%$ yield over two steps $(3.09 \mathrm{~g}),\left(\mathrm{R}_{\mathrm{f}}=0.26\right.$ in hexanes/EtOAc $70: 30 \mathrm{v} / \mathrm{v}) ; \quad \mathrm{IR}(\mathrm{KBr}) 3422,2938,2863,2360,2337,1647,1453,1364,1098$, 1040, 946, $860 \mathrm{~cm}^{-1}$; ${ }^{1} \mathrm{H}$ NMR (500 MHz, $\mathrm{CDCl}_{3}$ ) 7.37-7.32 (comp, 4H), $7.30-7.25$ (m, 1H), 4.50 (s, 2H), 4.00-3.92 (comp, 4H), 3.69-3.59 (comp, 2H), 3.47 (app t, $J=6.6 \mathrm{~Hz}, 2 \mathrm{H}), 3.00$ (br s, 1H), 1.81-1.75 (m, 1H), 1.73-1.44 (comp, 4H), 1.43-1.33 (m, 1H), $1.30(\mathrm{~s}, 3 \mathrm{H}), 1.21-1.12(\mathrm{~m}, 1 \mathrm{H}) ;{ }^{13} \mathrm{C}$ NMR $(125 \mathrm{MHz}$, 
$\left.\mathrm{CDCl}_{3}\right) \delta 138.6,128.3,127.6,127.5,113.0,72.9,70.2,64.5,64.2,62.7,47.9,30.0,26.8,24.7$, $20.5 ; \quad m / z\left(\right.$ ESI-MS) $317.2[\mathrm{M}+\mathrm{Na}]^{+}$.

\section{General Procedure $\mathrm{C}$ for converting 6 to 7:}

To a stirred solution of oxalyl chloride ( $1.71 \mathrm{~mL}, 20 \mathrm{mmol}, 2$ equiv) in dry $\mathrm{CH}_{2} \mathrm{Cl}_{2}(20 \mathrm{~mL})$ was slowly added dry DMSO $\left(2.84 \mathrm{~mL}, 40 \mathrm{mmol}, 4\right.$ equiv) at $-78{ }^{\circ} \mathrm{C}$. The mixture was allowed to stir at the same temperature for 15 minutes, then a solution of the alcohol $\mathbf{6}$ (10 mmol, 1 equiv) in $\mathrm{CH}_{2} \mathrm{Cl}_{2}(25 \mathrm{~mL})$ was slowly added. After stirring at $-78{ }^{\circ} \mathrm{C}$ for 30 minutes, $\mathrm{NEt}_{3}(8.36 \mathrm{~mL}, 60 \mathrm{mmol}, 6$ equiv) was slowly added and the reaction mixture was allowed to warm to room temperature and stirred for another hour. The reaction mixture was diluted with ethyl ether $(50 \mathrm{~mL})$ and washed with water $(3 \times 50 \mathrm{~mL})$. The combined aqueous layers were extracted with ethyl ether $(3 \times 50 \mathrm{~mL})$. The combined organic layers were then washed with brine and dried over anhydrous $\mathrm{Na}_{2} \mathrm{SO}_{4}$. Solvent was then removed under reduced pressure and the crude product was directly used in the next step without further purification.

\section{General Procedure D for converting 7 to 8:}

The crude compound 7 (10 mmol) was dissolved in a mixture of THF (15 mL) and $3 \mathrm{M} \mathrm{HCl}$ $(10 \mathrm{~mL})$. The mixture was allowed to stir at room temperature for 12 hours. The reaction mixture was then diluted with ethyl ether $(20 \mathrm{~mL})$ and washed with water $(3 \times 30 \mathrm{~mL})$. The combined aqueous layers were extracted with ethyl ether $(3 \times 30 \mathrm{~mL})$. The combined organic layers were then washed with brine and dried over anhydrous $\mathrm{Na}_{2} \mathrm{SO}_{4}$. Solvent was then removed under reduced pressure and the residue was purified by Kugelrohr distillation $(\mathbf{8 a}-\mathbf{c})$, recrystallization from EtOAc/hexanes $(\mathbf{8 d})$ or silica gel chromatography (8e).

New compounds were characterized as below:

2-ethyl-3-oxobutanal (8a): Following the general procedures $\mathrm{C}$ and D compound 8a was o obtained from compound $\mathbf{6 a}$ as a white solid in $85 \%$ yield over two steps $(0.97 \mathrm{~g})$, $\left(\mathrm{R}_{\mathrm{f}}=0.39\right.$ in hexanes/EtOAc 90:10 v/v); mp: 39-41 ${ }^{\circ} \mathrm{C} ; \quad \mathrm{IR}(\mathrm{KBr}) 2972,2925$, 2871, 1634, 1435, 1366, 1258, 1212, 1117, 1071, 1024, $912 \mathrm{~cm}^{-1} ; \quad{ }^{1} \mathrm{H}$ NMR (500

$\left.\mathrm{MHz}, \mathrm{CDCl}_{3}\right) 7.86$ (br s, 1H), 2.18 (q, $\left.J=7.5 \mathrm{~Hz}, 2 \mathrm{H}\right), 2.14$ (s, 3H), 1.05 (t, $J=7.5 \mathrm{~Hz}, 3 \mathrm{H}$ );

${ }^{13} \mathrm{C} \mathrm{NMR}\left(125 \mathrm{MHz}, \mathrm{CDCl}_{3}\right) \delta 195.7,175.5,114.4,24.0,21.0,15.4 ; \quad \mathrm{m} / \mathrm{z}$ (ESI-MS) 169.1 $[\mathrm{M}+\mathrm{Na}+\mathrm{MeOH}]^{+}$.

2-acetylhexanal (8b): Following the general procedures $\mathrm{C}$ and $\mathrm{D}$ compound $\mathbf{8 b}$ was O obtained from compound $\mathbf{6 b}$ as a yellow oil in $87 \%$ yield over two steps $(1.24 \mathrm{~g})$, H $\left(\mathrm{R}_{\mathrm{f}}=0.56\right.$ in hexanes/EtOAc 80:20 v/v); IR (KBr) 2958, 2930, 2862, 2686, ${ }_{n-\mathrm{Bu}} 1718,1617,1406,1354,1277,1216,1126,1026,961,919 \mathrm{~cm}^{-1} ; \quad{ }^{1} \mathrm{H}$ NMR $(500$ $\left.\mathrm{MHz}, \mathrm{CDCl}_{3}\right) 7.93(\mathrm{~d}, J=6.8 \mathrm{~Hz}, 1 \mathrm{H}), 2.17-2.11$ (comp, 5H), 1.42-1.27 (comp, 4H), 0.91 (t, $J=7.2 \mathrm{~Hz}, 3 \mathrm{H}) ;{ }^{13} \mathrm{C} \mathrm{NMR}\left(125 \mathrm{MHz}, \mathrm{CDCl}_{3}\right) \delta 194.7,177.0,112.9,33.1,27.4,23.7,22.1$, 13.8; $\mathrm{m} / z(\mathrm{ESI}-\mathrm{MS}) 142.7[\mathrm{M}+\mathrm{H}]^{+}$. 
2-acetyl-4-methylpentanal (8c): Following the general procedures $\mathrm{C}$ and D compound 8c O was obtained from compound $\mathbf{6 c}$ as a white solid in $86 \%$ yield over two steps H $(1.23 \mathrm{~g}),\left(\mathrm{R}_{\mathrm{f}}=0.56\right.$ in hexanes/EtOAc 80:20 v/v); mp: 49-52 ${ }^{\circ} \mathrm{C} ; \mathrm{IR}(\mathrm{KBr})$ 2958, 2866, 2364, 2340, 1705, 1637, 1408, 1235, 1129, $973 \mathrm{~cm}^{-1} ; \quad{ }^{1} \mathrm{H}$ NMR (300 $\left.\mathrm{MHz}, \mathrm{CDCl}_{3}\right) 8.00(\mathrm{~d}, J=5.5 \mathrm{~Hz}, 1 \mathrm{H}), 2.12(\mathrm{~s}, 3 \mathrm{H}), 2.02(\mathrm{~d}, J=7.2 \mathrm{~Hz}, 2 \mathrm{H})$, $1.69-1.54(\mathrm{~m}, 1 \mathrm{H}), 0.90(\mathrm{~d}, J=6.8 \mathrm{~Hz}, 6 \mathrm{H}) ;{ }^{13} \mathrm{C} \mathrm{NMR}\left(75 \mathrm{MHz}, \mathrm{CDCl}_{3}\right) \delta 193.6,179.3$, $111.7,36.8,29.3,23.5,22.0 ; \quad \mathrm{m} / z$ (ESI-MS) $142.7[\mathrm{M}+\mathrm{H}]^{+}$.

2-benzyl-3-oxobutanal (8d): Following the general procedures $\mathrm{C}$ and $\mathrm{D}$ compound 8d was O obtained from compound $\mathbf{6 d}$ as a white solid in $90 \%$ yield over two steps $(1.58 \mathrm{~g})$, H $\left(\mathrm{R}_{\mathrm{f}}=0.38\right.$ in hexanes/EtOAc 80:20 v/v); $\mathrm{mp}: 98-101{ }^{\circ} \mathrm{C} ; \quad \mathrm{IR}(\mathrm{KBr}) 3111,2672$, Ph 1962, 1836, 1652, 1559, 1496, 1390, 1275, 1193, 1076, 1023, 973, 841, 808, 795, 726, 701, 654, 615, $526 \mathrm{~cm}^{-1} ;{ }^{1} \mathrm{H} \mathrm{NMR}\left(500 \mathrm{MHz}, \mathrm{CDCl}_{3}\right) 7.87(\mathrm{~d}, J=7.4 \mathrm{~Hz}, 1 \mathrm{H})$, 7.35-7.28 (comp, 2H), 7.23 (app t, $J=7.5 \mathrm{~Hz}, 1 \mathrm{H}$ ), 7.20-7.16 (comp, 2H), 3.54 (s, 2H), 2.04 $(\mathrm{s}, 3 \mathrm{H}) ;{ }^{13} \mathrm{C} \mathrm{NMR}\left(125 \mathrm{MHz}, \mathrm{CDCl}_{3}\right) \delta 197.3,174.9,139.3,128.3,127.6,126.1,111.4$, 33.4, 24.7; (ESI-MS) $199.0[\mathrm{M}+\mathrm{Na}]^{+}, 231.0[\mathrm{M}+\mathrm{Na}+\mathrm{MeOH}]^{+}$.

2-acetyl-6-(benzyloxy)hexanal (8e): Following the general procedures $\mathrm{C}$ and $\mathrm{D}$ compound

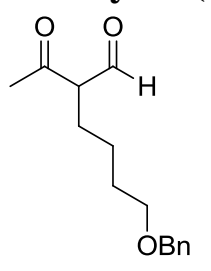

8e was obtained from compound $\mathbf{6 e}$ as a yellow oil in $93 \%$ yield $(2.31 \mathrm{~g}),\left(\mathrm{R}_{\mathrm{f}}=\right.$ 0.18 in hexanes/EtOAc 90:10 v/v); IR (KBr) 3064, 3030, 2936, 2861, 2792, 2695, 1707, 1617, 1496, 1454, 1406, 1362, 1265, 1212, 1103, 1028, 959, 907, 737, $699 \mathrm{~cm}^{-1} ;{ }^{1} \mathrm{H}$ NMR $\left(500 \mathrm{MHz}, \mathrm{CDCl}_{3}\right) 7.94(\mathrm{~d}, J=6.8 \mathrm{~Hz}, 1 \mathrm{H})$, 7.38-7.25 (comp, 5H), 4.50 (s, 2H), $3.49(\mathrm{t}, J=6.1 \mathrm{~Hz}, 2 \mathrm{H}), 2.18(\mathrm{t}, J=7.7 \mathrm{~Hz}$, $2 \mathrm{H}), 2.13(\mathrm{~s}, 3 \mathrm{H}), 1.68-1.60(\mathrm{~m}, 2 \mathrm{H}), 1.55-1.47(\mathrm{~m}, 2 \mathrm{H}) ;{ }^{13} \mathrm{C} \mathrm{NMR}\left(100 \mathrm{MHz}, \mathrm{CDCl}_{3}\right) \delta$ 194.7, 177.1, 138.4, 128.4, 127.6(1), 127.5(6), 112.6, 73.0, 69.9, 29.2, 27.6, 27.5, 23.8; $\mathrm{m} / \mathrm{z}$ (ESI-MS) $249.0[\mathrm{M}+\mathrm{H}]^{+}$.

\section{General Procedure for the Redox-Annulation Involving Non-enolizable $\beta$-Ketoaldehydes:}

To a mixture of the aldehyde $(0.5 \mathrm{mmol}, 1$ equiv) and $4 \AA \mathrm{MS}(150 \mathrm{mg})$ in toluene $(2 \mathrm{~mL})$ were added acetic acid $(0.29 \mathrm{~mL}, 5 \mathrm{mmol}, 10$ equiv) and the amine (0.75 mmol, 1.5 equiv). The mixture was heated under reflux for 2 hours. The reaction mixture was then allowed to cool to room temperature and filtered through a short pad of celite and washed with EtOAc (6 x $5 \mathrm{~mL}$ ). The filtrate was then washed with saturated aqueous $\mathrm{NaHCO}_{3}(3 \times 10 \mathrm{~mL})$. The combined aqueous layers were extracted with EtOAc $(3 \times 10 \mathrm{~mL})$, and the combined organic layers were washed with water $(40 \mathrm{~mL})$, brine $(40 \mathrm{~mL})$, and dried over anhydrous $\mathrm{Na}_{2} \mathrm{SO}_{4}$. Solvent was then removed under reduced pressure and the residue was purified by silica gel chromatography. 


\section{General Procedure for the Redox-Annulation Involving Enolizable $\beta$-Ketoaldehydes:}

To a suspension of $4 \AA \mathrm{MS}(150 \mathrm{mg})$ in toluene $(2 \mathrm{~mL})$ were added acetic acid $(0.29 \mathrm{~mL}, 5$ mmol, 10 equiv) and the amine $(0.75 \mathrm{mmol}, 1.5$ equiv). The mixture was heated under reflux and a solution of the aldehyde $(0.5 \mathrm{mmol}, 1$ equiv) in toluene was delivered through the top of the reflux condenser over 15 hours via syringe pump. The reaction was stopped immediately after the slow addition was completed. The reaction mixture was then allowed to cool to room temperature and filtered through a short pad of celite and washed with EtOAc (6 x $5 \mathrm{~mL})$. The filtrate was then washed with saturated aqueous $\mathrm{NaHCO}_{3}(3 \times 10 \mathrm{~mL})$. The combined aqueous layers were extracted with EtOAc $(3 \times 10 \mathrm{~mL})$, and the combined organic layers were washed with water $(40 \mathrm{~mL})$, brine $(40 \mathrm{~mL})$, and dried over anhydrous $\mathrm{Na}_{2} \mathrm{SO}_{4}$. Solvent was then removed under reduced pressure and the residue was purified by silica gel chromatography.

\section{3,3-dimethyl-3,4,6,7-tetrahydro-1H-pyrido[2,1-a]isoquinolin-2(11bH)-one}

(1a):

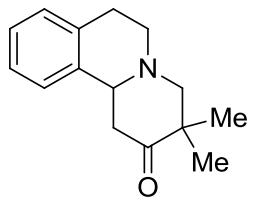

Following the general procedure compound 1a was obtained from THIQ (95 $\mu \mathrm{L})$ and 2,2-dimethyl-3-oxobutyraldehyde $(57 \mathrm{mg})$ as a yellow oil in $71 \%$ yield $(82 \mathrm{mg}),\left(\mathrm{R}_{\mathrm{f}}=0.47\right.$ in hexanes/EtOAc 90:10 v/v); IR $(\mathrm{KBr}) 3063$, 3022, 2961, 2923, 2805, 2758, 1713, 1633, 1495, 1469, 1453, 1427, 1377, $1355,1300,1248,1188,1145,1133,1112,1035,956,770,745,729,693 \mathrm{~cm}^{-1} ; \quad{ }^{1} \mathrm{H}$ NMR $\left(400 \mathrm{MHz} \mathrm{CDCl}_{3}\right)$ 7.24-7.12 (comp, 3H), 7.12-7.06 (m, 1H), 3.52-3.44 (m, 1H), 3.24 (ddd, $J=15.7,11.8,5.7 \mathrm{~Hz}, 1 \mathrm{H}), 3.03(\mathrm{ddd}, J=11.3,5.8,1.2 \mathrm{~Hz}, 1 \mathrm{H}), 2.88(\mathrm{dd}, J=14.6,3.3 \mathrm{~Hz}$, $1 \mathrm{H}), 2.82(\mathrm{~d}, J=11.5 \mathrm{~Hz}, 1 \mathrm{H}), 2.79-2.72(\mathrm{~m}, 1 \mathrm{H}), 2.68(\mathrm{dd}, J=14.6,12.1 \mathrm{~Hz}, 1 \mathrm{H}), 2.54$ (app td, $J=11.8,3.5 \mathrm{~Hz}, 1 \mathrm{H}), 2.45(\mathrm{~d}, J=11.5 \mathrm{~Hz}, 1 \mathrm{H}), 1.34(\mathrm{~s}, 3 \mathrm{H}), 1.08(\mathrm{~s}, 3 \mathrm{H}) ;{ }^{13} \mathrm{C}$ NMR $\left(100 \mathrm{MHz}, \mathrm{CDCl}_{3}\right) \delta 213.2,136.9,134.3,128.9,126.4,126.0,124.6,67.9,62.8,51.3$, 46.0, 44.3, 29.7, 25.7, 21.5; $\mathrm{m} / \mathrm{z}(\mathrm{ESI}-\mathrm{MS}) 230.2[\mathrm{M}+\mathrm{H}]^{+}, 262.0[\mathrm{M}+\mathrm{H}+\mathrm{MeOH}]^{+}$.

\section{9,10-dimethoxy-3,3-dimethyl-3,4,6,7-tetrahydro-1H-pyrido[2,1-a]isoquinolin-2(11bH)-o}

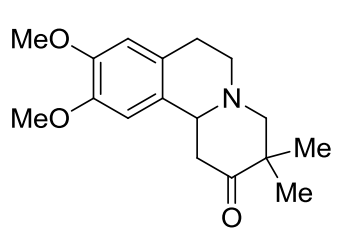

ne (1b): Following the general procedure compound 1b was obtained from 6,7-dimethoxy-1,2,3,4-tetrahydroisoquinoline (145 $\mathrm{mg})$ and 2,2-dimethyl-3-oxobutyraldehyde $(57 \mathrm{mg})$ as a yellow solid in $86 \%$ yield $(124 \mathrm{mg}),\left(\mathrm{R}_{\mathrm{f}}=0.56\right.$ in hexanes/EtOAc 60:40 v/v); $\mathrm{mp}$ : 127-128 ${ }^{\circ} \mathrm{C}$; IR (KBr) 3383, 2957, 2928, 2863, 2836, 2816, 2777, 2762, 2246, 1702, 1611, 1522, 1454, 1379, 1361, 1261, 1227, 1150, 1106, 1021, 919, 867, 88, 741, 711, $575 \mathrm{~cm}^{-1} ; \quad{ }^{1} \mathrm{H}$ NMR $\left(300 \mathrm{MHz}, \mathrm{CDCl}_{3}\right) 6.59(\mathrm{~s}, 1 \mathrm{H}), 6.53(\mathrm{~s}, 1 \mathrm{H}), 3.82(\mathrm{~s}, 3 \mathrm{H})$, 3.79 (s, 3H), 3.41-3.29 (m, 1H), 3.20-3.04 (m, 1H), 2.97 (ddd, $J=11.2,5.7,1.1 \mathrm{~Hz}, 1 \mathrm{H})$, 2.85-2.72 (comp, 2H), 2.70-2.55 (comp, 2H), 2.53-2.35 (comp, 2H), 1.29 (s, 3H), 1.03 (s, $3 \mathrm{H}) ;{ }^{13} \mathrm{C} \mathrm{NMR}\left(75 \mathrm{MHz}, \mathrm{CDCl}_{3}\right) \delta 213.2,147.5,147.3,128.6,126.3,111.3,107.6,67.7$, $62.4,55.8,55.7,51.4,45.9,44.5,29.2,25.6,21.4 ; \quad m / z$ (ESI-MS) $290.2[\mathrm{M}+\mathrm{H}]^{+}, 322.0[\mathrm{M}$ $+\mathrm{H}+\mathrm{MeOH}]^{+}$. 
3,3-dimethyl-3,4,6,7-tetrahydro-1H-[1,3]dioxolo[4,5-g]pyrido[2,1-a]isoquinolin-2(12bH)-

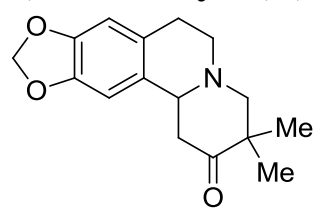

one (1c): Following the general procedure compound 1c was obtained from 6,7-methylenedioxy-1,2,3,4-tetrahydroisoquinoline $(133 \mathrm{mg})$ and 2,2-dimethyl-3-oxobutyraldehyde (57 $\mathrm{mg}$ ) as a yellow solid in $69 \%$ yield

$(94 \mathrm{mg}),\left(\mathrm{R}_{\mathrm{f}}=0.46\right.$ in hexanes/EtOAc $\left.80: 20 \mathrm{v} / \mathrm{v}\right) ; \mathrm{mp}: 121-123{ }^{\circ} \mathrm{C}$;

IR (KBr) 3382, 2947, 2879, 2808, 1860, 1705, 1507, 1483, 1380, 1355, 1327, 1292, 1233, 1139, 1039, 934, 867, 820, 773, 743, $546 \mathrm{~cm}^{-1}$; ${ }^{1} \mathrm{H}$ NMR $\left(300 \mathrm{MHz}, \mathrm{CDCl}_{3}\right) 6.56(\mathrm{~s}, 1 \mathrm{H})$, 6.53 (s. 1H), 5.91-5.86 (comp, 2H), 3.41-3.28 (m, 1H), 3.19-3.04 (m, 1H), 3.02-2.92 (m, 1H), 2.84-2.68 (comp, 2H), 2.67-2.54 (comp, 2H), 2.53-2.33 (comp, 2H), 1.30 (s, 3H), 1.04 $(\mathrm{s}, 3 \mathrm{H}) ; \quad{ }^{13} \mathrm{C} \mathrm{NMR}\left(75 \mathrm{MHz}, \mathrm{CDCl}_{3}\right) \delta 213.0,146.0(8), 146.0(7), 129.7,127.6,108.4,104.7$, $100.7,67.7,62.7,51.4,45.9,44.5,29.7,25.6,21.5 ; \quad \mathrm{m} / z$ (ESI-MS) $274.2[\mathrm{M}+\mathrm{H}]^{+}, 306.0$ $[\mathrm{M}+\mathrm{H}+\mathrm{MeOH}]^{+}$.

\section{3,3-dimethyl-1,3,4,6,7,12b-hexahydroindolo[2,3-a]quinolizin-2(12H)-one}

(1d):

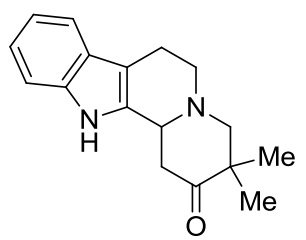

Following the general procedure compound 1d was obtained from tryptoline (129 $\mathrm{mg})$ and 2,2-dimethyl-3-oxobutyraldehyde $(57 \mathrm{mg})$ as a yellow solid in $67 \%$ yield $(90 \mathrm{mg}),\left(\mathrm{R}_{\mathrm{f}}=0.37\right.$ in hexanes/EtOAc 80:20 $\mathrm{v} / \mathrm{v})$; $\mathrm{mp}: 193-194{ }^{\circ} \mathrm{C}$; $\quad \mathrm{IR}(\mathrm{KBr}) 3059,2965,2921,2846,1705,1623$, $1467,1455,1383,1370,1352,1324,1283,12231,1173,1162,1121$, 1005, 961, 798, 739, 709, 667, $625 \mathrm{~cm}^{-1} ;{ }^{1} \mathrm{H} \mathrm{NMR}$ (500 MHz, $\mathrm{CDCl}_{3}$ ) 8.36 (br s, 1H), 7.54 (d, $J=7.7 \mathrm{~Hz}, 1 \mathrm{H}), 7.37$ (d, $J=7.9 \mathrm{~Hz}, 1 \mathrm{H}), 7.24-7.10$ (comp, 2H), 3.61-3.48 (m, 1H), 3.16 (dd, $J=10.9,5.4 \mathrm{~Hz}, 1 \mathrm{H}), 3.12-3.00$ (m, 1H), 2.98-2.73 (comp, 4H), 2.65 (app td, $J=11.1$, $3.4 \mathrm{~Hz}, 1 \mathrm{H}), 2.55(\mathrm{~d}, J=11.5 \mathrm{~Hz}, 1 \mathrm{H}), 1.39(\mathrm{~s}, 3 \mathrm{H}), 1.15(\mathrm{~s}, 3 \mathrm{H}) ;{ }^{13} \mathrm{C}$ NMR $(125 \mathrm{MHz}$, $\left.\mathrm{CDCl}_{3}\right) \delta 213.3,136.2,133.3,126.9,121.6,119.5,118.1,111.1,108.4,67.2,59.5,52.1,46.5$, 42.7, 25.8, 21.7(3), 21.6(9); $\mathrm{m} / z(\mathrm{ESI}-\mathrm{MS}) 269.2[\mathrm{M}+\mathrm{H}]^{+}, 301.1[\mathrm{M}+\mathrm{H}+\mathrm{MeOH}]^{+}$.

\section{3,3-dimethyl-11b-phenyl-3,4,6,7-tetrahydro-1H-pyrido[2,1-a]isoquinolin-2(11bH)-one}

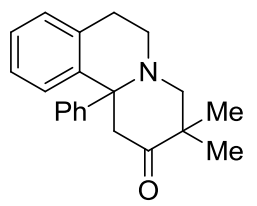

(1e): Following the general procedure compound 1e was obtained from 1-phenyl-THIQ (157 $\mathrm{mg}$ ) and 2,2-dimethyl-3-oxobutyraldehyde $(57 \mathrm{mg})$ as a yellow solid in $51 \%$ yield $(78 \mathrm{mg}),\left(\mathrm{R}_{\mathrm{f}}=0.64\right.$ in hexanes/EtOAc 80:20 v/v); mp: 101-104 ${ }^{\circ} \mathrm{C}$; IR (KBr) 3061, 3022, 2960, 2908, 2846, 1969, 1954, 1929, 1895, 1813, 1698, 1596, 1443, 1393, 1372, 1347, 1300, 1167, 1128, 958, 765, 745, 707 $\mathrm{cm}^{-1} ; \quad{ }^{1} \mathrm{H}$ NMR $\left(500 \mathrm{MHz}, \mathrm{CDCl}_{3}\right.$ ) 7.28-7.22 (comp, 2H), 7.22-7.07 (comp, 6H), 7.01 (d, J $=7.7 \mathrm{~Hz}, 1 \mathrm{H}), 3.27-3.21(\mathrm{~m}, 1 \mathrm{H}), 3.21-3.06$ (comp, 3H), 3.00-2.93 (m, 1H), 2.82-2.75 (m, 1H), 2.68-2.60 (comp, 2H), 1.25-1.17 (comp, 6H); ${ }^{13} \mathrm{C}$ NMR (125 MHz, $\left.\mathrm{CDCl}_{3}\right) \delta 214.4$, 144.6, 140.0, 134.0, 129.0, 128.1, 127.9, 127.7, 126.8, 126.5, 126.1, 66.3, 61.0, 49.1, 46.2, 46.0, 27.7, 25.3, 23.1; $\quad m / z(E S I-M S) 306.2[\mathrm{M}+\mathrm{H}]^{+}$.

$1^{\prime}, 6^{\prime}, 7^{\prime}, 11 b^{\prime}$-tetrahydrospiro[cyclohexane-1,3'-pyrido[2,1-a]isoquinolin]-2'(4'H)-one (1f):

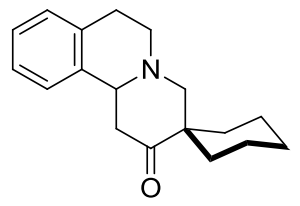
Following the general procedure compound 1f was obtained from THIQ $(95 \mu \mathrm{L})$ and 1-acetylcyclohexanecarbaldehyde $(77 \mathrm{mg})$ as a yellow oil in $71 \%$ yield $(96 \mathrm{mg}),\left(\mathrm{R}_{\mathrm{f}}=0.53\right.$ in hexanes/EtOAc 90:10 v/v); IR $(\mathrm{KBr})$ 3063, 3022, 2922, 2854, 2801, 2754, 1708, 1632, 1494, 1452, 1426, 1364, $1300,1275,1241,1223,1153,1112,1031,879,747,769,730 \mathrm{~cm}^{-1} ; \quad{ }^{1} \mathrm{H}$ NMR $(500 \mathrm{MHz}$, 
$\mathrm{CDCl}_{3}$ ) 7.21-7.11 (comp, 3H), 7.11-7.04 (m, 1H), 3.50-3.41 (m, 1H), 3.23 (ddd, $J=15.8$, 11.6, $5.8 \mathrm{~Hz}, 1 \mathrm{H}), 3.14(\mathrm{~d}, J=11.7 \mathrm{~Hz}, 1 \mathrm{H}), 3.08-2.99(\mathrm{~m}, 1 \mathrm{H}), 2.84(\mathrm{dd}, J=14.0,3.2 \mathrm{~Hz}$, 1H), 2.79-2.65 (comp, 2H), 2.54 (app td, $J=11.6,3.0 \mathrm{~Hz}, 1 \mathrm{H}), 2.28$ (d, $J=11.7 \mathrm{~Hz}, 1 \mathrm{H})$, 2.02-1.87 (comp, 2H), 1.85-1.71 (comp, 2H), 1.62-1.52 (m, 1H), 1.52-1.29 (comp, 4H), $1.23-1.11(\mathrm{~m}, 1 \mathrm{H}) ;{ }^{13} \mathrm{C} \mathrm{NMR}\left(125 \mathrm{MHz}, \mathrm{CDCl}_{3}\right) \delta 213.4,136.9,134.2,128.8,126.3,126.0$, 124.5, 64.8, 62.9, 51.5, 49.5, 44.4, 33.5, 30.4, 29.7, 26.1, 21.7, 21.6; $\mathrm{m} / z$ (ESI-MS) 270.3 $[\mathrm{M}+\mathrm{H}]^{+}, 302.1[\mathrm{M}+\mathrm{H}+\mathrm{MeOH}]^{+}$.

\section{1-cis-3,3-dimethyl-1-phenyl-3,4,6,7-tetrahydro-1H-pyrido[2,1-a]isoquinolin-2(11bH)-o}

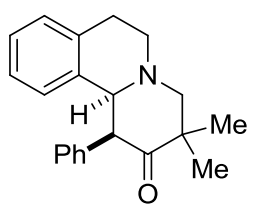

ne (cis-1g): Following the general procedure compound cis-1g was obtained from THIQ (95 $\mu \mathrm{L})$ and 2,2-dimethyl-3-oxo-4-phenylbutyraldehyde $(95 \mathrm{mg})$ as a yellow solid in $35 \%$ yield $(53 \mathrm{mg}),\left(\mathrm{R}_{\mathrm{f}}=0.64 \mathrm{in}\right.$ hexanes/EtOAc 80:20 v/v); mp: $114-117^{\circ} \mathrm{C}$; IR (KBr) 3061, 2950, 2755, 1698, 1597, 1494, 1466, 1401, 1379, 1353, 1291, 1247, 1113, 1047, 1031, 921, 897, 741, 719, 693, $628 \mathrm{~cm}^{-1} ; \quad{ }^{1} \mathrm{H}$ NMR (500 MHz, $\left.\mathrm{CDCl}_{3}\right)$ 7.60-7.52 (comp, $\left.2 \mathrm{H}\right), 7.12(\mathrm{~d}, J=7.6 \mathrm{~Hz}, 1 \mathrm{H})$, 7.09-6.98 (comp, 4H), 6.98-6.90 (comp, 2H), 4.34 (d, J = 4.4 Hz, 1H), 3.99 (d, J = $4.4 \mathrm{~Hz}$, $1 \mathrm{H}), 3.49-3.38(\mathrm{~m}, 1 \mathrm{H}), 3.26-3.17(\mathrm{~m}, 1 \mathrm{H}), 2.93(\mathrm{~d}, J=11.4 \mathrm{~Hz}, 1 \mathrm{H}), 2.90-2.83(\mathrm{~m}, 1 \mathrm{H})$, 2.63-2.55 (m, 1H), $2.51(\mathrm{~d}, J=11.4 \mathrm{~Hz}, 1 \mathrm{H}), 1.10(\mathrm{~s}, 3 \mathrm{H}), 1.01(\mathrm{~s}, 3 \mathrm{H}) ;{ }^{13} \mathrm{C} \mathrm{NMR}(125$ $\left.\mathrm{MHz}, \mathrm{CDCl}_{3}\right) \delta 213.1,136.0,135.3,134.7,129.8,128.6,127.6,126.1(9), 126.1(6), 125.9$, 124.6, 68.9, 65.2, 57.8, 52.4, 46.5, 29.7, 27.5, 23.4; $\mathrm{m} / \mathrm{z}(\mathrm{ESI}-\mathrm{MS}) 306.2[\mathrm{M}+\mathrm{H}]^{+}$.

\section{1-trans-3,3-dimethyl-1-phenyl-3,4,6,7-tetrahydro-1H-pyrido[2,1-a]isoquinolin-2(11bH)}

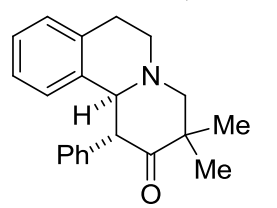
-one (trans-1g): Following the general procedure compound trans-1g was obtained from THIQ (95 $\mu \mathrm{L})$ and 2,2-dimethyl-3-oxo-4-phenylbutyraldehyde $(95 \mathrm{mg})$ as a yellow solid in $18 \%$ yield $(27 \mathrm{mg}),\left(\mathrm{R}_{\mathrm{f}}=0.54 \mathrm{in}\right.$ hexanes/EtOAc 80:20 v/v); mp: $98-100{ }^{\circ} \mathrm{C} ; \quad \mathrm{IR}(\mathrm{KBr}) 3059,3026,2829$, 1697, 1601, 1490, 1452, 1374, 1352, 1144, 1063, 946, 761, 726, $701 \mathrm{~cm}^{-1} ;{ }^{1} \mathrm{H}$ NMR (500 $\mathrm{MHz}, \mathrm{CDCl}_{3}$ ) 7.43-7.35 (comp, 2H), 7.35-7.29 (m, 1H), 7.25-7.20 (comp, 2H), 7.14-7.04 (comp, 2H), 6.84-6.77 (m, 1H), $6.39(\mathrm{~d}, J=8.1 \mathrm{~Hz}, 1 \mathrm{H}), 4.33(\mathrm{~d}, J=8.7 \mathrm{~Hz}, 1 \mathrm{H}), 3.86(\mathrm{~d}, J$ $=8.7 \mathrm{~Hz}, 1 \mathrm{H}), 3.28-3.13(\mathrm{comp}, 2 \mathrm{H}), 3.01(\mathrm{~d}, J=12.2 \mathrm{~Hz}, 1 \mathrm{H}), 2.97-2.86($ comp, 2H), 2.79-2.68 (m, 1H), $1.33(\mathrm{~s}, 3 \mathrm{H}), 1.14(\mathrm{~s}, 3 \mathrm{H}) ;{ }^{13} \mathrm{C} \mathrm{NMR}\left(125 \mathrm{MHz}, \mathrm{CDCl}_{3}\right) \delta 213.0,138.8$, 135.0, 130.3, 128.8, 128.6, 127.1, 127.0, 126.3, 125.3, 66.4, 65.4, 60.5, 51.6, 46.0, 29.4, 26.3, 23.5; $\mathrm{m} / \mathrm{z}(\mathrm{ESI}-\mathrm{MS}) 306.2[\mathrm{M}+\mathrm{H}]^{+}$.

11-trans-3-ethyl-3,4,6,7-tetrahydro-1H-pyrido[2,1-a]isoquinolin-2(11bH)-one

(1h):

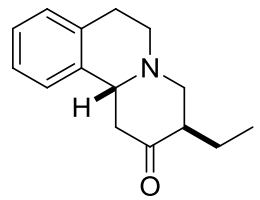
Following the general procedure compound $\mathbf{1 h}$ was obtained from THIQ (95 $\mu \mathrm{L})$ and 2-ethyl-3-oxobutyraldehyde $(57 \mathrm{mg}$ ) in $40 \%$ yield (46 mg, 6:1 mixture of two diastereomers). The major diastereomer was isolated as a yellow solid $\left(\mathrm{R}_{\mathrm{f}}=0.32\right.$ in hexanes/EtOAc 80:20 v/v); mp: $96-98^{\circ} \mathrm{C} ; \quad \mathrm{IR}$

(KBr) 3026, 2956, 2873, 2792, 1697, 1493, 1452, 1431, 1406, 1366, 1288, 1231, 1147, 1109, 1031, 889, 752, 735, $722 \mathrm{~cm}^{-1} ;{ }^{1} \mathrm{H}$ NMR (500 MHz, $\left.\mathrm{CDCl}_{3}\right)$ 7.20-7.11 (comp, 3H), 7.10-7.05 (m, 1H), 3.63-3.56 (m, 1H), $3.33(\mathrm{dd}, J=11.6,6.2 \mathrm{~Hz}, 1 \mathrm{H}), 3.25-3.12$ (comp, 2H), $2.94(\mathrm{dd}, J=13.7,3.1 \mathrm{~Hz}, 1 \mathrm{H}), 2.86-2.79(\mathrm{~m}, 1 \mathrm{H}), 2.68-2.50$ (comp, 3H), 2.39 (app t, $J=$ $11.6 \mathrm{~Hz}, 1 \mathrm{H}), 1.95-1.83(\mathrm{~m}, 1 \mathrm{H}), 1.32-1.21(\mathrm{~m}, 1 \mathrm{H}), 0.96(\mathrm{t}, J=7.5 \mathrm{~Hz}, 3 \mathrm{H}) ;{ }^{13} \mathrm{C}$ NMR 
$\left(125 \mathrm{MHz}, \mathrm{CDCl}_{3}\right) \delta 209.5,136.5,133.8,128.9,126.6,126.1,124.8,62.6,60.6,50.9,50.4$, 47.1, 29.6, 19.2, 11.6; $\mathrm{m} / \mathrm{z}\left(\right.$ ESI-MS) $262.0[\mathrm{M}+\mathrm{H}+\mathrm{MeOH}]^{+}$.

\section{1-trans-3-ethyl-9,10-dimethoxy-3,4,6,7-tetrahydro-1H-pyrido[2,1-a]isoquinolin-2(11bH}

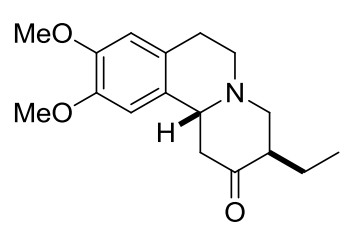

)-one (1i): Following the general procedure compound $\mathbf{1 i}$ was obtained from 6,7-dimethoxy-1,2,3,4-tetrahydroisoquinoline (145 mg) and 2-ethyl-3-oxobutyraldehyde $(57 \mathrm{mg})$ in $51 \%$ yield $(74 \mathrm{mg}, 6: 1$ mixture of two diastereomers). The major diastereomer was isolated as a yellow solid $\left(\mathrm{R}_{\mathrm{f}}=0.25\right.$ in hexanes/EtOAc 60:40 v/v); mp: 104-106 ${ }^{\circ} \mathrm{C}$; $\quad$ IR (KBr) 2937, 2835, 2795, 1705, 1609, 1519, 1467, 1410, 1370, 1331, 1289, $1262,1232,1158,1146,1105,1007,861,771 \mathrm{~cm}^{-1}$; ${ }^{1} \mathrm{H}$ NMR $\left(500 \mathrm{MHz}, \mathrm{CDCl}_{3}\right) 6.60(\mathrm{~s}$, $1 \mathrm{H}), 6.53(\mathrm{~s}, 1 \mathrm{H}), 3.83(\mathrm{~s}, 3 \mathrm{H}), 3.80(\mathrm{~s}, 3 \mathrm{H}), 3.53(\mathrm{app} \mathrm{d}, J=11.8 \mathrm{~Hz}, 1 \mathrm{H}), 3.32(\mathrm{dd}, J=11.6$, $6.2 \mathrm{~Hz}, 1 \mathrm{H}), 3.19-3.05$ (comp, 2H), 2.87 (dd, $J=13.6,2.8 \mathrm{~Hz}, 1 \mathrm{H}), 2.77-2.68$ (m, 1H), 2.67-2.49 (comp, 3H), 2.38 (app t, $J=11.6 \mathrm{~Hz}, 1 \mathrm{H}), 1.93-1.81(\mathrm{~m}, 1 \mathrm{H}), 1.29-1.17$ (m, 1H), $0.94(\mathrm{t}, J=7.5 \mathrm{~Hz}, 3 \mathrm{H}) ;{ }^{13} \mathrm{C} \mathrm{NMR}\left(125 \mathrm{MHz}, \mathrm{CDCl}_{3}\right) \delta 209.5,147.8,147.4,128.2,125.9$, $111.4,107.7,62.3,60.5,55.9,55.8,50.8,50.4,47.3,29.1,19.2,11.6 ; \quad \mathrm{m} / z$ (ESI-MS) 322.1 $[\mathrm{M}+\mathrm{H}+\mathrm{MeOH}]^{+}$.

12-trans-3-ethyl-3,4,6,7-tetrahydro-1H-[1,3]dioxolo[4,5-g]pyrido[2,1-a]isoquinolin-2(12b

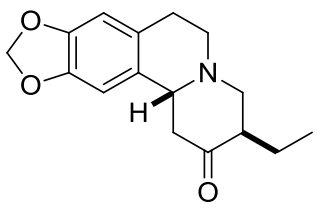

H)-one (1j): Following the general procedure compound $\mathbf{1 j}$ was obtained from 6,7-methylenedioxy-1,2,3,4-tetrahydroisoquinoline (133 $\mathrm{mg}$ ) and 2-ethyl-3-oxobutyraldehyde (57 mg) in $44 \%$ yield $(60 \mathrm{mg}, 7: 1$ mixture of two diastereomers). The major diastereomer was isolated as a yellow solid $\left(\mathrm{R}_{\mathrm{f}}=0.51\right.$ in hexanes/EtOAc 60:40 v/v); mp: $143-145^{\circ} \mathrm{C} ; \quad \mathrm{IR}(\mathrm{KBr}) 3046$, 2965, 2926, 2836, 2755, 1709, 1489, 1382, 1347, 1285, 1235, 1142, 1030, 921, 899, 869, 795, $753 \mathrm{~cm}^{-1} ;{ }^{1} \mathrm{H}$ NMR $\left(500 \mathrm{MHz}, \mathrm{CDCl}_{3}\right) 6.57(\mathrm{~s}, 1 \mathrm{H}), 6.52(\mathrm{~s}, 1 \mathrm{H}), 5.89(\mathrm{~s}, 2 \mathrm{H}), 3.52-3.45$ (m, 1H), 3.30 (dd, $J=11.5,6.2 \mathrm{~Hz}, 1 \mathrm{H}), 3.16-3.03$ (comp, 2H), $2.81(\mathrm{dd}, J=13.7,3.0 \mathrm{~Hz}$, $1 \mathrm{H}), 2.74-2.66(\mathrm{~m}, 1 \mathrm{H}), 2.65-2.53$ (comp, 2H), 2.49 (app t, $J=13.0 \mathrm{~Hz}, 1 \mathrm{H}), 2.36$ (app t, $J=$ $11.5 \mathrm{~Hz}, 1 \mathrm{H}), 1.93-1.82(\mathrm{~m}, 1 \mathrm{H}), 1.30-1.18(\mathrm{~m}, 1 \mathrm{H}), 0.94(\mathrm{t}, J=7.5 \mathrm{~Hz}, 3 \mathrm{H}) ;{ }^{13} \mathrm{C} \mathrm{NMR}$ $\left(125 \mathrm{MHz}, \mathrm{CDCl}_{3}\right) \delta 209.3,146.3,146.2,129.5,127.1,108.5,104.9,100.8,62.6,60.5,50.8$, 50.4, 47.4, 29.7, 19.2, 11.6; $\mathrm{m} / z$ (ESI-MS) $306.1[\mathrm{M}+\mathrm{H}+\mathrm{MeOH}]^{+}$.

\section{2-trans-3-ethyl-12-methyl-1,3,4,6,7,12b-hexahydroindolo[2,3-a]quinolizin-2(12H)-one}

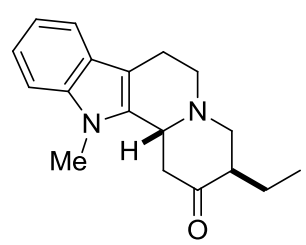

(1k): Following the general procedure compound $1 \mathbf{k}$ was obtained from 9-methyltryptoline $(140 \mathrm{mg})$ and 2-ethyl-3-oxobutyraldehyde $(57 \mathrm{mg})$ in $45 \%$ yield (64 mg, 8:1 mixture of two diastereomers). The major diastereomer was isolated as a yellow solid $\left(\mathrm{R}_{\mathrm{f}}=0.51\right.$ in hexanes/EtOAc 60:40 v/v); $\mathrm{mp}: 177-178{ }^{\circ} \mathrm{C}$; $\quad \mathrm{IR}(\mathrm{KBr}) 3046,2958,2923,2876,2835$, 2803, 1701, 1471, 1425, 1380, 1363, 1316, 1299, 1283, 1184, 1150, 1087, 1000, 736, 696 $\mathrm{cm}^{-1} ;{ }^{1} \mathrm{H}$ NMR $\left(500 \mathrm{MHz}, \mathrm{CDCl}_{3}\right) 7.53(\mathrm{~d}, J=7.8 \mathrm{~Hz}, 1 \mathrm{H}), 7.28(\mathrm{~d}, J=8.2 \mathrm{~Hz}, 1 \mathrm{H})$, 7.25-7.20 (m, 1H), 7.15-7.10 (m, 1H), $4.10(\mathrm{dd}, J=11.4,2.8 \mathrm{~Hz}, 1 \mathrm{H}), 3.64(\mathrm{~s}, 3 \mathrm{H}), 3.48$ (dd, $J=12.9,6.1 \mathrm{~Hz}, 1 \mathrm{H}), 3.38-3.30(\mathrm{~m}, 1 \mathrm{H}), 3.07-2.90$ (comp, 3H), 2.85 (app t, $J=12.9 \mathrm{~Hz}$, $1 \mathrm{H}), 2.77$ (dd, $J=13.6,3.2 \mathrm{~Hz}, 1 \mathrm{H}), 2.73-2.58$ (comp, 2H), 1.97-1.86 (m, 1H), 1.34-1.21 (m, $1 \mathrm{H}), 1.00(\mathrm{t}, J=7.5 \mathrm{~Hz}, 3 \mathrm{H}) ;{ }^{13} \mathrm{C} \mathrm{NMR}\left(125 \mathrm{MHz}, \mathrm{CDCl}_{3}\right) \delta 209.0,137.6,135.0,126.3$, 
121.5, 119.1, 118.2, 108.9, 107.7, 60.4, 57.7, 49.2, 47.3, 45.2, 30.3, 22.3, 19.2, 11.6; $\mathrm{m} / \mathrm{z}$ (ESI-MS) $315.1[\mathrm{M}+\mathrm{H}+\mathrm{MeOH}]^{+}$.

\section{1-trans-3-butyl-9,10-dimethoxy-3,4,6,7-tetrahydro-1H-pyrido[2,1-a]isoquinolin-2(11b}

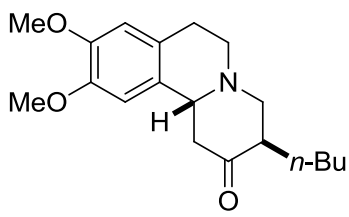

H)-one (11): Following the general procedure compound $\mathbf{1 l}$ was obtained from 6,7-dimethoxy-1,2,3,4-tetrahydroisoquinoline (145 mg) and 2-n-butyl-3-oxobutyraldehyde (71 mg) in 56\% yield (89 $\mathrm{mg}, 13: 1$ mixture of two diastereomers). The major diastereomer was isolated as a yellow solid $\left(\mathrm{R}_{\mathrm{f}}=0.4\right.$ in hexanes/EtOAc 60:40 v/v); mp: $110-111^{\circ} \mathrm{C}$; $\quad \operatorname{IR}(\mathrm{KBr}) 2960,2854,2760,1701,1610,1514,1466,1408,1363,1290,1248$, 1160, 1108, 1033, 1009, 872, $768 \mathrm{~cm}^{-1}$; ${ }^{1} \mathrm{H}$ NMR (500 MHz, $\left.\mathrm{CDCl}_{3}\right) 6.59$ (s, 1H), 6.52 (s, $1 \mathrm{H}), 3.82(\mathrm{~s}, 3 \mathrm{H}), 3.80(\mathrm{~s}, 3 \mathrm{H}), 3.48(\mathrm{dd}, J=12.0,2.2 \mathrm{~Hz}, 1 \mathrm{H}), 3.29(\mathrm{dd}, J=12.0,6.3 \mathrm{~Hz}$, 1H), 3.17-3.03 (comp, 2H), 2.86 (dd, $J=13.6,3.0 \mathrm{~Hz}, 1 \mathrm{H}$ ), 2.76-2.45 (comp, 4H), 2.35 (app $\mathrm{t}, J=11.7 \mathrm{~Hz}, 1 \mathrm{H}), 1.91-1.80(\mathrm{~m}, 1 \mathrm{H}), 1.43-1.24$ (comp, 4H), 1.21-1.11 (m, 1H), 0.95-0.86 $(\mathrm{m}, 3 \mathrm{H}) ;{ }^{13} \mathrm{C}$ NMR $\left(125 \mathrm{MHz}, \mathrm{CDCl}_{3}\right) \delta 209.8,147.7,147.3,128.4,126.0,111.3,107.7$, $62.3,61.0,55.8,55.7,50.4,49.4,47.4,29.3,29.2,25.7,22.7,13.9 ; \quad \mathrm{m} / z$ (ESI-MS) 318.3 [M $+\mathrm{H}]^{+}$.

11-trans-3-isobutyl-3,4,6,7-tetrahydro-1H-pyrido[2,1-a]isoquinolin-2(11bH)-one (1m):<smiles>CC(C)CC1CN2CCc3ccccc3CC2CC1=O</smiles>

Following the general procedure compound $\mathbf{1 m}$ was obtained from THIQ $(95 \mu \mathrm{L})$ and 2-i-butyl-3-oxobutyraldehyde $(71 \mathrm{mg})$ as a yellow oil in $34 \%$ yield (44 mg, 3:1 mixture of two diastereomers) $\left(\mathrm{R}_{\mathrm{f}}=0.41\right.$ in hexanes/EtOAc 90:10 v/v); IR (KBr) 3061, 3019, 2954, 2920, 2866, 2806, 2757, 1712, 1630, 1585, 1550, 1490, 1466, 1367, 1297, 1247, 1220, 1144, 1109, 742 $\mathrm{cm}^{-1}$; ${ }^{1} \mathrm{H}$ NMR $\left(500 \mathrm{MHz}, \mathrm{CDCl}_{3}\right.$ ) (Note: due to overlapping peaks, integration values of the diastereomers are reported together) 7.21-7.11 (comp, 3.14H), 7.11-7.05 (comp, 1.14H), 3.64-3.50 (comp, 1.21H), 3.30 (dd, $J=11.5,6.3 \mathrm{~Hz}, 1.00 \mathrm{H}), 3.26-3.11$ (comp, 2.09H), 3.07-2.70 (comp, 4.18H), 2.69-2.50 (comp, 2.63H), 2.36 (app t, $J=11.7 \mathrm{~Hz}, 1.04 \mathrm{H})$, 1.86-1.74 (comp, 1.29H), 1.73-1.60 (comp, 1.11H), 1.59-1.48 (m, 0.59H), 1.09-0.99 (m, $1.08 \mathrm{H}), 0.99-0.82$ (comp, 6.83H); ${ }^{13} \mathrm{C}$ NMR of the diastereomers $\left(125 \mathrm{MHz}, \mathrm{CDCl}_{3}\right) \delta$ 209.7, 136.5, 133.8, 128.9(5), 128.8(9), 126.6, 126.5, 126.1, 124.8, 124.7, 62.6, 61.4, 50.4, 47.4, 47.2, 35.0, 29.6, 25.7, 25.3, 23.2, 22.6, 22.3, 22.0; $\mathrm{m} / \mathrm{z}$ (ESI-MS) $290.1[\mathrm{M}+\mathrm{H}+$ $\mathrm{MeOH}]^{+}$.

11-trans-3-benzyl-9,10-dimethoxy-3,4,6,7-tetrahydro-1H-pyrido[2,1-a]isoquinolin-2(11b

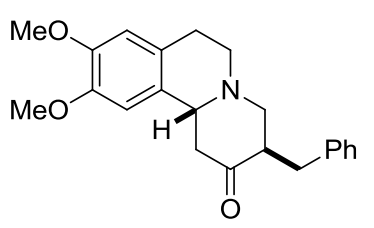

H)-one (10): Following the general procedure compound 1o was obtained from 6,7-dimethoxy-1,2,3,4-tetrahydroisoquinoline (145 mg) and 2-benzyl-3-oxobutyraldehyde (88 $\mathrm{mg}$ ) in $60 \%$ yield $(105 \mathrm{mg}$, 6.5:1 mixture of two diastereomers). The major diastereomer was isolated as a yellow solid $\left(\mathrm{R}_{\mathrm{f}}=0.39\right.$ in hexanes/EtOAc 60:40 v/v); mp: $114-117^{\circ} \mathrm{C}$; IR (KBr) 3009, 2930, 2834, 2809, 2761, 1701, 1611, 1514, 1465, 1453, $1366,1328,1256,1228,1151,1112,1098,1028,1010,911,857,766,735,704 \mathrm{~cm}^{-1} ; \quad{ }^{1} \mathrm{H}$ NMR (500 MHz, $\mathrm{CDCl}_{3}$ ) 7.31-7.25 (comp, 2H), 7.23-7.15 (comp, 3H), 6.59 (s, 1H), 6.54 (s, $1 \mathrm{H}), 3.82(5)(\mathrm{s}, 3 \mathrm{H}), 3.81(6)(\mathrm{s}, 3 \mathrm{H}), 3.56-3.49(\mathrm{~m}, 1 \mathrm{H}), 3.33(\mathrm{dd}, J=14.2,4.4 \mathrm{~Hz}, 1 \mathrm{H}), 3.13$ 
(dd, $J=11.6,6.2 \mathrm{~Hz}, 1 \mathrm{H}), 3.08-2.91(\mathrm{comp}, 4 \mathrm{H}), 2.73-2.61(\mathrm{~m}, 1 \mathrm{H}), 2.61-2.48(\mathrm{comp}, 2 \mathrm{H})$, 2.47-2.34 (comp, 2H); ${ }^{13} \mathrm{C}$ NMR (125 MHz, $\left.\mathrm{CDCl}_{3}\right) \delta 208.8,147.6,147.3,139.3,128.8$, 128.3, 128.2, 126.0, 125.9, 111.3, 107.7, 62.2, 60.3, 55.8, 55.7, 50.9, 50.2, 47.3, 32.4, 29.1; $\mathrm{m} / z(\mathrm{ESI}-\mathrm{MS}) 384.0[\mathrm{M}+\mathrm{H}+\mathrm{MeOH}]^{+}$.

\section{2-trans-3-benzyl-12-methyl-1,3,4,6,7,12b-hexahydroindolo[2,3-a]quinolizin-2(12H)-one}

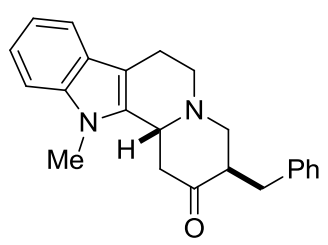

(1p): Following the general procedure compound 1p was obtained from 9-methyltryptoline (140 $\mathrm{mg}$ ) and 2-benzyl-3-oxobutyraldehyde (88 $\mathrm{mg}$ ) in $53 \%$ yield (91 $\mathrm{mg}, 8: 1 \mathrm{mixture}$ of two diastereomers). The major diastereomer was isolated as a yellow oil $\left(R_{\mathrm{f}}=0.47\right.$ in hexanes/EtOAc 70:30 v/v); IR (KBr) 3057, 3027, 2950, 2918, 2843, 1701, 1496, 1470, 1454, 1421, 1385, 1338, 1309, 1273, 1216, 1188, 1142, 1077, 1031, 1013, 910, 737, $700 \mathrm{~cm}^{-1} ; \quad{ }^{1} \mathrm{H}$ NMR $\left(500 \mathrm{MHz}, \mathrm{CDCl}_{3}\right)$ 7.54-7.50 (m, 1H), 7.37-7.31 (comp, 2H), 7.31-7.21 (comp, 5H), 7.16-7.11 (m, 1H), 4.12 (dd, $J=11.6,2.6 \mathrm{~Hz}, 1 \mathrm{H}), 3.65(\mathrm{~s}, 3 \mathrm{H})$, 3.41-3.33 (comp, 2H), 3.24 (ddd, $J=11.0,6.2,4.3 \mathrm{~Hz}, 1 \mathrm{H}), 3.06-2.98$ (m, 1H), 2.96-2.79 (comp, 5H), $2.74($ app t, $J=12.1 \mathrm{~Hz}, 1 \mathrm{H}), 2.46(\mathrm{dd}, J=14.3,8.8 \mathrm{~Hz}, 1 \mathrm{H}) ;{ }^{13} \mathrm{C} \mathrm{NMR}(125$ $\left.\mathrm{MHz}, \mathrm{CDCl}_{3}\right) \delta 208.2,139.4,137.6,134.8,128.9,128.5,126.3,126.2,121.6,119.2,118.2$, $108.9,107.8,60.1,57.8,49.4,47.3,45.2,32.4,30.4,22.2 ; \quad m / z(E S I-M S) 377.1[\mathrm{M}+\mathrm{H}+$ $\mathrm{MeOH}]^{+}$.

\section{1-trans-3-(4-(benzyloxy)butyl)-9,10-dimethoxy-3,4,6,7-tetrahydro-1H-pyrido[2,1-a]isoq}

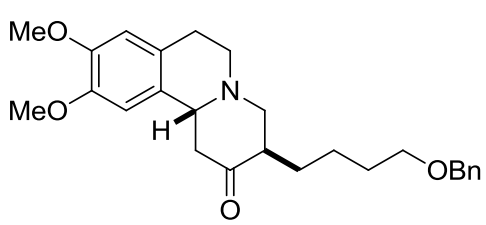
uinolin-2(11bH)-one (1q): Following the general procedure compound 1q was obtained from 6,7-dimethoxy-1,2,3,4tetrahydroisoquinoline (145 mg) and 2-(4-benzyloxy)butyl-3oxobutyraldehyde $(124 \mathrm{mg})$ in $57 \%$ yield $(121 \mathrm{mg}$, 5:1 mixture of two diiastereomers). The major diastereomer was isolated as a yellow solid $\left(\mathrm{R}_{\mathrm{f}}=0.23\right.$ in hexanes/EtOAc $\left.60: 40 \mathrm{v} / \mathrm{v}\right) ; \mathrm{mp}: 101-103{ }^{\circ} \mathrm{C}$; IR (KBr) 2992, 2940, 2849, 1702, 1609, 1517, 1463, 1367, 1328, 1291, 1262, 1210, 1156, 1141, 1100, 1011, 867, $734 \mathrm{~cm}^{-1}$; ${ }^{1} \mathrm{H}$ NMR (500 MHz, $\mathrm{CDCl}_{3}$ ) 7.37-7.30 (comp, 4H), 7.30-7.24 (m, 1H), $6.61(\mathrm{~s}, 1 \mathrm{H}), 6.54(\mathrm{~s}, 1 \mathrm{H}), 4.50(\mathrm{~s}, 2 \mathrm{H}), 3.85$ (s, 3H), 3.82 (s, 3H), 3.51-3.46 (comp, $3 \mathrm{H}), 3.31(\mathrm{dd}, J=11.4,6.2 \mathrm{~Hz}, 1 \mathrm{H}), 3.17-3.05$ (comp, 2H), 2.88 (dd, $J=13.6,2.4 \mathrm{~Hz}, 1 \mathrm{H})$, 2.77-2.63 (comp, 2H), 2.63-2.47 (comp, 2H), 2.38 (app t, $J=11.8 \mathrm{~Hz}, 1 \mathrm{H}), 1.95-1.83$ (m, 1H), 1.72-1.56 (comp, 2H), 1.50-1.36 (comp, 2H), $1.28-1.14(\mathrm{~m}, 1 \mathrm{H}) ;{ }^{13} \mathrm{C}$ NMR $(125$ $\left.\mathrm{MHz}, \mathrm{CDCl}_{3}\right) \delta 209.7,147.8,147.4,138.6,128.4,128.3,127.6,127.5,126.0,111.4,107.8$, 72.9, 70.1, 62.3, 61.0, 55.9, 55.8, 50.4, 49.4, 47.5, 29.9, 29.3, 25.9, 23.8; $\mathrm{m} / z$ (ESI-MS) $456.0\left({ }^{35} \mathrm{Cl} /{ }^{35} \mathrm{Cl}\right)[\mathrm{M}+\mathrm{H}+\mathrm{MeOH}]^{+}$. 
2D-NMR Analysis for Compound cis-1g, Selected Interactions:

GcosY

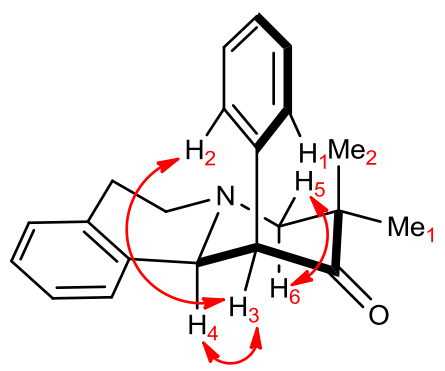

NOESY

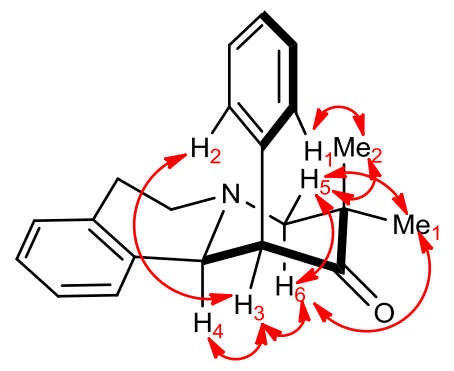

${ }^{1} \mathrm{H}$ NMR shifts

\begin{tabular}{|c|c|}
\hline Proton & Chemical Shift $(\mathrm{ppm})$ \\
\hline H1, H2 & $7.60-7.52$ \\
\hline H3 & 4.34 \\
\hline H4 & 3.99 \\
\hline H5 & 2.93 \\
\hline H6 & 2.51 \\
\hline Me1 & 1.10 \\
\hline Me2 & 1.01 \\
\hline
\end{tabular}




\section{References:}

(1) Matulenko, M. A.; Meyers, A. I. J. Org. Chem. 1996, 61, 573.

(2) Kikuchi, C.; Ando, T.; Watanabe, T.; Nagaso, H.; Okuno, M.; Hiranuma, T.; Koyama, M. J. Med. Chem. 2002, 45, 2197.

(3) Inukai, T.; Yoshizawa, R. J. Org. Chem. 1966, 32, 404.

(4) Lovchik, M. A.; Goeke, A.; Frater, G. J. Org. Chem. 2007, 72, 2427.

(5) Fujita, T.; Sakoda, K.; Ikeda, M.; Hattori, M.; Ichikawa, J. Synlett 2013, 24, 57.

(6) Frankowski, K. J.; Golden, J. E.; Zeng, Y.; Lei, Y.; Aubé, J. J. Am. Soc. Chem. 2008, 130, 6018.

(7) Sun, X.; Zhu, R.; Li, G.; Ma, X.; Gu, Z. J. Am. Chem. Soc. 2013, 135, 9318.

(8) Yao, Z.; Wei, X.; Wu, X.; Katz, J. L.; Kopajtic, T.; Greig, N. H.; Sun, H. Eur. J. Med. Chem. 2011, 46, 1841.

(9) a) Nakamura, K.; Miyai, T.; Nagar, A.; Oka, S.; Ohno, A. Bull. Chem. Soc, Jpn. 1989, 62, 1179;

b) Li, W.; Wang, J.; Hu, X.; Shen, K.; Wang, W.; Chu, Y.; Lin, L.; Liu, X.; Feng, X. J. Am. Chem. Soc. 2010, 132, 8532; c) Biju, A. T.; Padmanaban, M.; Wurz, N. E.; Glorius, F. Angew. Chem. Int. Ed. 2011, 50, 8412; d) Hashimoto, T.; Naganawa, Y.; Maruoka, K. J. Am. Chem. Soc. 2011, 133, 8834.

(10) Bastian, J. A.; Lash, T. D. Tetrahedron, 1988, 54, 6299.

(11) Martin, V. A.; Murray, D. H.; Pratt, N. E.; Zhao, Y.; Albizati, K. F. J. Am. Chem. Soc. 1990, 112,6965 .

(12) Riva, R.; Banfi, L.; Basso, A.; Zito, P.; Org. Biomol. Chem. 2011, 9, 2107.

(13) Hirai, K.; Ooi, H.; Esumi, T.; Iwabuchi, Y.; Hatakeyama, S. Org. Lett. 2003, 5, 857. 

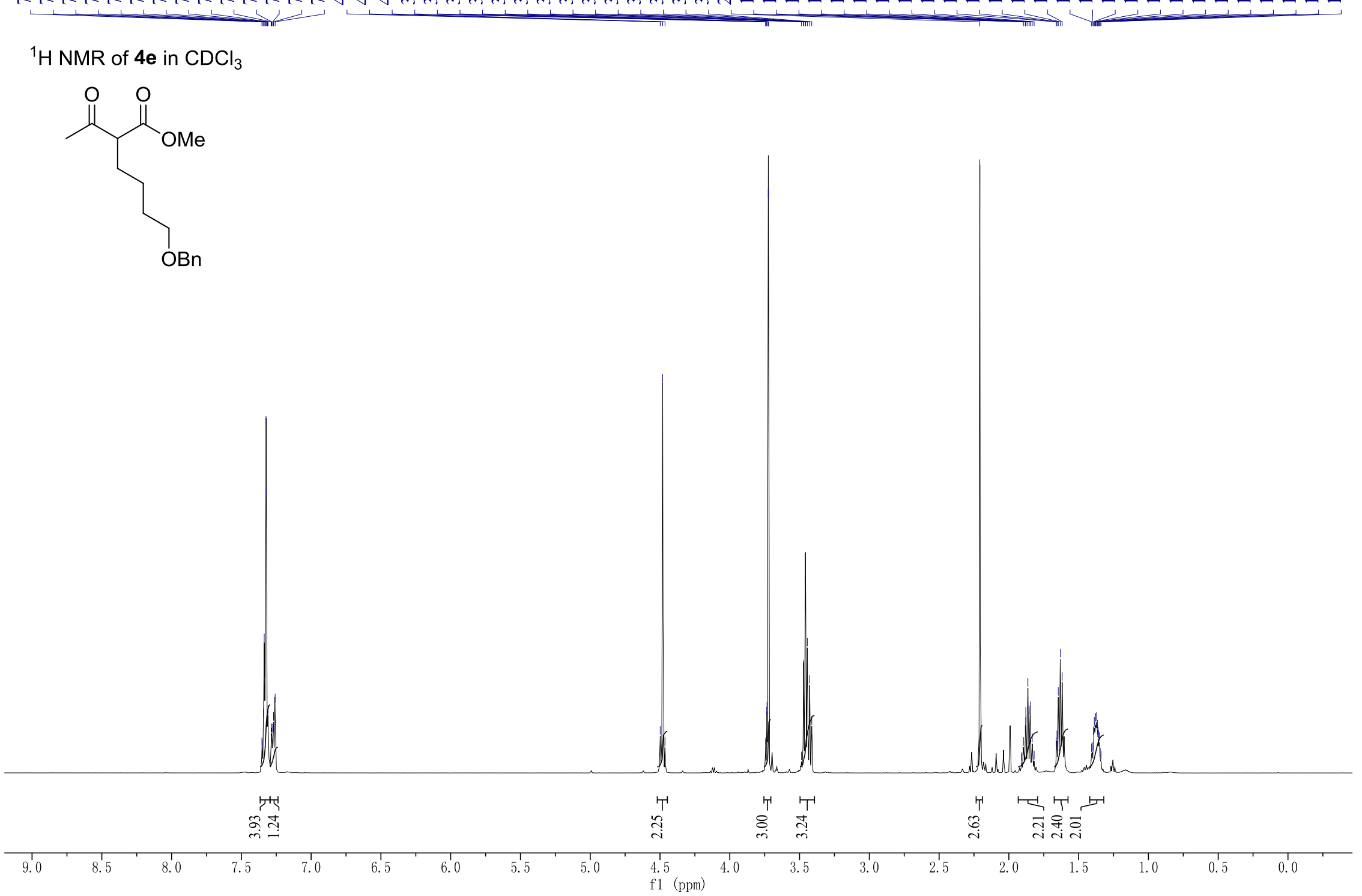

ㅎํํ.

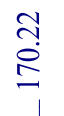

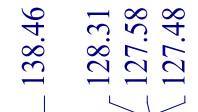

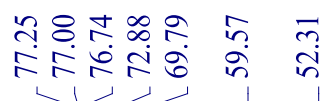

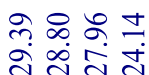

${ }^{13} \mathrm{C} \mathrm{NMR}$ of $4 \mathbf{e}$ in $\mathrm{CDCl}_{3}$
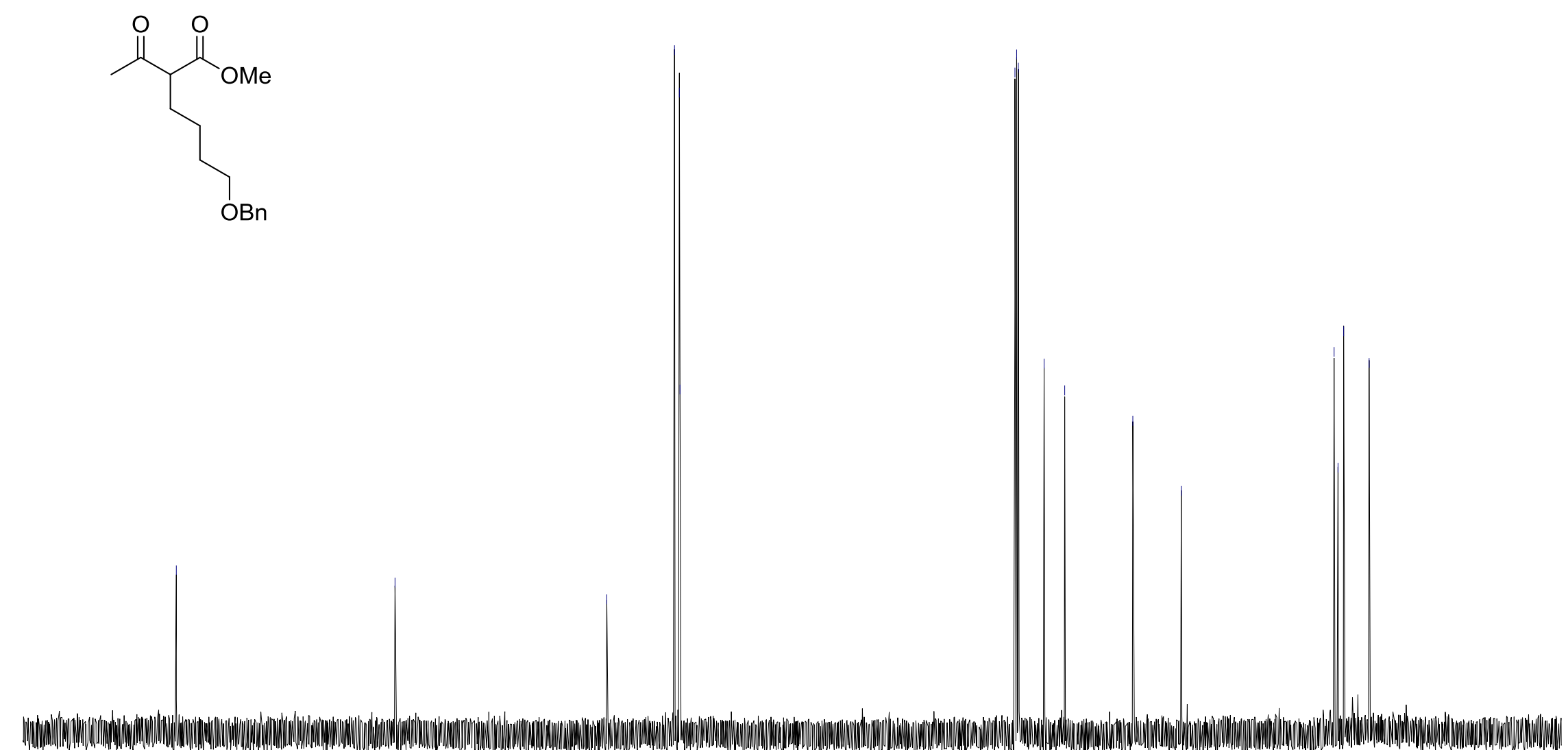

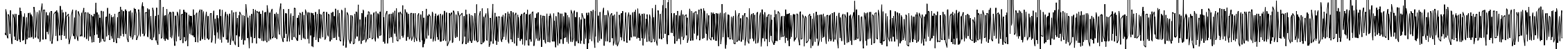

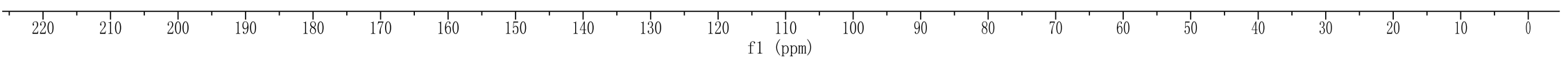




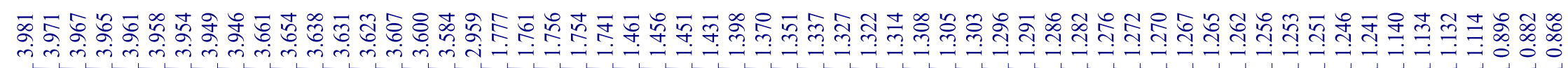
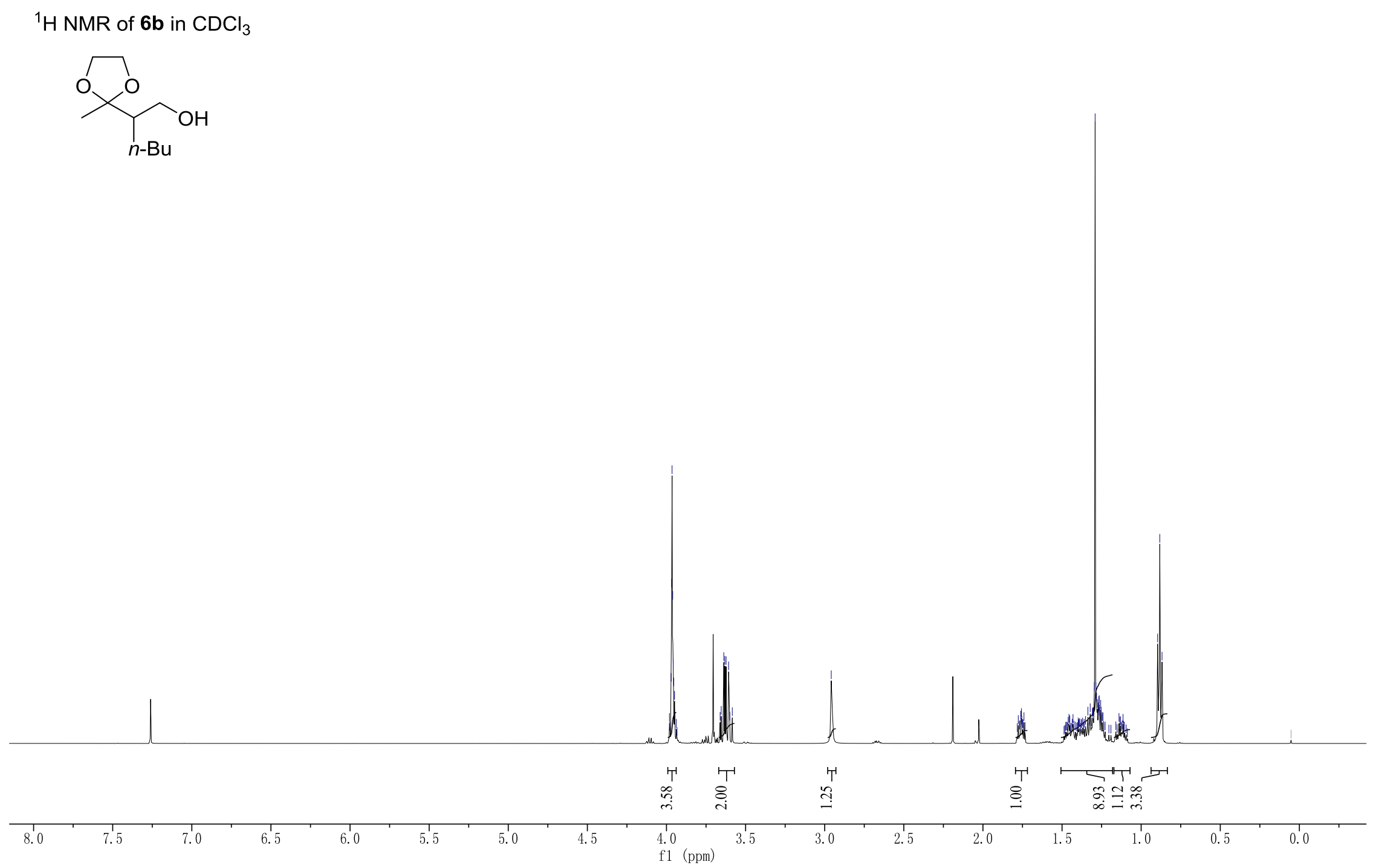


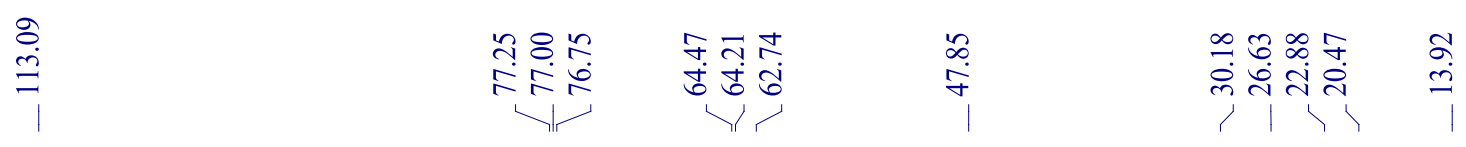

${ }^{13} \mathrm{C}$ NMR of $\mathbf{6 b}$ in $\mathrm{CDCl}_{3}$<smiles>CCCCC(CO)C1(C)OCCO1</smiles>

(1)

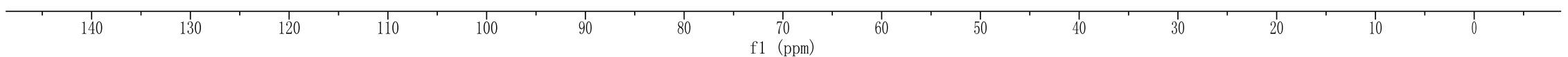




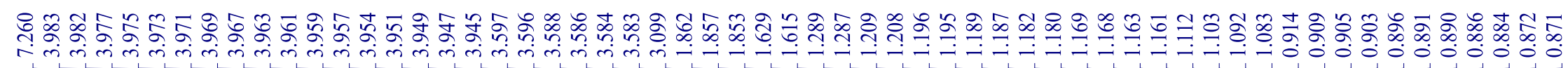

\section{${ }^{1} \mathrm{H}$ NMR of $6 \mathrm{c}$ in $\mathrm{CDCl}_{3}$}
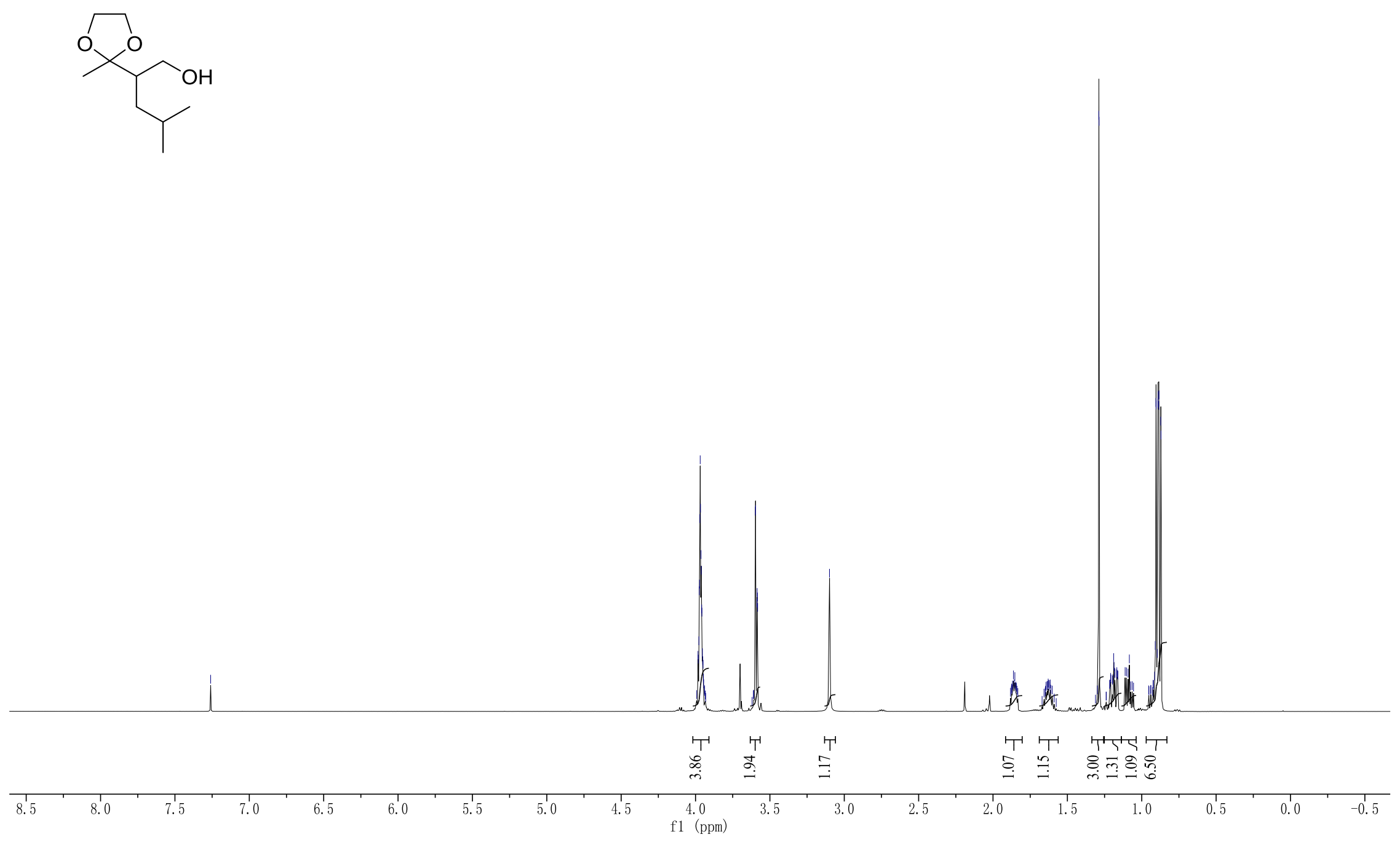


至

${ }^{13} \mathrm{C} \mathrm{NMR}$ of $\mathbf{6 c}$ in $\mathrm{CDCl}_{3}$

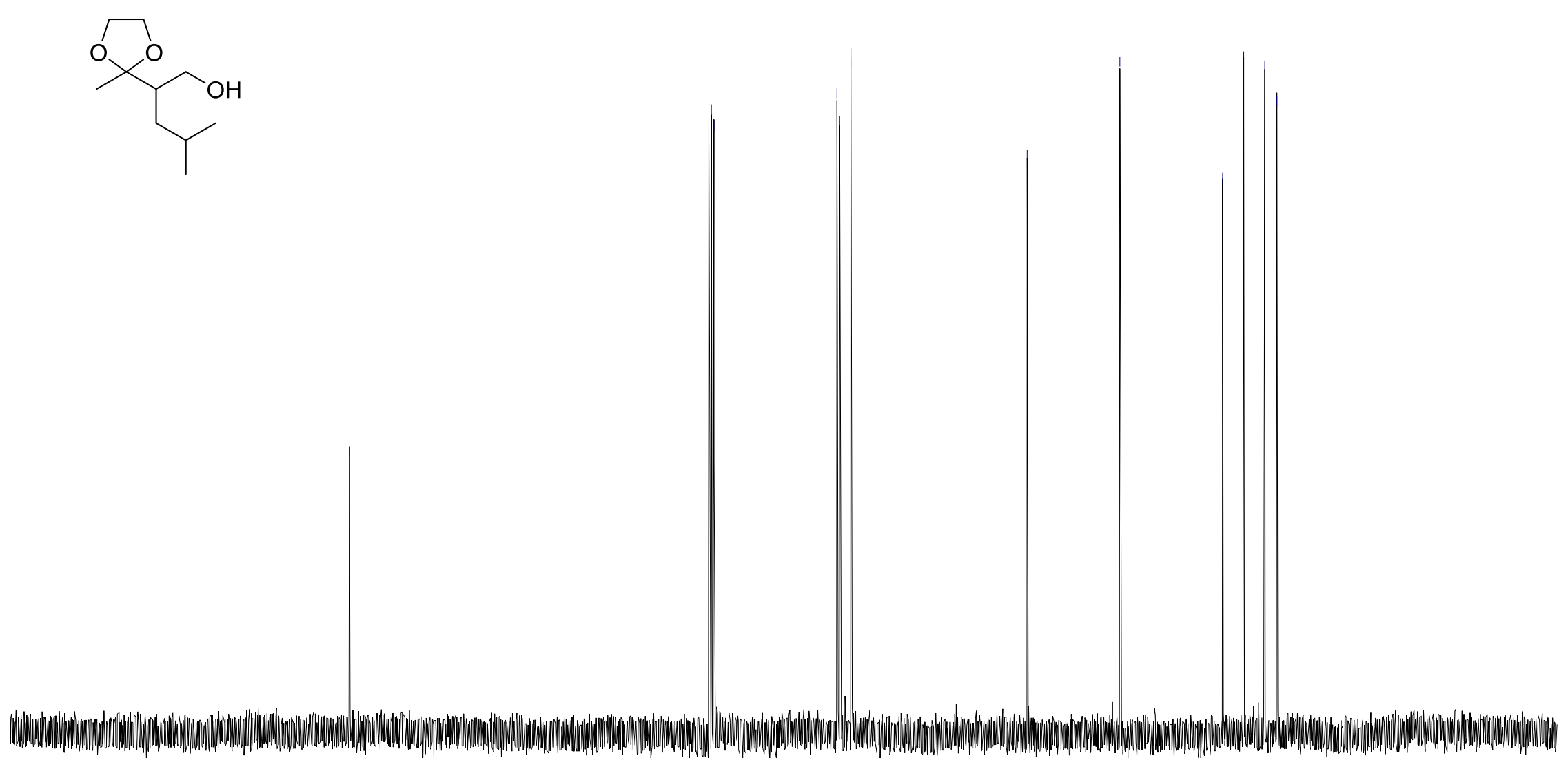

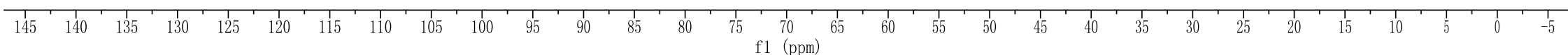




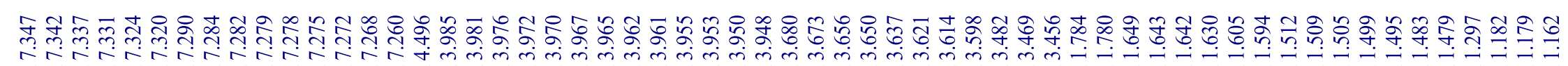

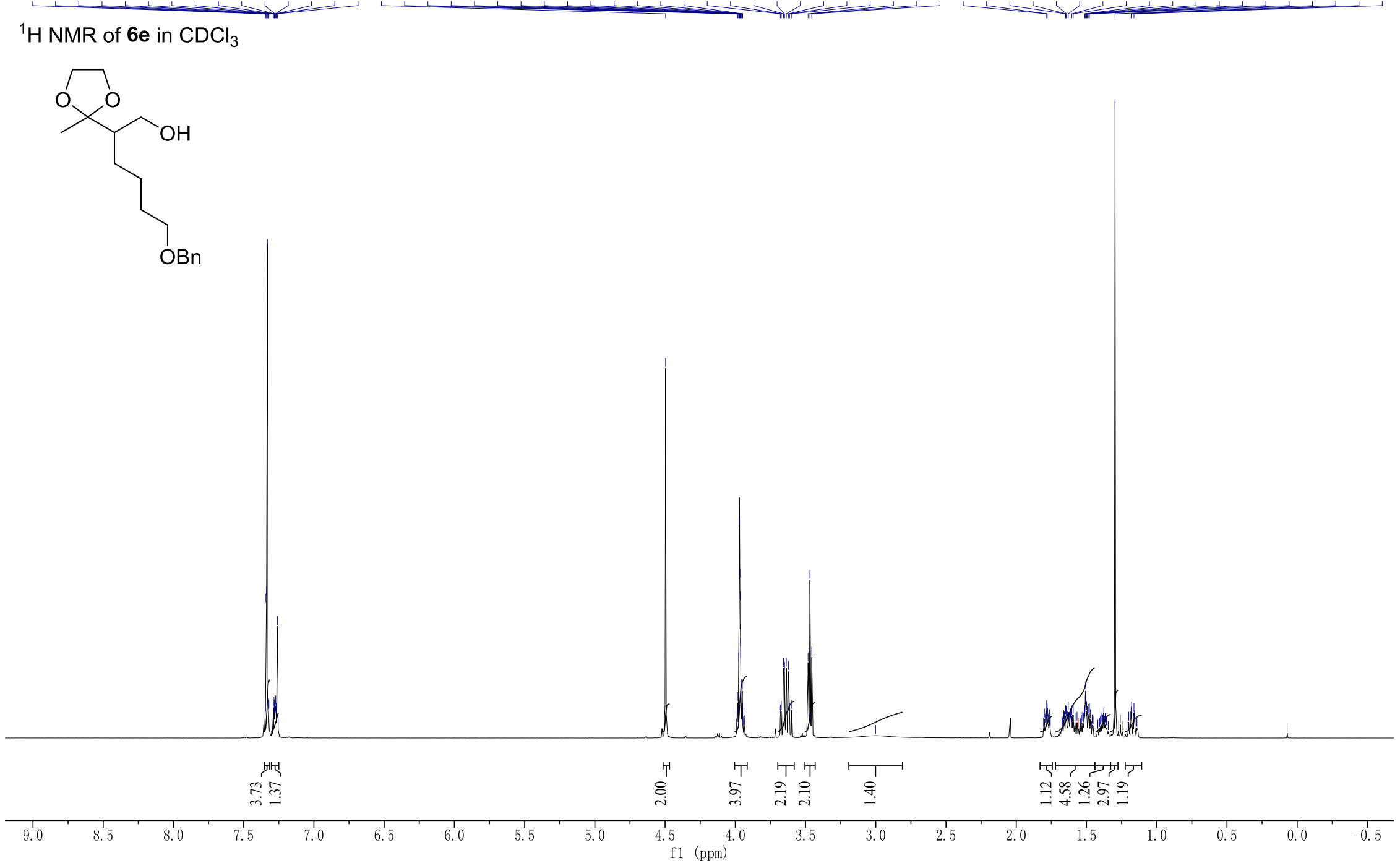




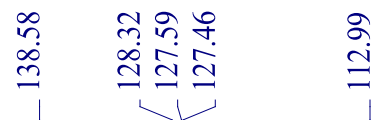

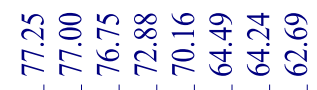
$\underset{\dot{\infty}}{\stackrel{\leftrightarrow}{+}}$
ำ

${ }^{13} \mathrm{C}$ NMR of $6 \mathbf{e}$ in $\mathrm{CDCl}_{3}$

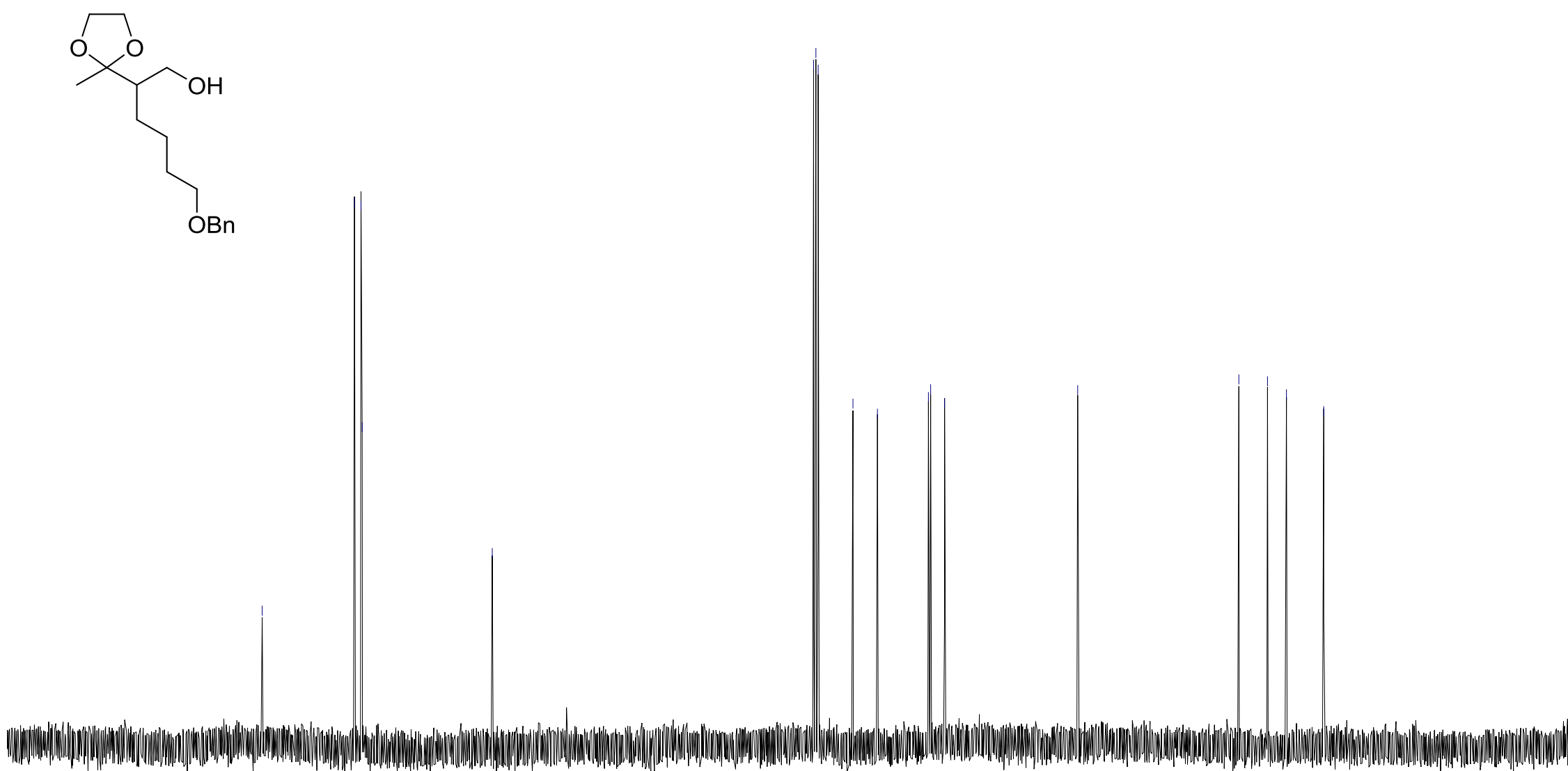

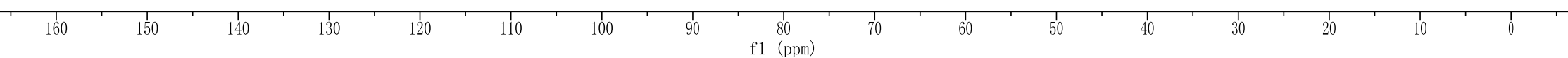


${ }^{1} \mathrm{H}$ NMR of $\mathbf{8 a}$ in $\mathrm{CDCl}_{3}$

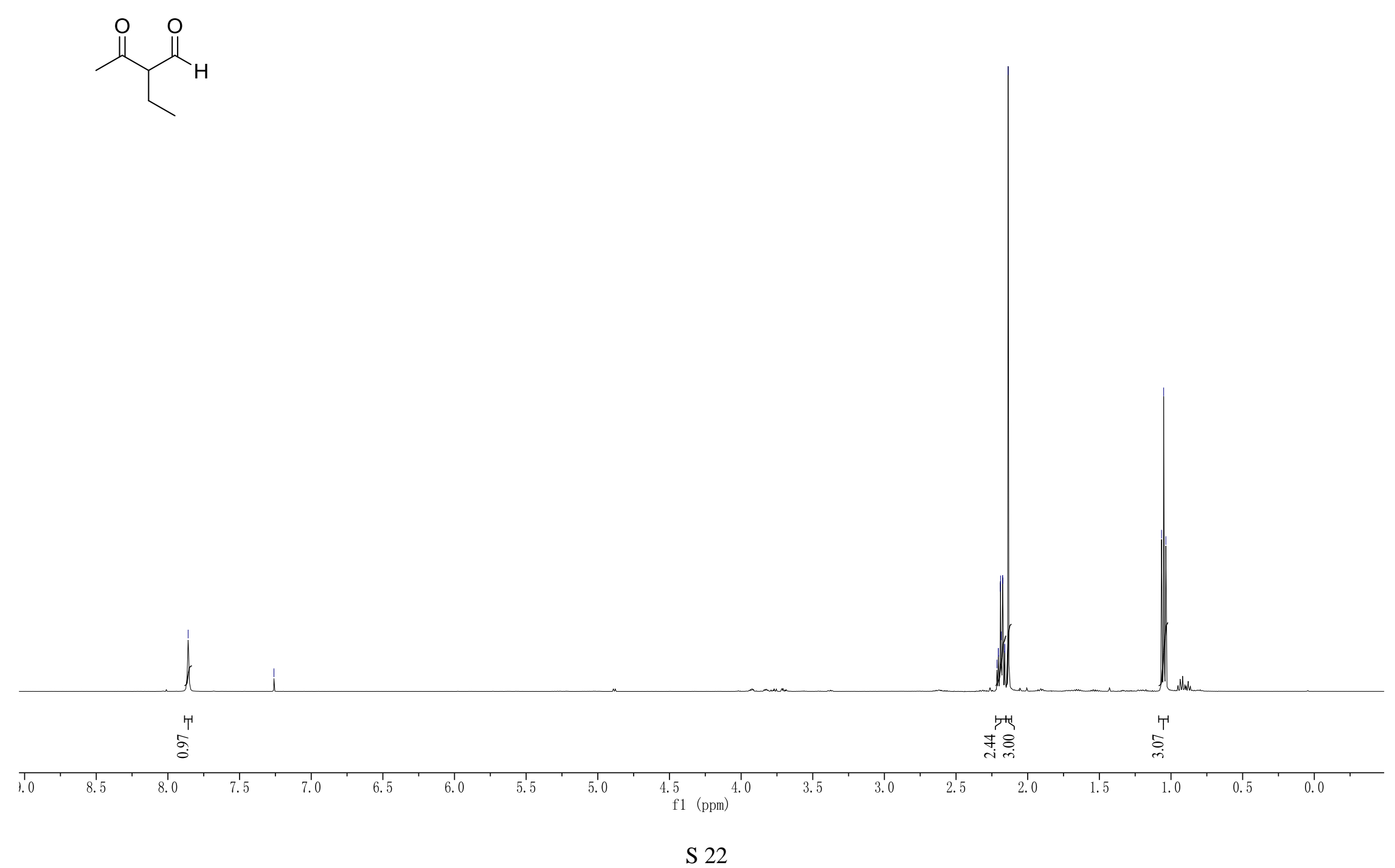




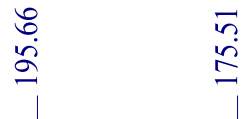
$\stackrel{?}{\stackrel{9}{\Xi}}$

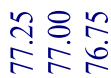
훟월

${ }^{13} \mathrm{C}$ NMR of $\mathbf{8} \mathbf{a}$ in $\mathrm{CDCl}_{3}$<smiles>CCC(C=O)C(C)=O</smiles>
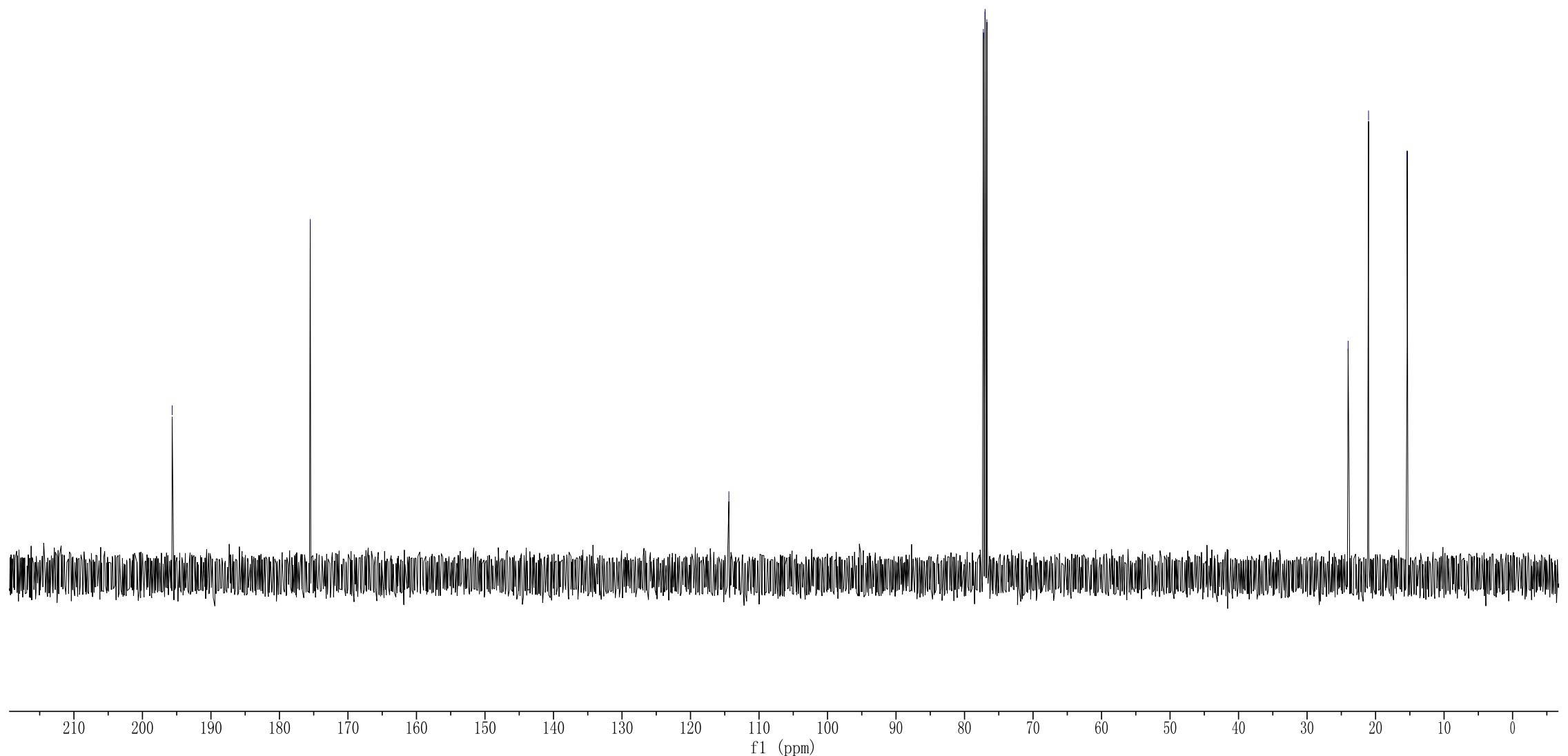
ণ্ণু

${ }^{1} \mathrm{H} \mathrm{NMR}$ of $\mathbf{8} \mathbf{b}$ in $\mathrm{CDCl}_{3}$

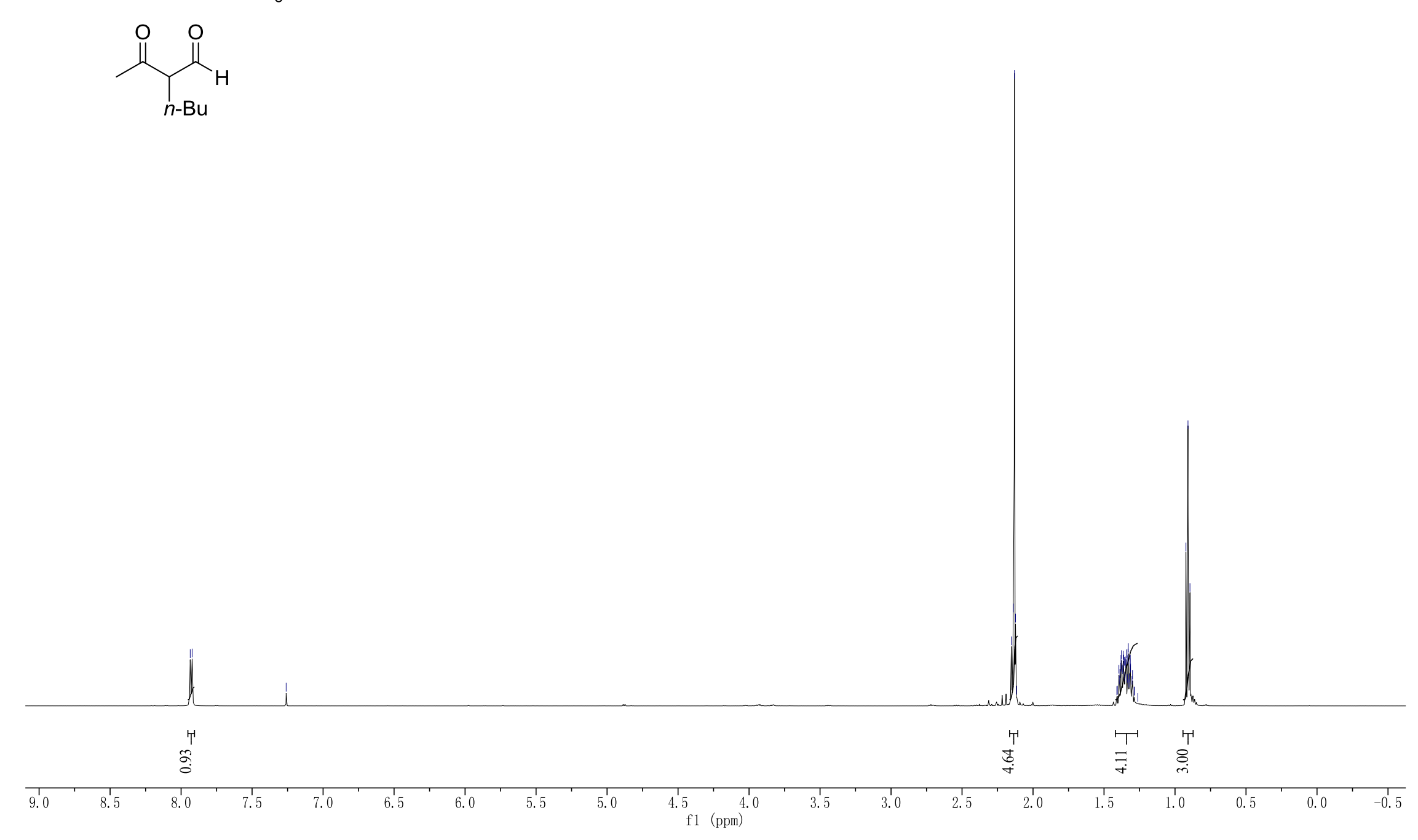

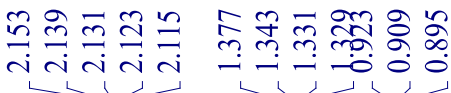




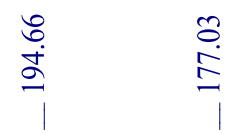
$\stackrel{\infty}{\Xi}$

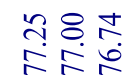

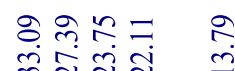

${ }^{13} \mathrm{C} \mathrm{NMR}$ of $\mathbf{8 b}$ in $\mathrm{CDCl}_{3}$
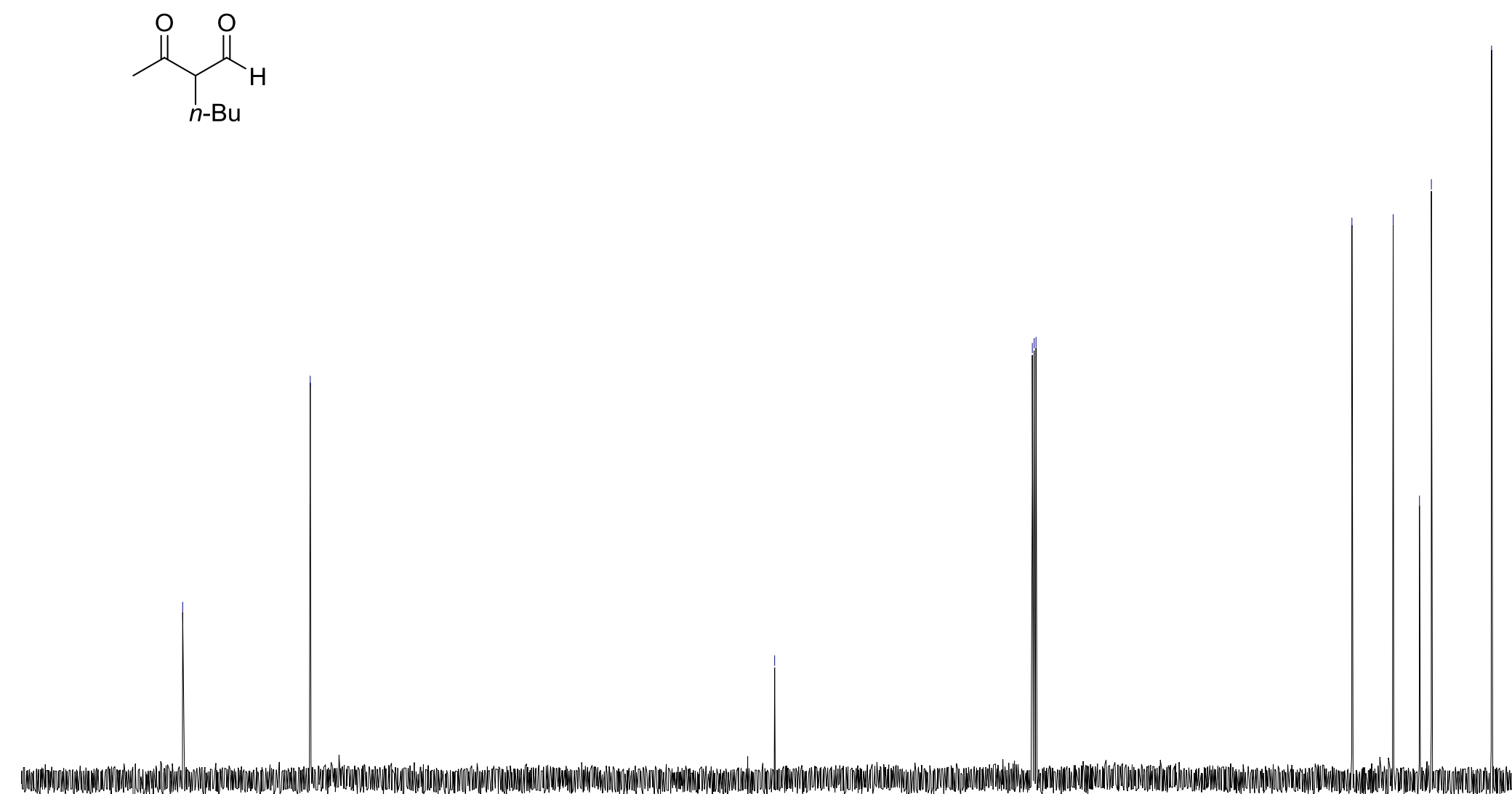

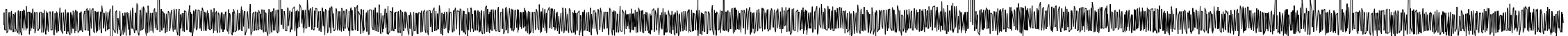

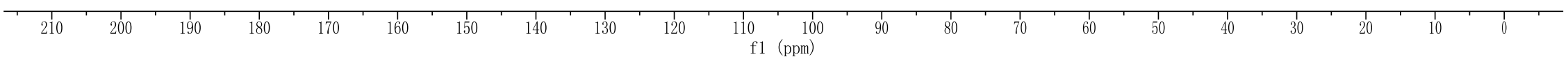




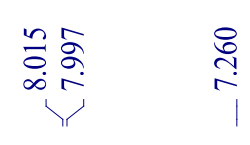

${ }^{1} \mathrm{H} \mathrm{NMR}$ of $8 \mathrm{c} \mathrm{in} \mathrm{CDCl}_{3}$

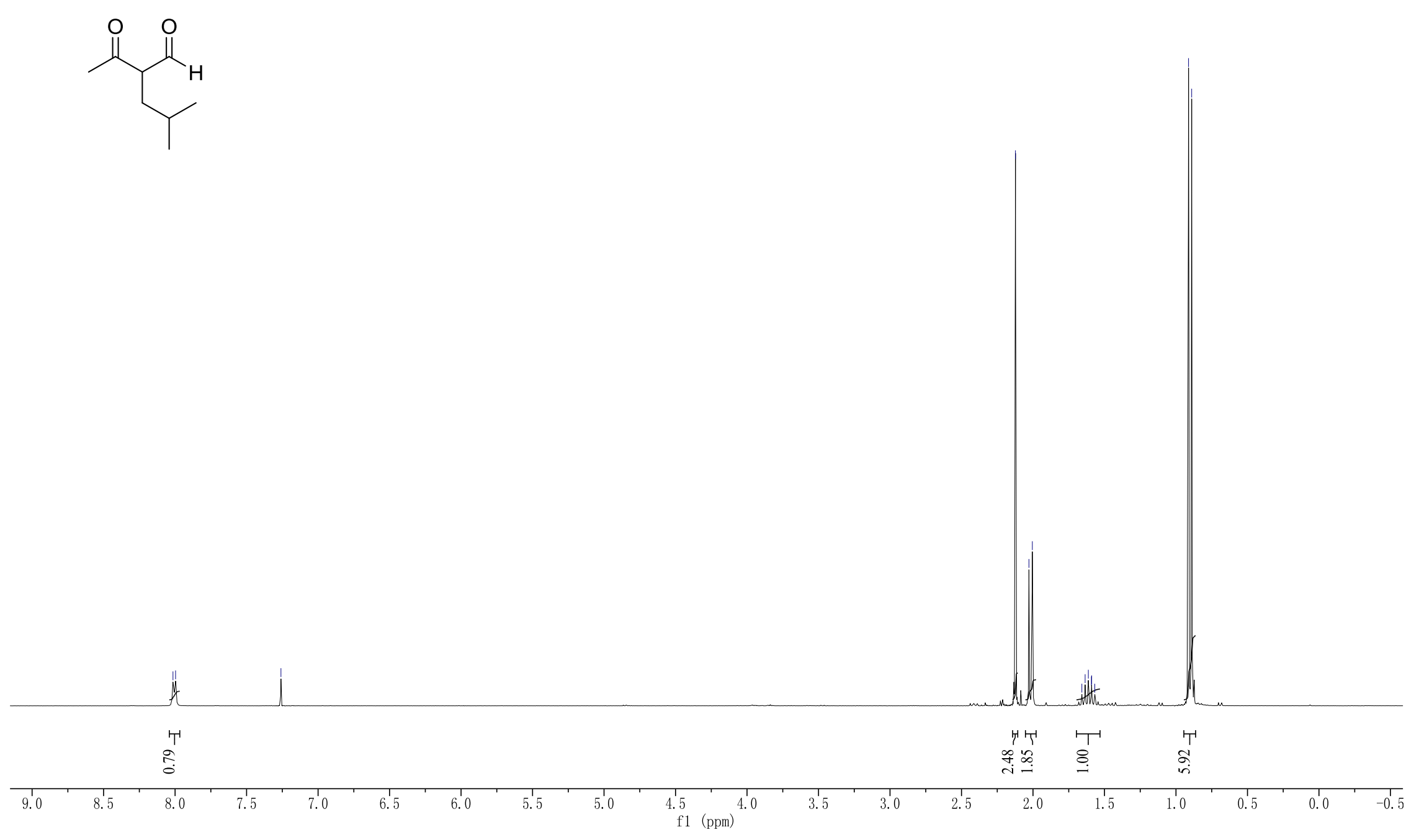




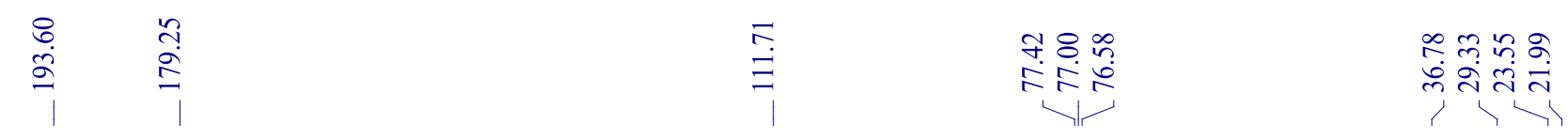

${ }^{13} \mathrm{C}$ NMR of $8 \mathrm{c}$ in $\mathrm{CDCl}_{3}$

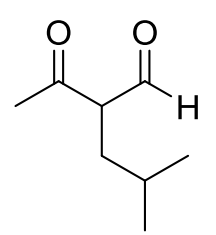

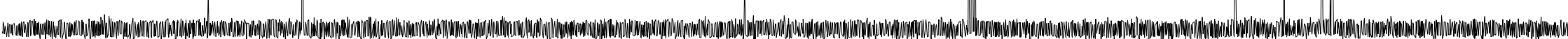

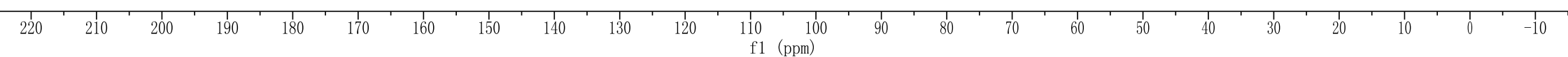




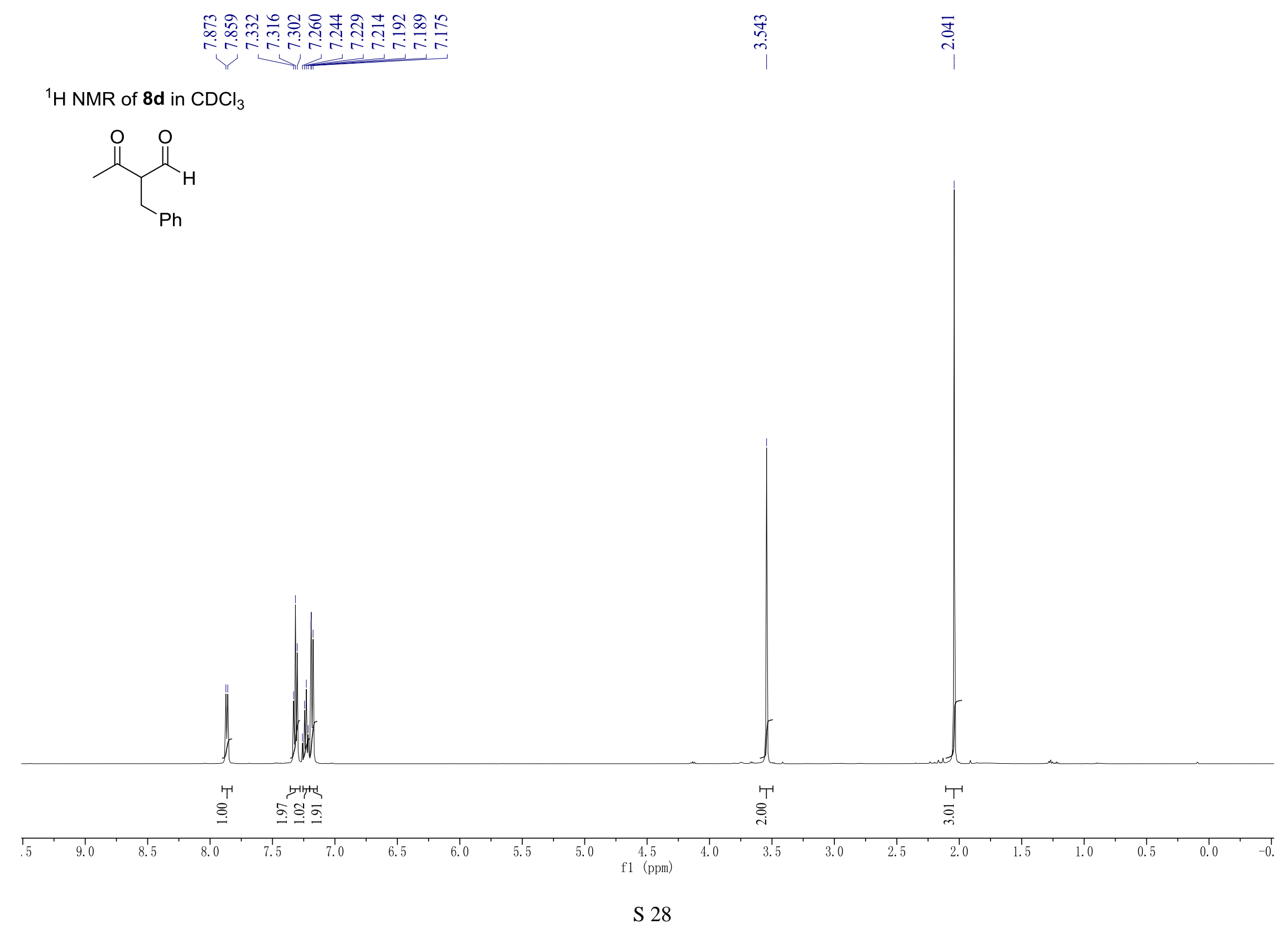




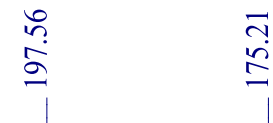

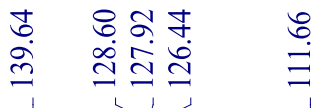
군동
룔 :

${ }^{13} \mathrm{C} \mathrm{NMR}$ of $8 \mathrm{~d}$ in $\mathrm{CDCl}_{3}$<smiles>CC(=O)C(C=O)Cc1ccccc1</smiles>

$\mathrm{Ph}$

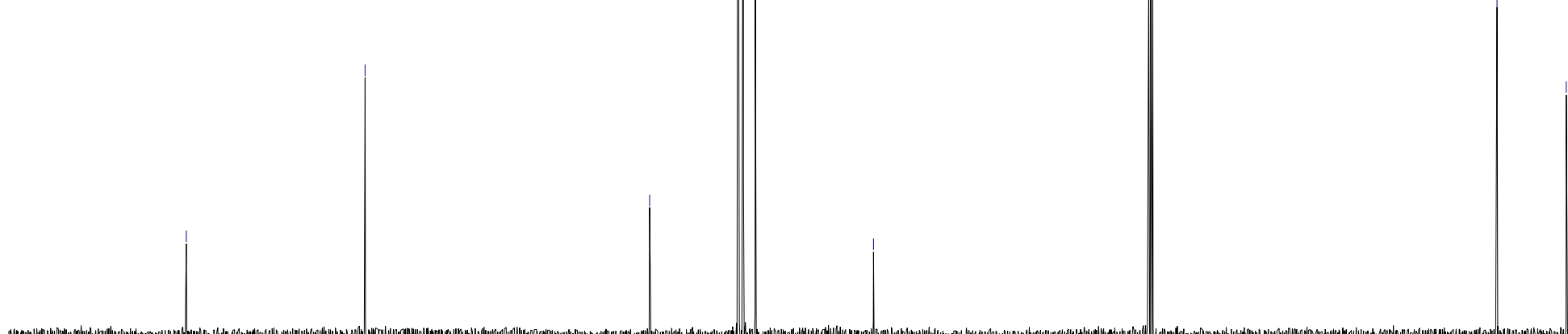

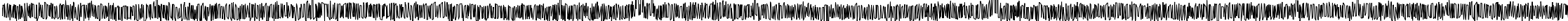

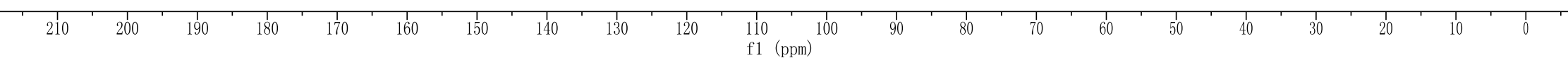




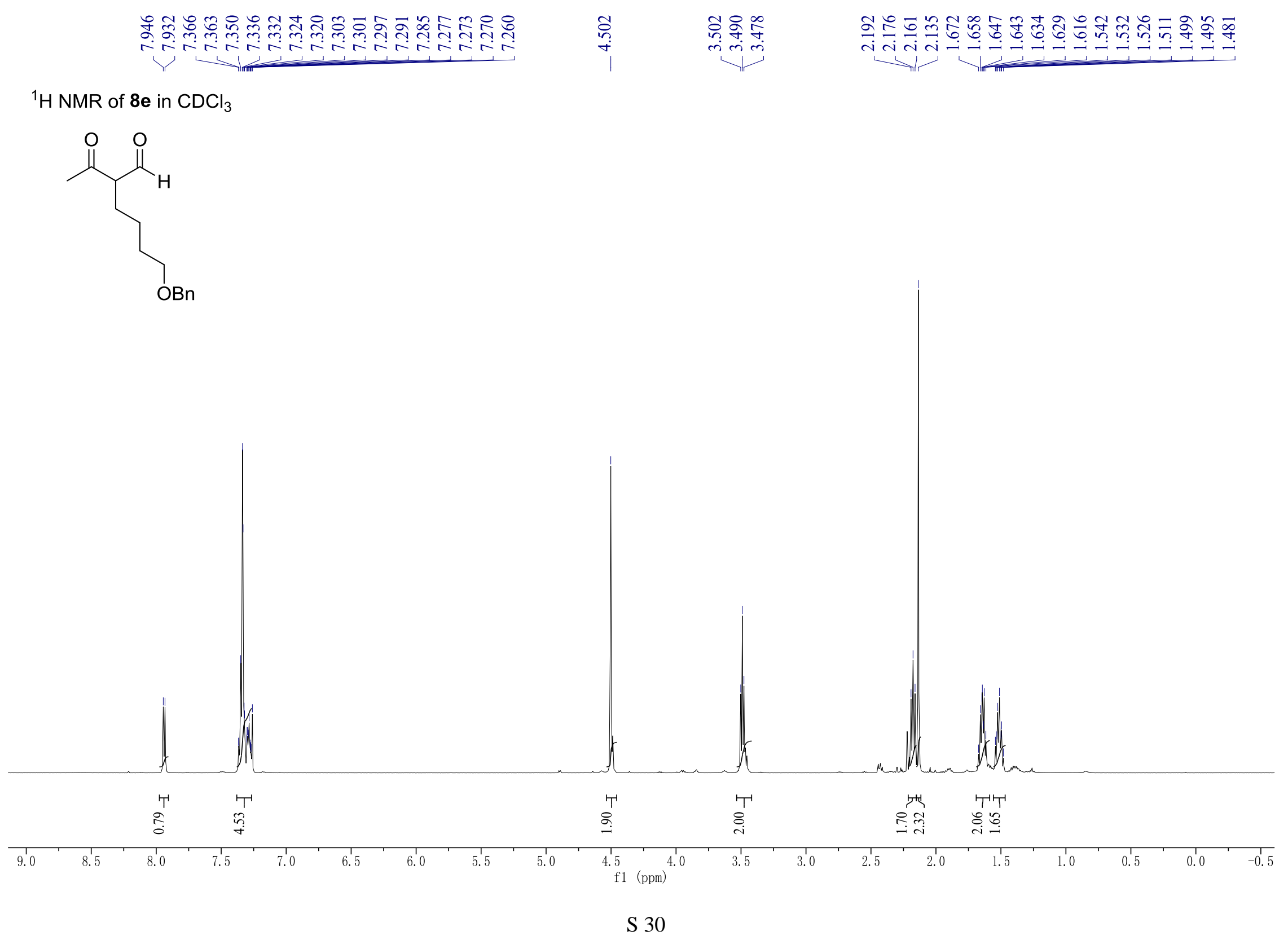



$\stackrel{+}{\stackrel{2}{+}}$

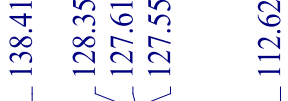

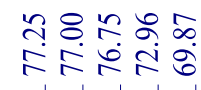

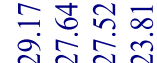

${ }^{13} \mathrm{C} \mathrm{NMR}$ of $8 \mathbf{e}$ in $\mathrm{CDCl}_{3}$

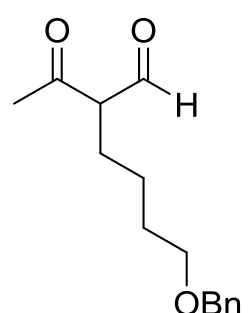

$\mathrm{Bn}$

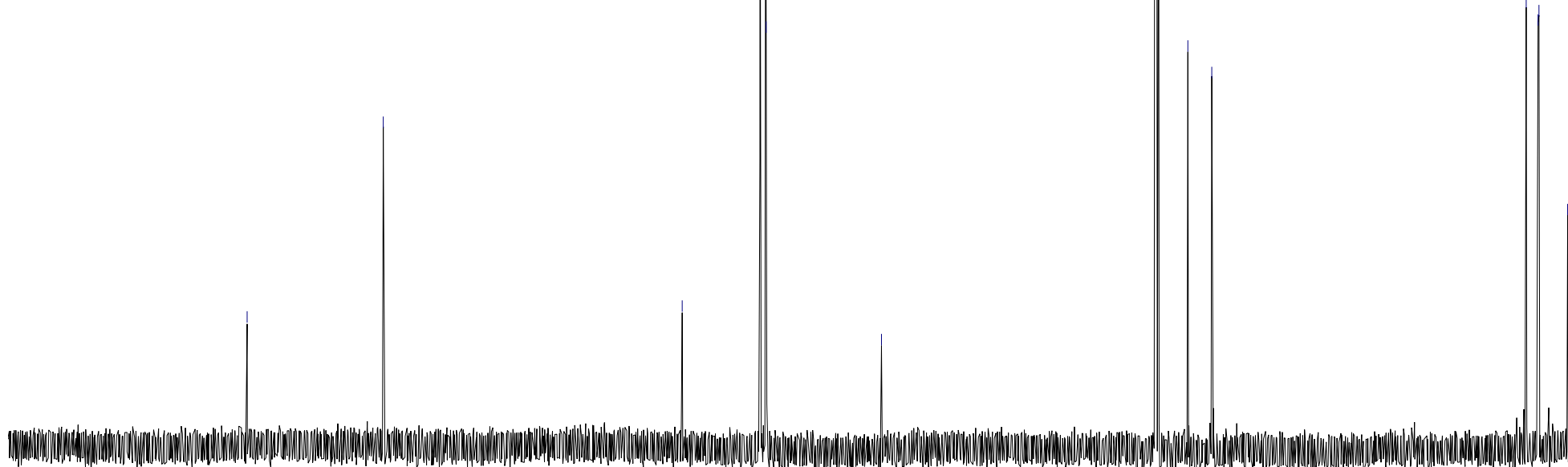

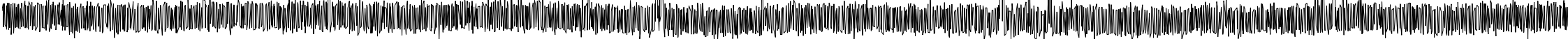

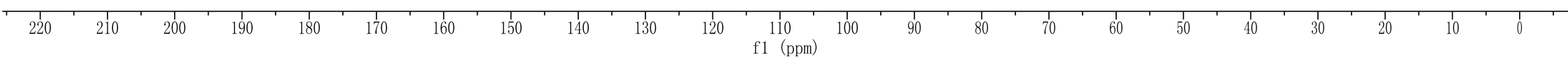




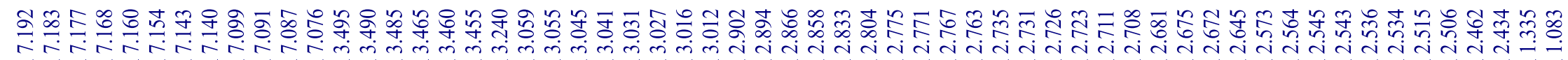
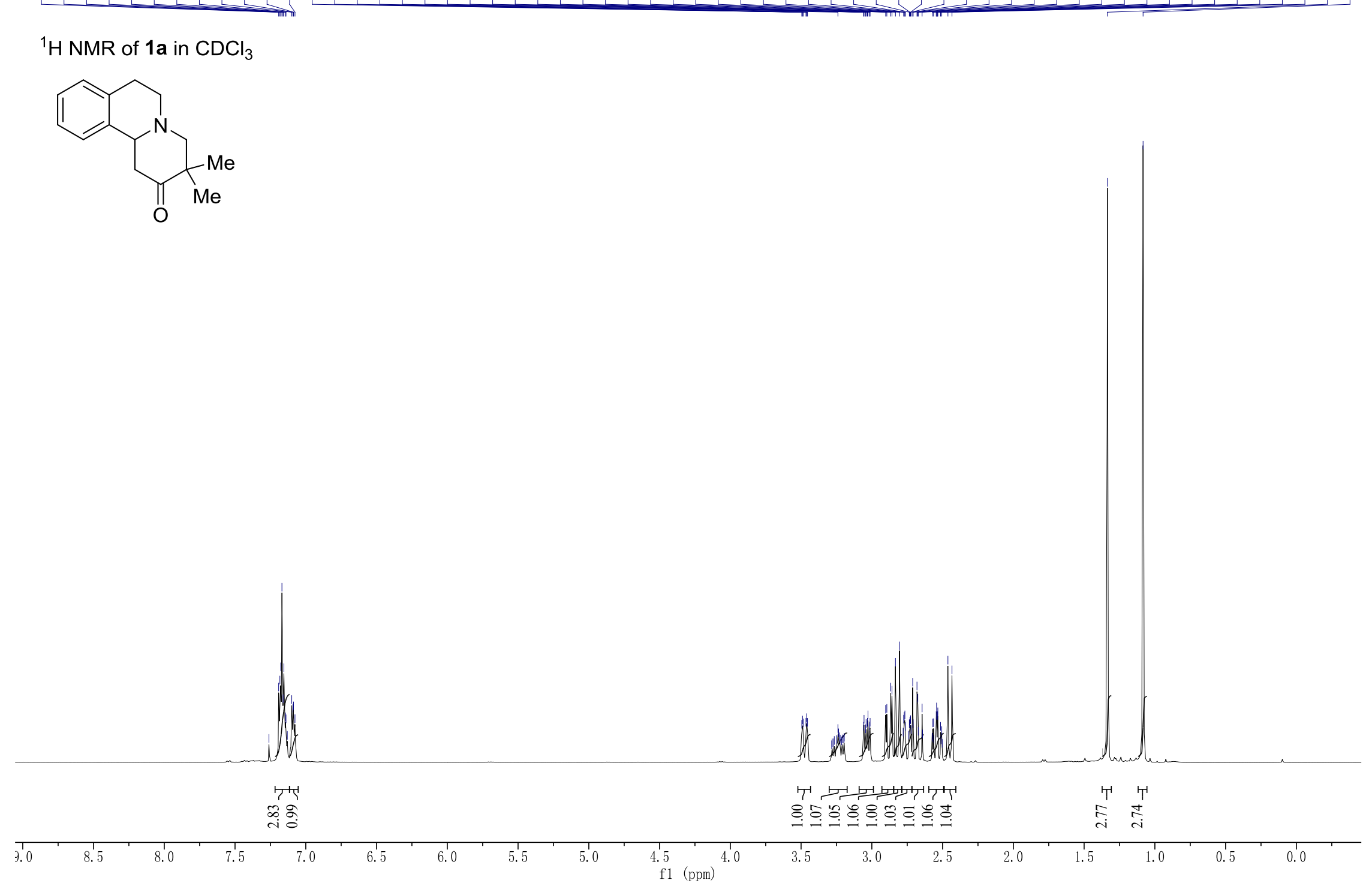


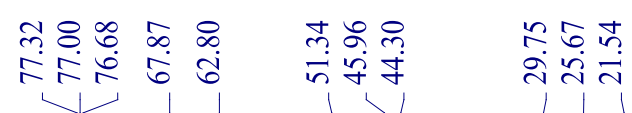

${ }^{13} \mathrm{C}$ NMR of $1 \mathrm{a}$ in $\mathrm{CDCl}_{3}$
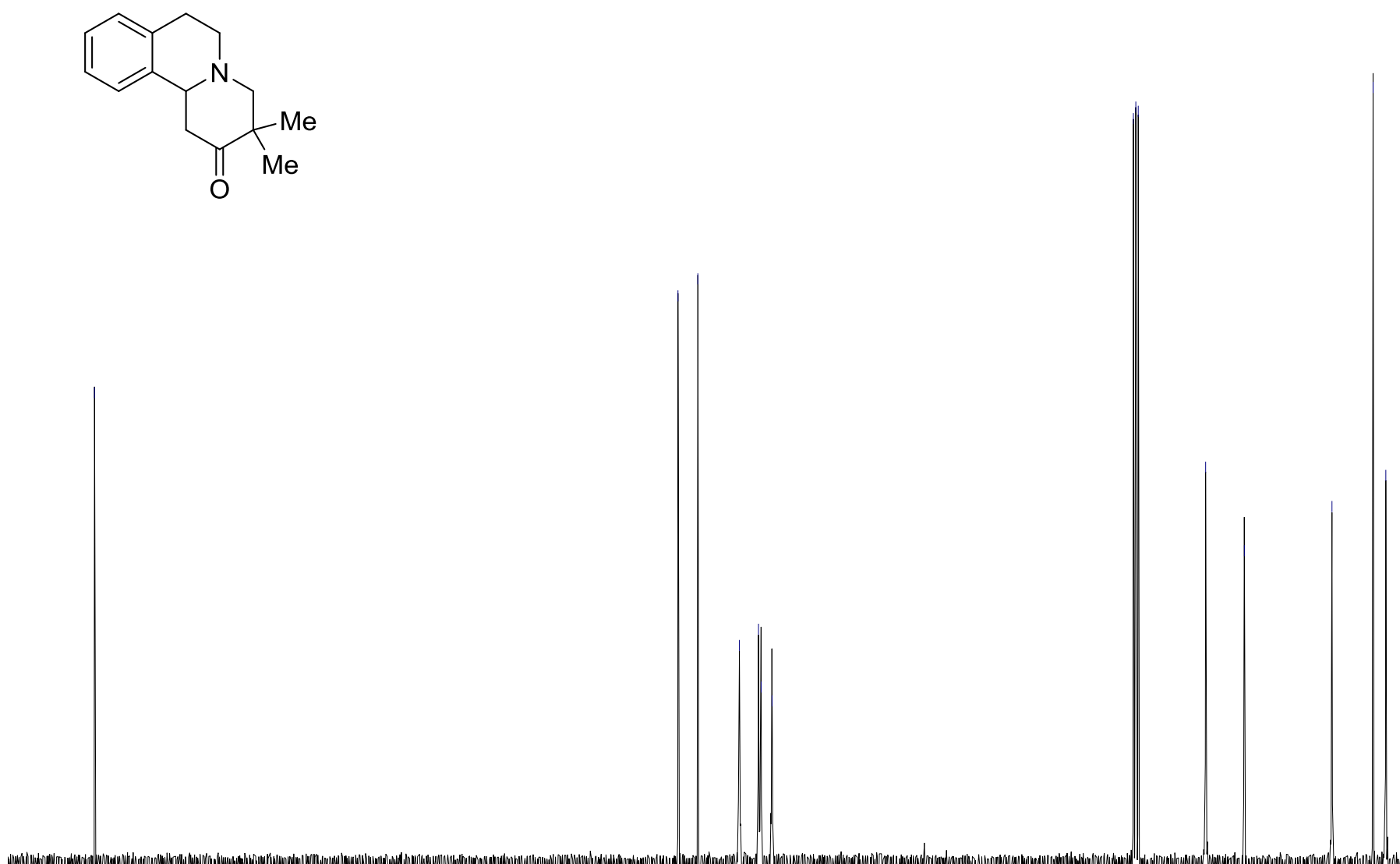

y.w. 


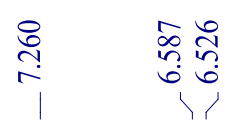

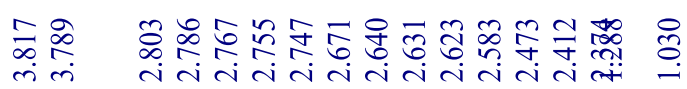

${ }^{1} \mathrm{H}$ NMR of $\mathbf{1 b}$ in $\mathrm{CDCl}_{3}$
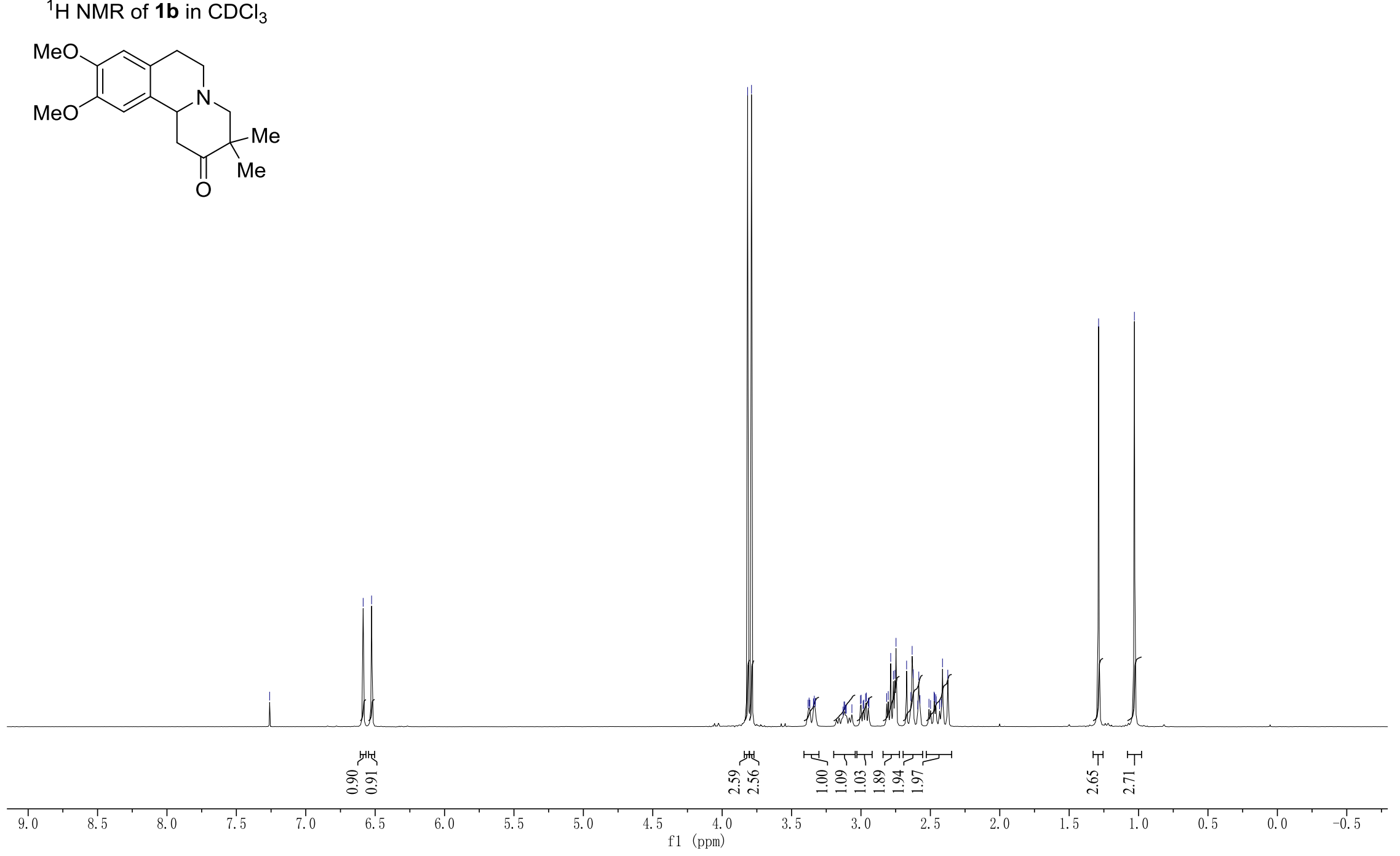


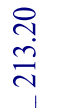
导先

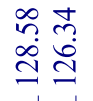

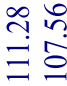

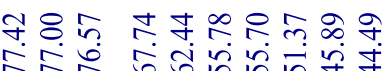
$\vec{\pi} \infty$

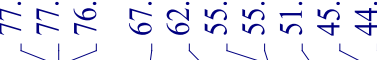
กิ่

${ }^{13} \mathrm{C}$ NMR of $\mathbf{1 b}$ in $\mathrm{CDCl}_{3}$

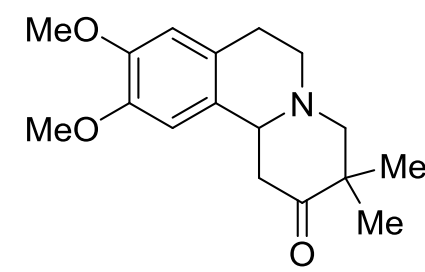

\section{Me}

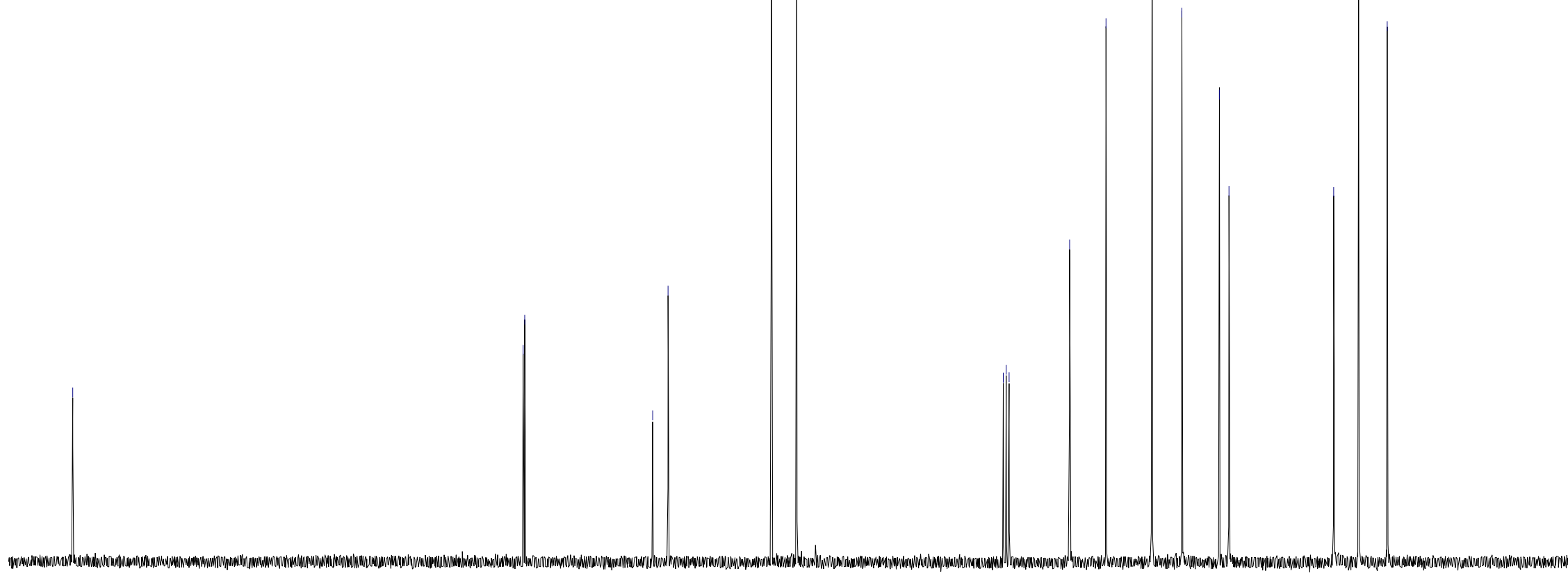

$\frac{1}{220}$
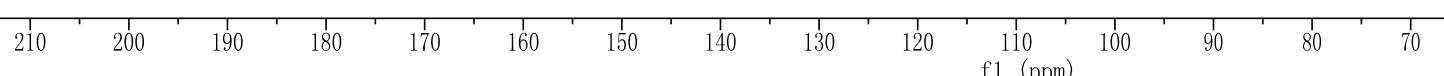

60
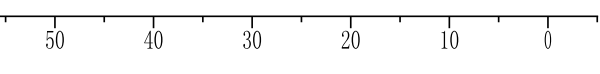


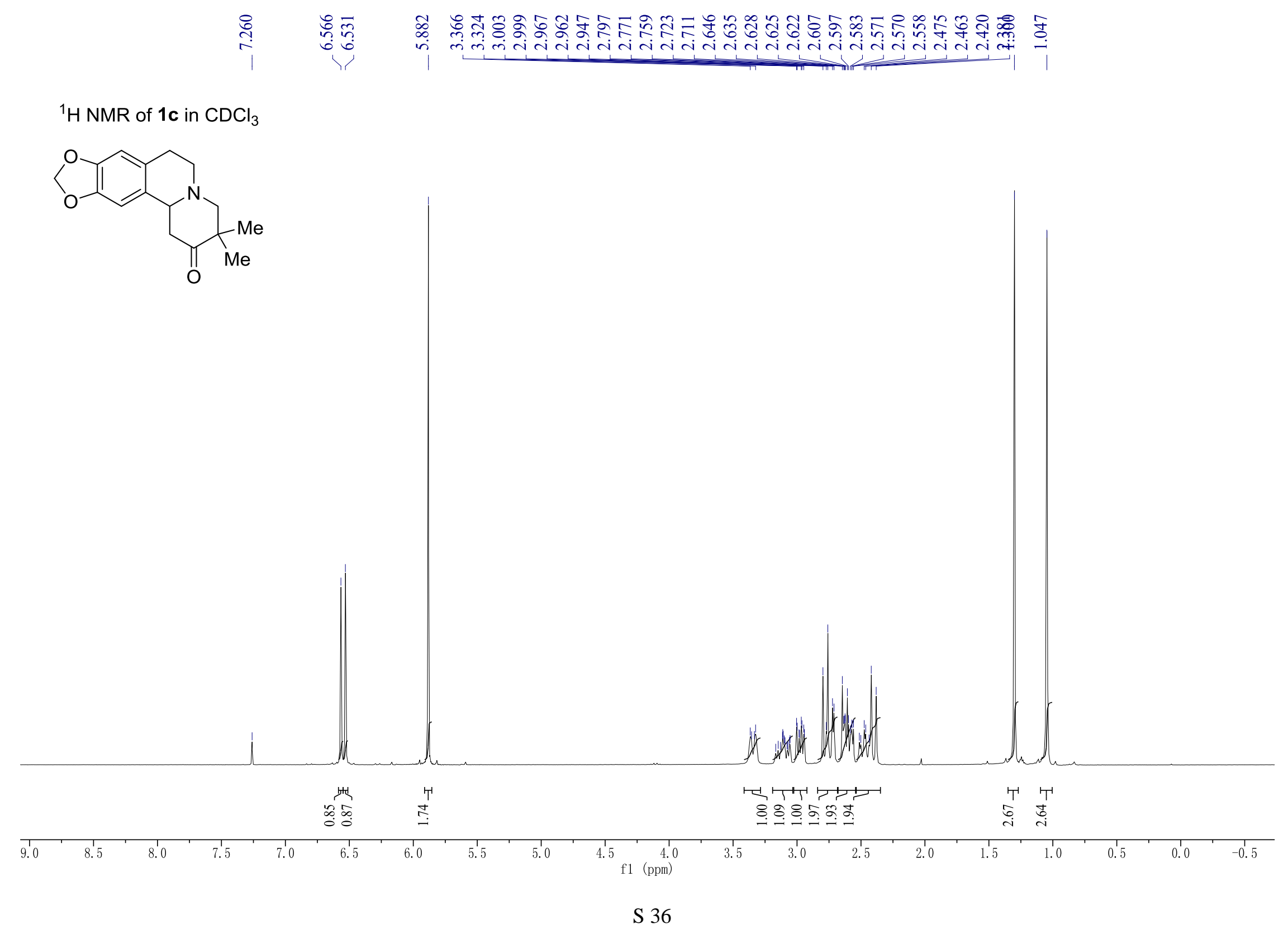


ปे

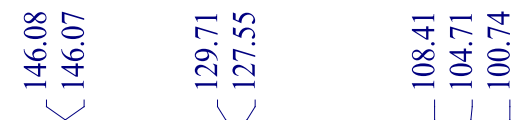

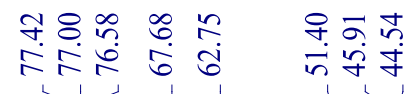

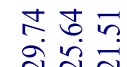

${ }^{13} \mathrm{C} \mathrm{NMR}$ of $1 \mathrm{c}$ in $\mathrm{CDCl}_{3}$
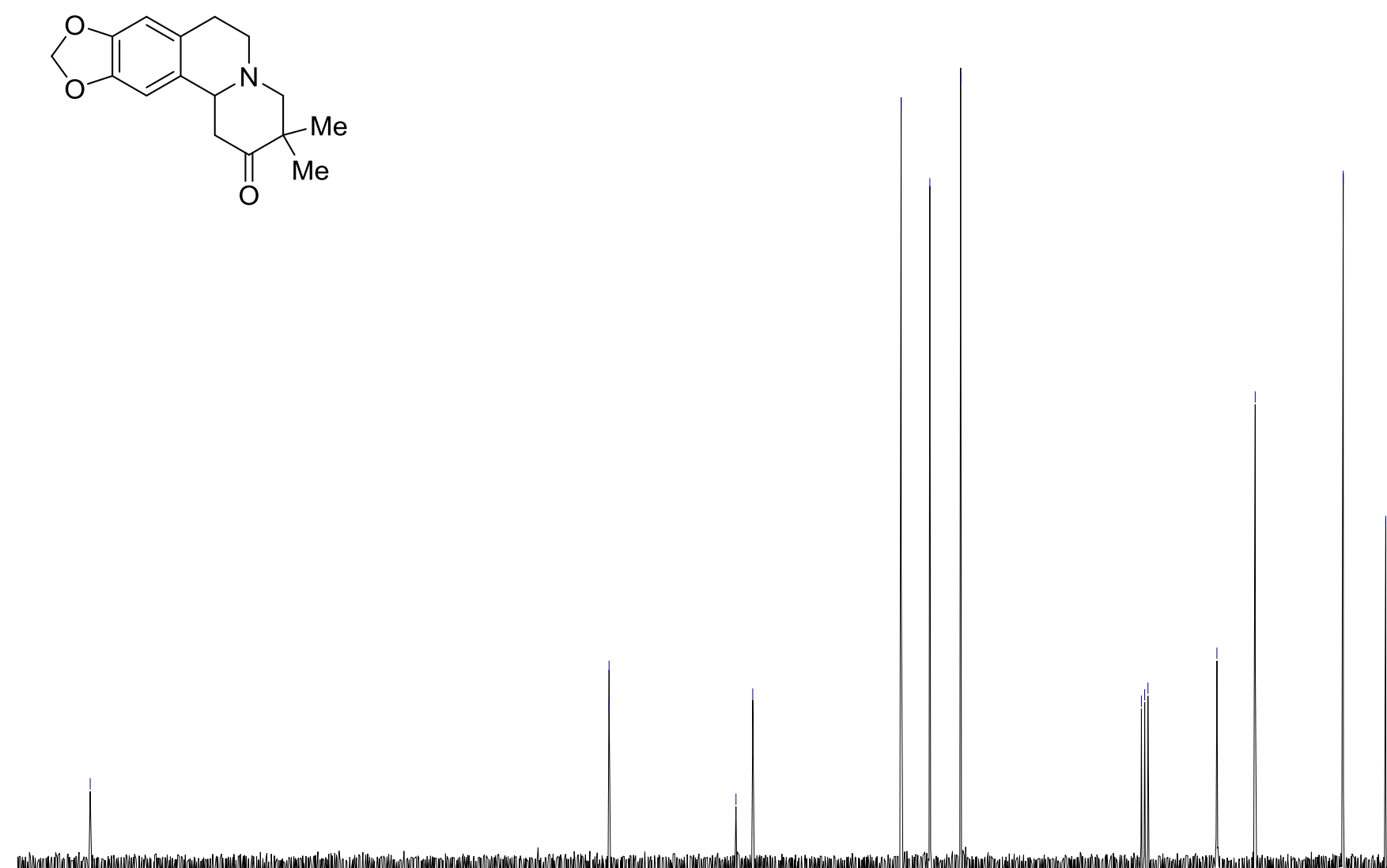

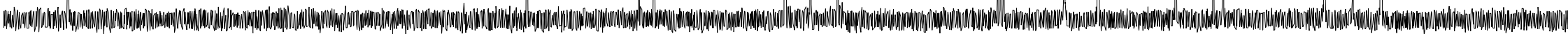

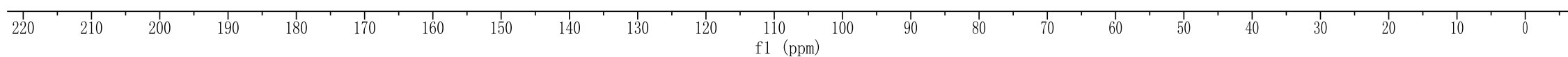


${ }^{1} \mathrm{H} \mathrm{NMR}$ of $\mathbf{1 d}$ in $\mathrm{CDCl}_{3}$
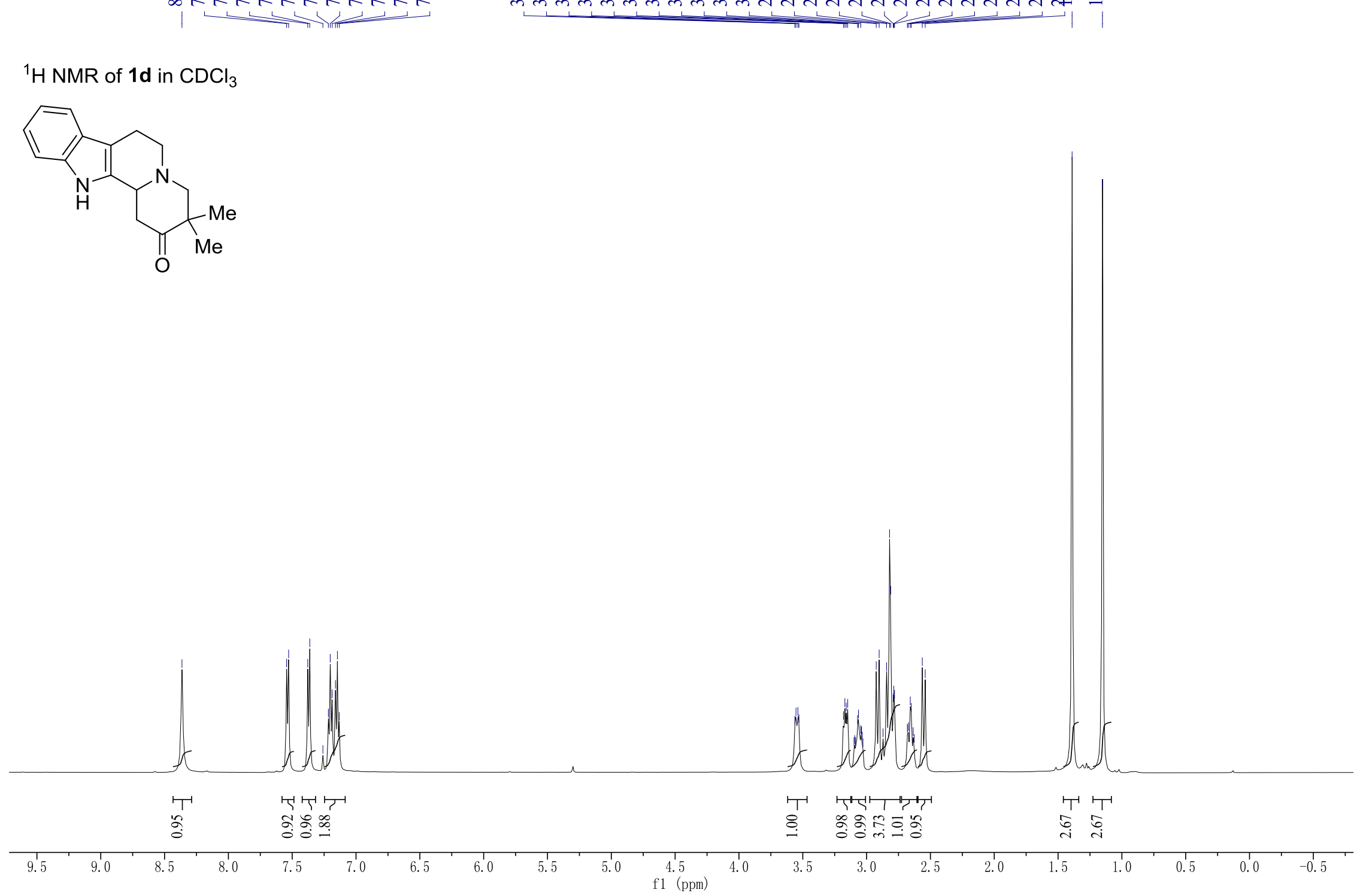


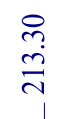

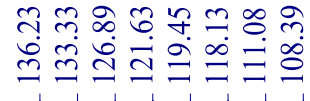

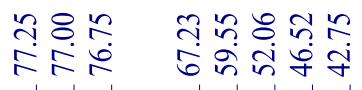

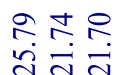

${ }^{13} \mathrm{C}$ NMR of $1 \mathbf{d}$ in $\mathrm{CDCl}_{3}$

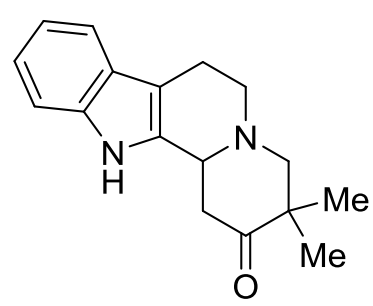

$\mid$

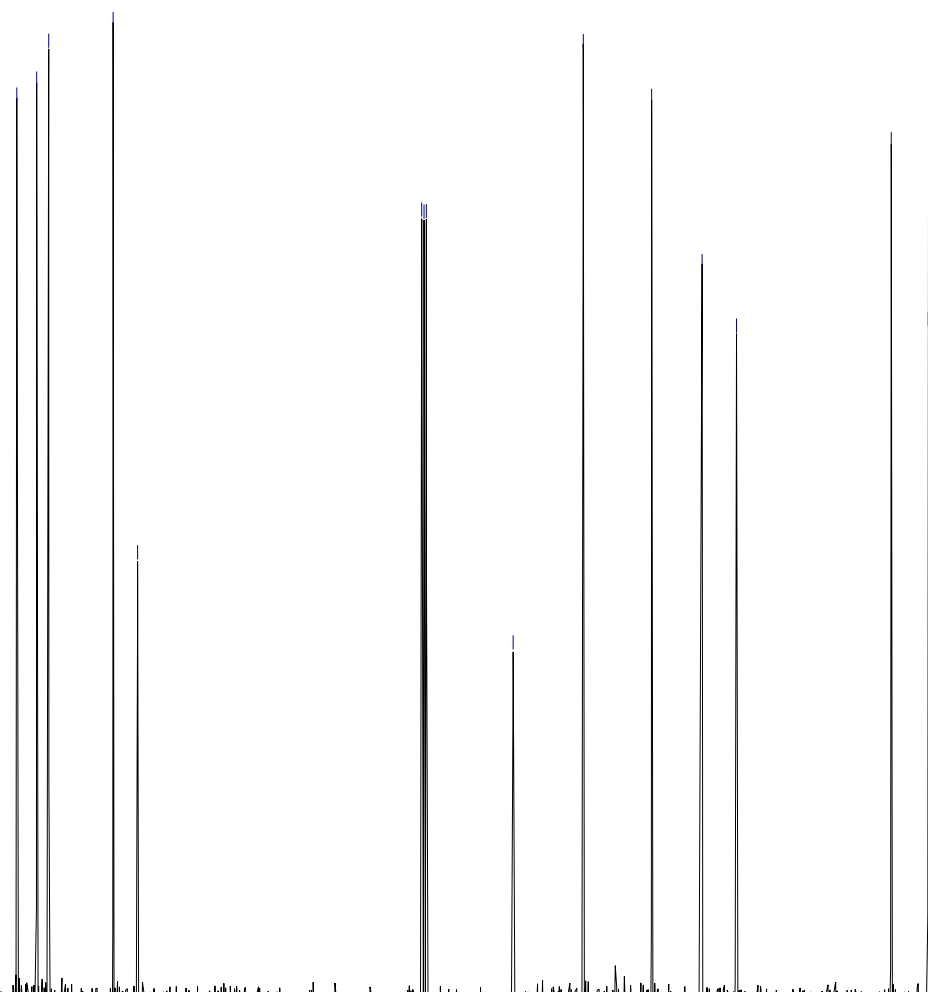

Ind

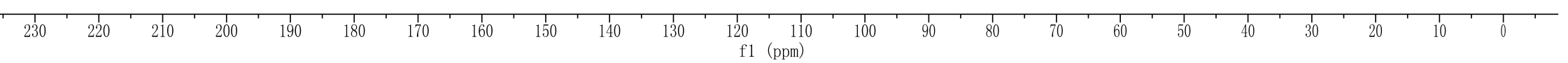



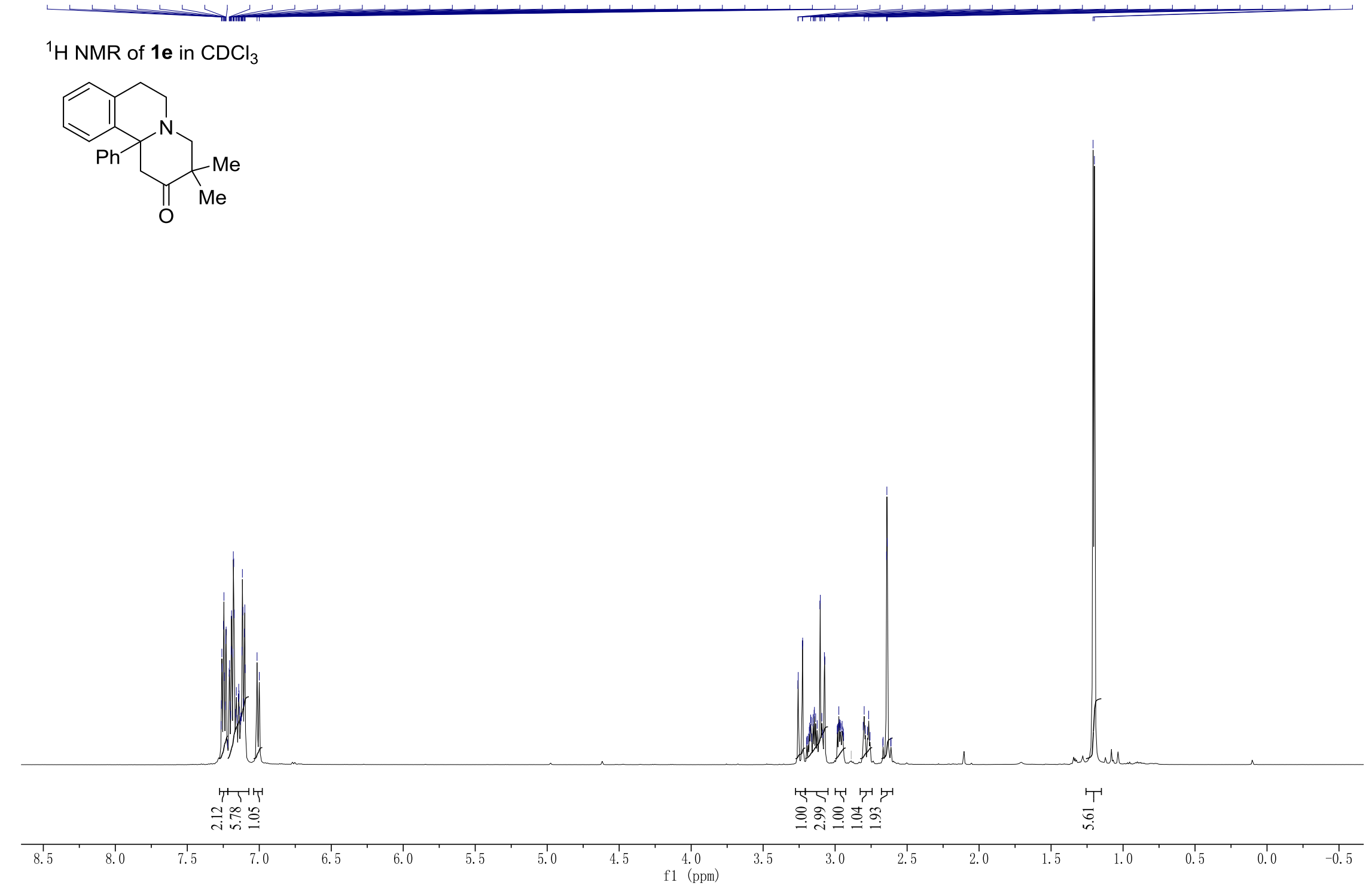


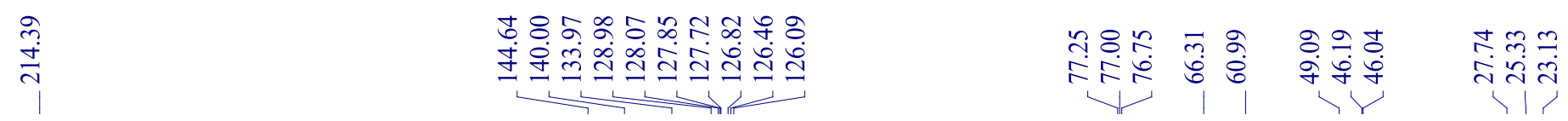

${ }^{13} \mathrm{C} \mathrm{NMR}$ of $1 \mathrm{e}$ in $\mathrm{CDCl}_{3}$
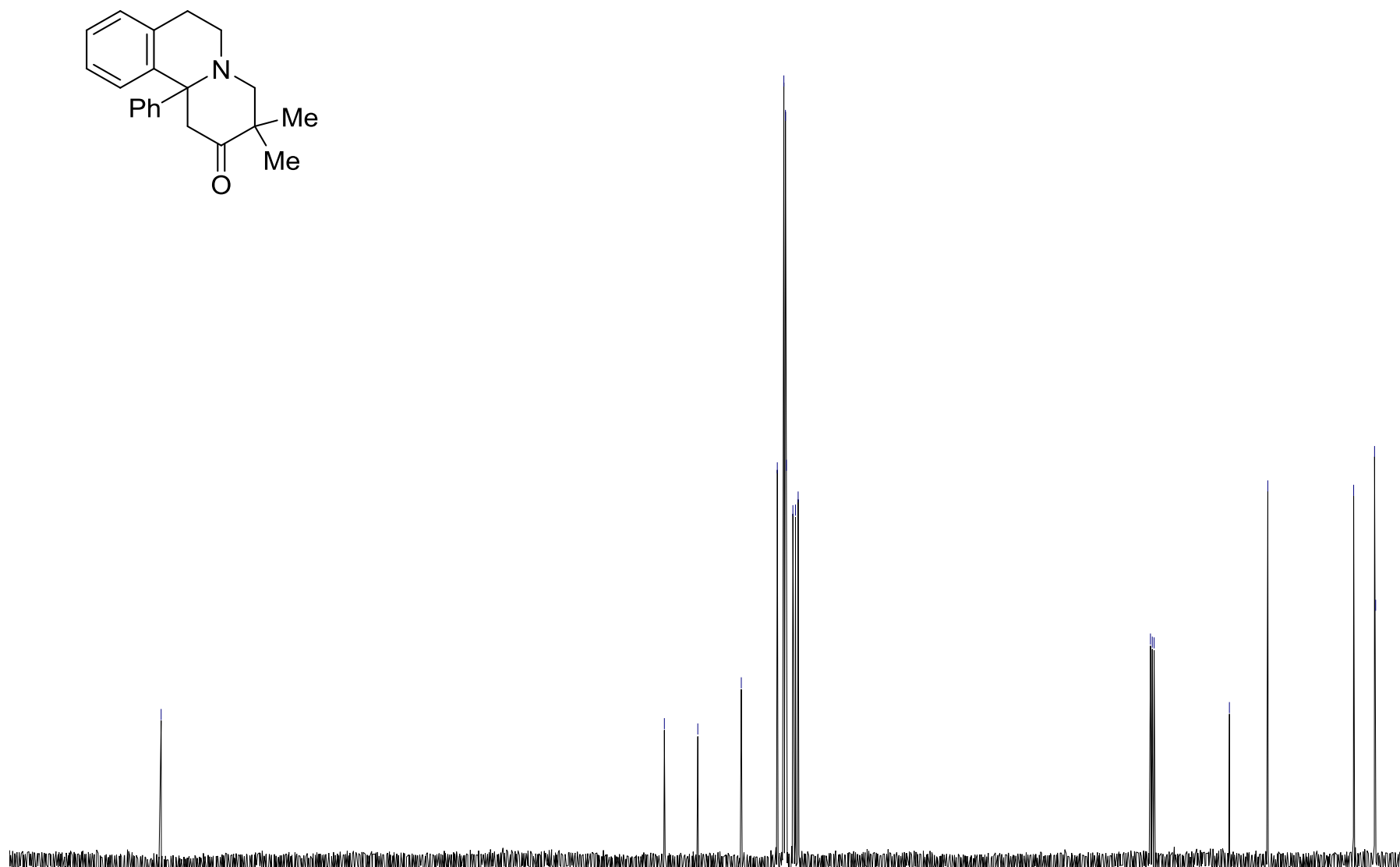

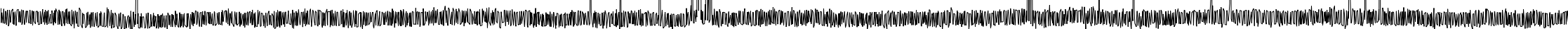

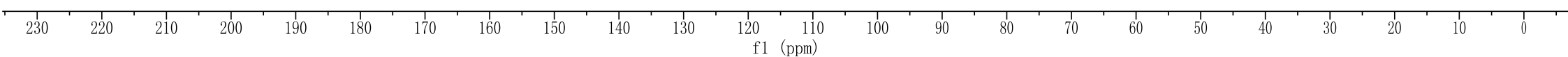




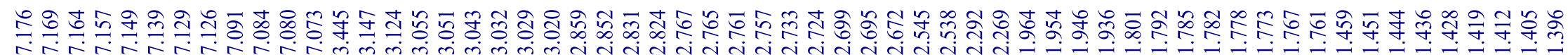
1 ${ }^{1} \mathrm{H} \mathrm{NMR}$ of $1 \mathrm{f}$ in $\mathrm{CDCl}_{3}$

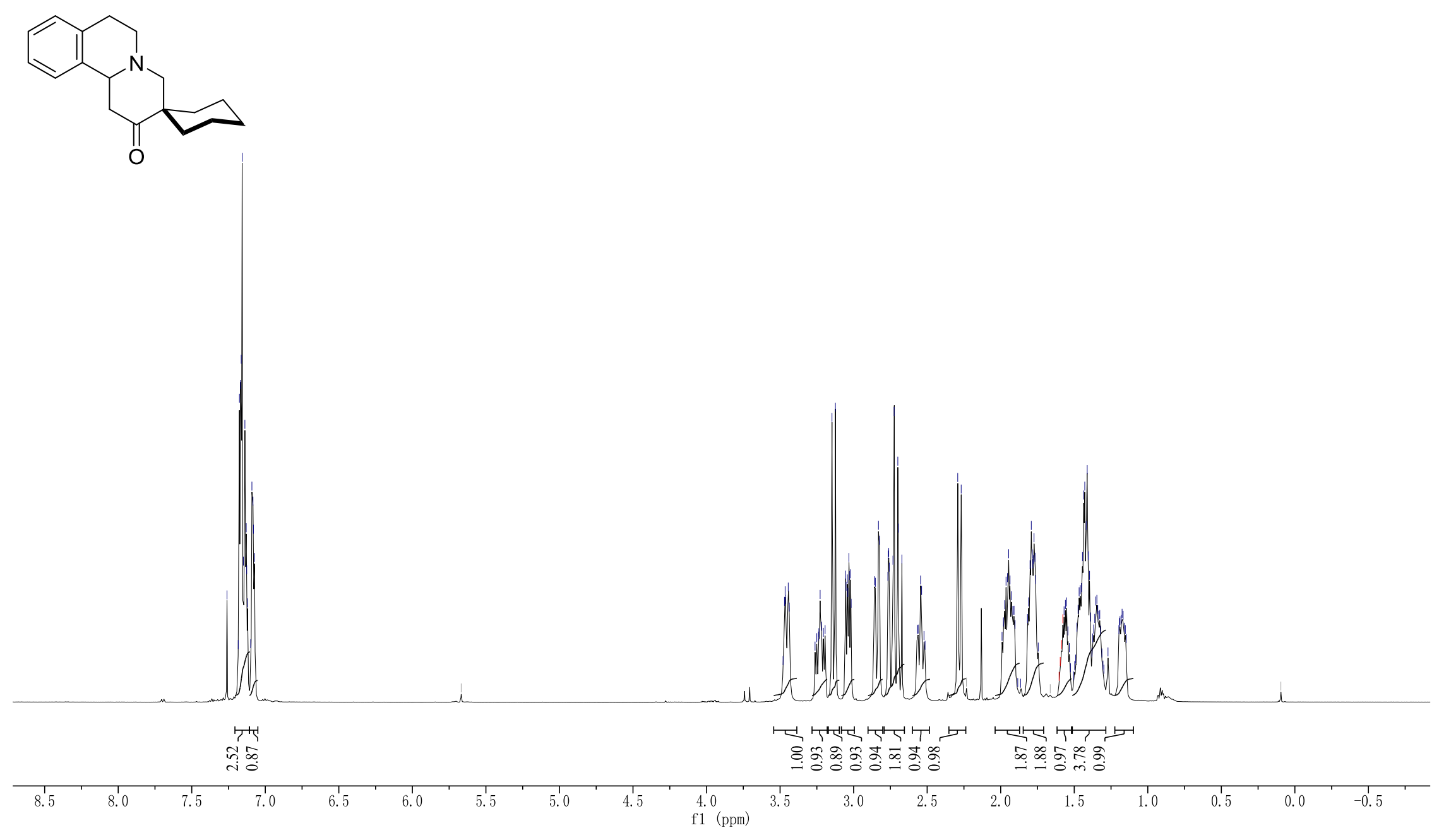




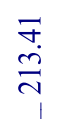

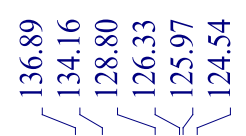

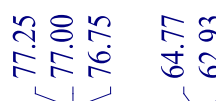

กำ

${ }^{13} \mathrm{C} \mathrm{NMR}$ of $1 \mathrm{f}$ in $\mathrm{CDCl}_{3}$

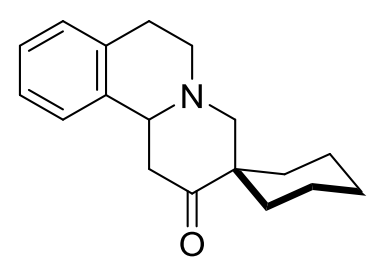

O

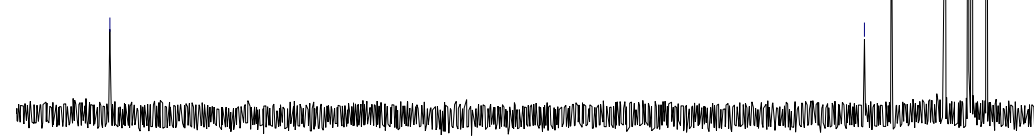
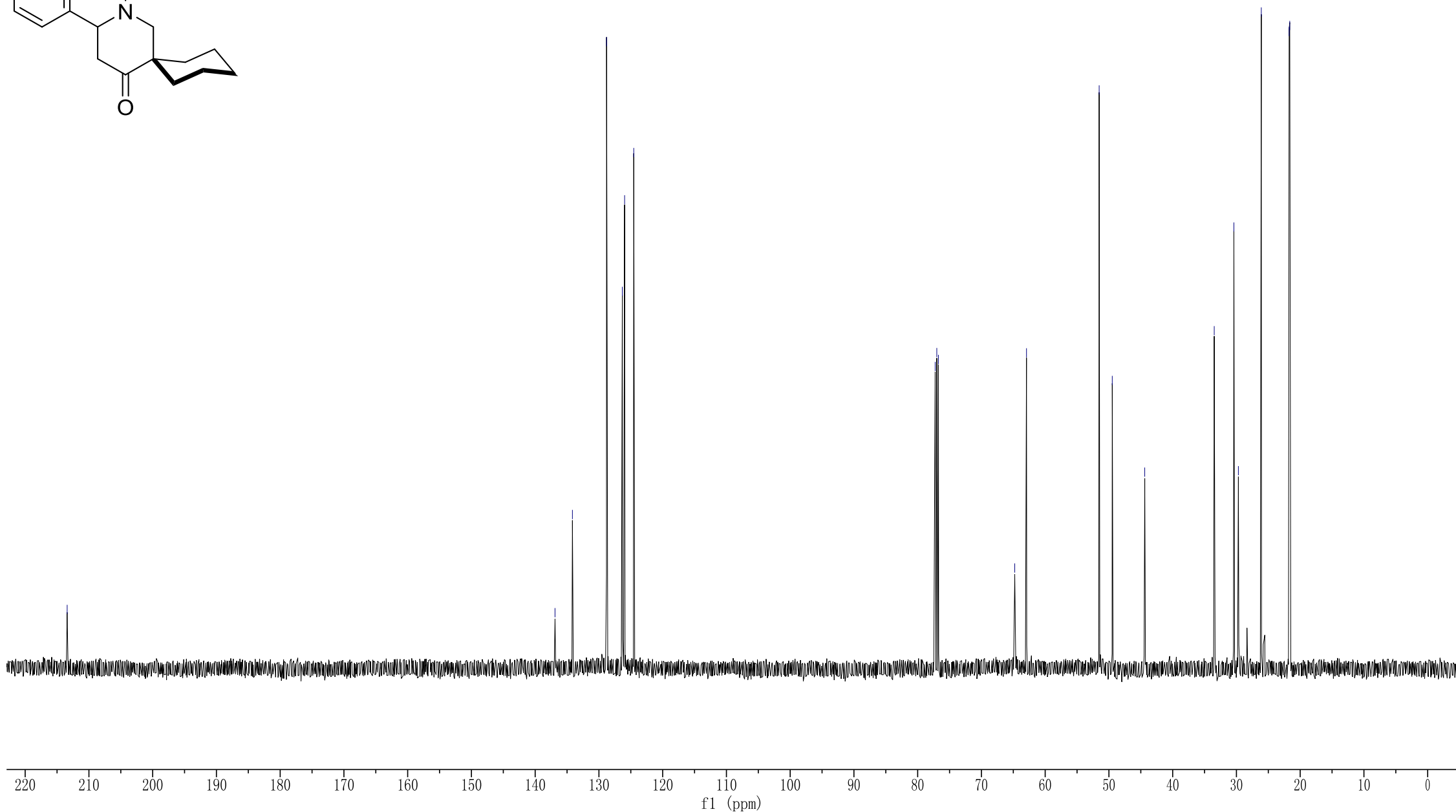


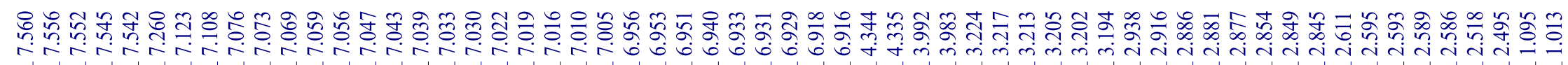
${ }^{1} \mathrm{H}$ NMR of cis-19 in $\mathrm{CDCl}_{3}$
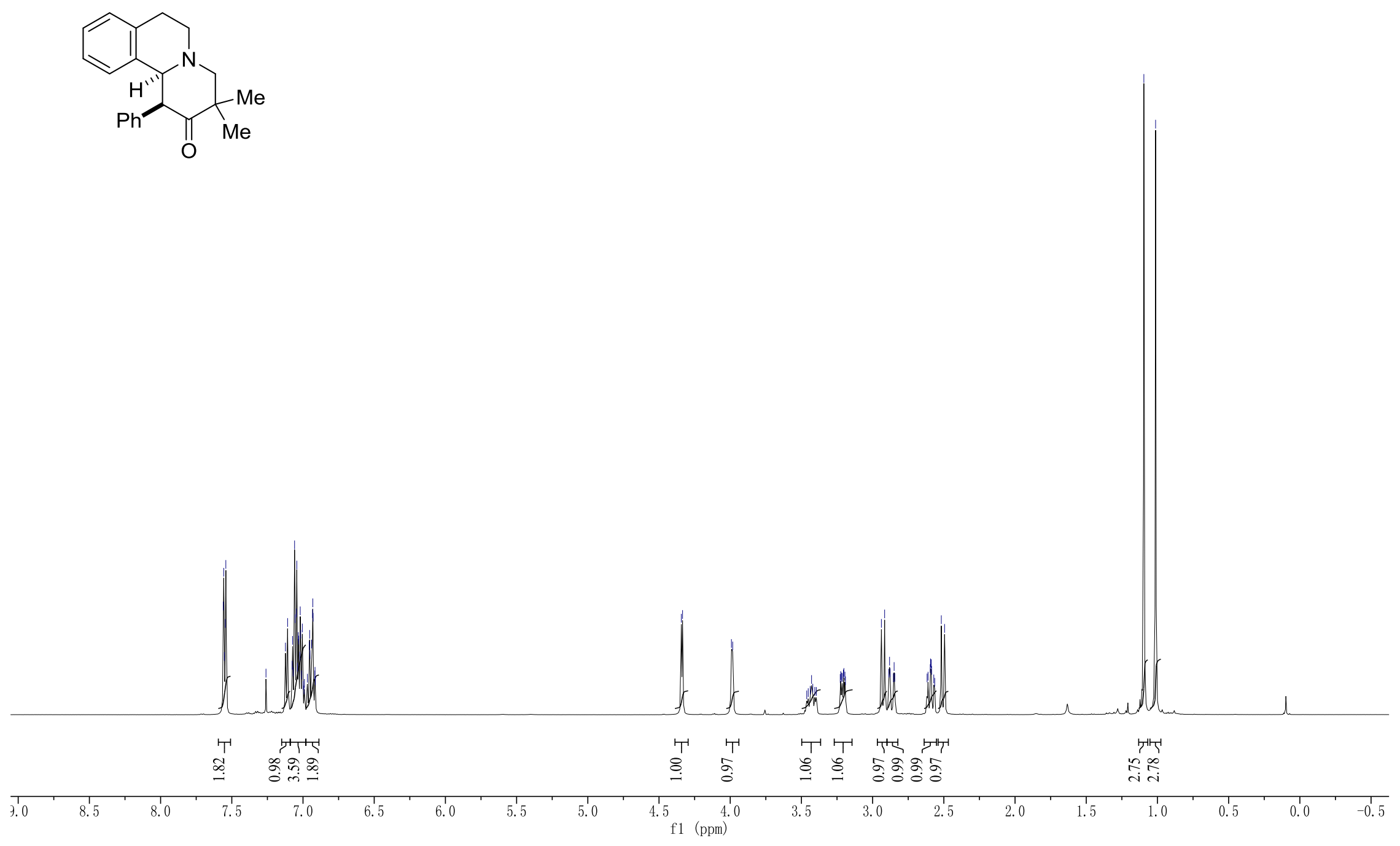


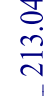
흐유은으영

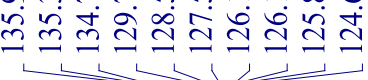

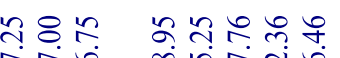

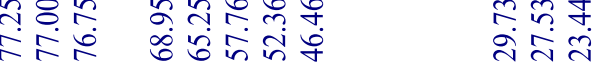

${ }^{13} \mathrm{C} \mathrm{NMR}$ of cis-1 $\mathbf{g}$ in $\mathrm{CDCl}_{3}$

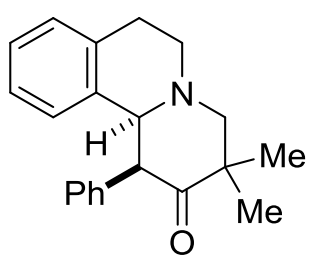

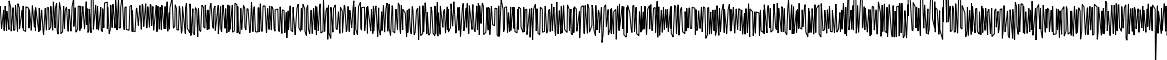

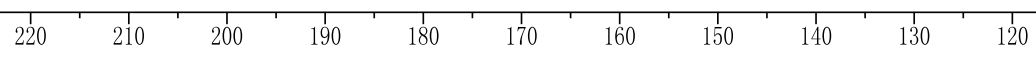

1 (ppm) 


\section{${ }^{1} \mathrm{H}$ NMR of trans-19 in $\mathrm{CDCl}_{3}$}
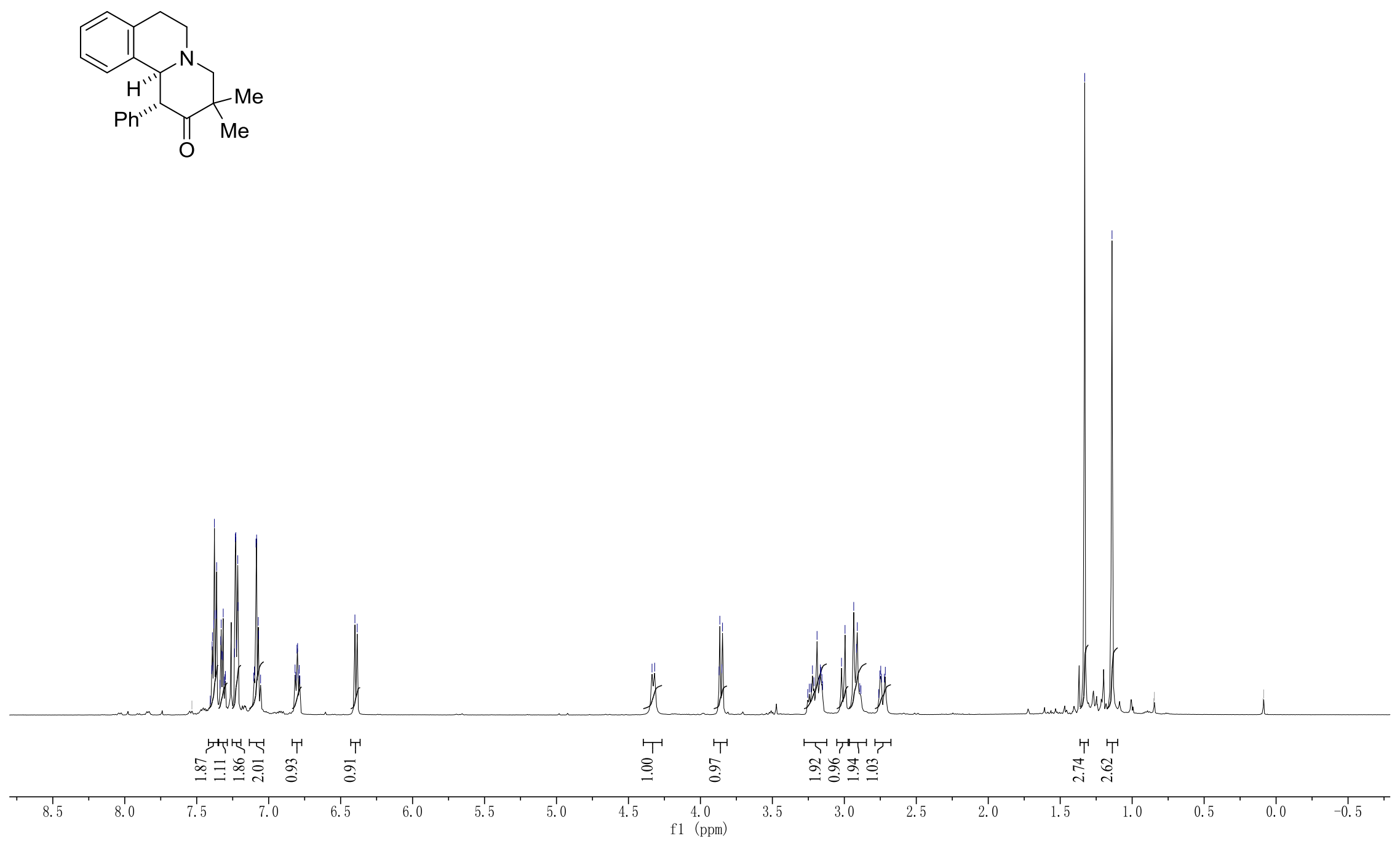


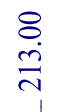

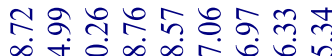

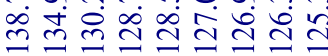

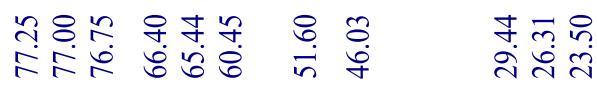

${ }^{13} \mathrm{C}$ NMR of trans-19 in $\mathrm{CDCl}_{3}$

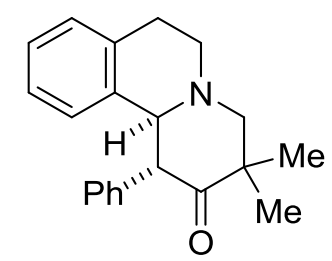

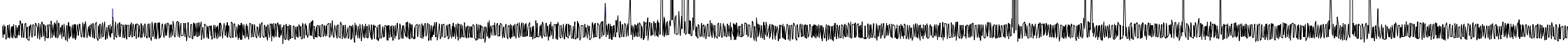

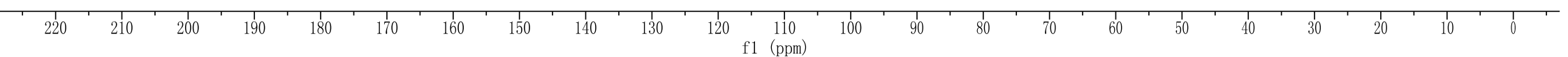




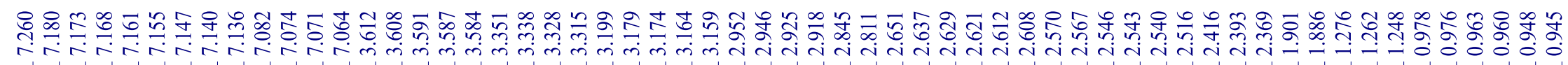
${ }^{1} \mathrm{H}$ NMR of $1 \mathrm{~h}$ in $\mathrm{CDCl}_{3}$
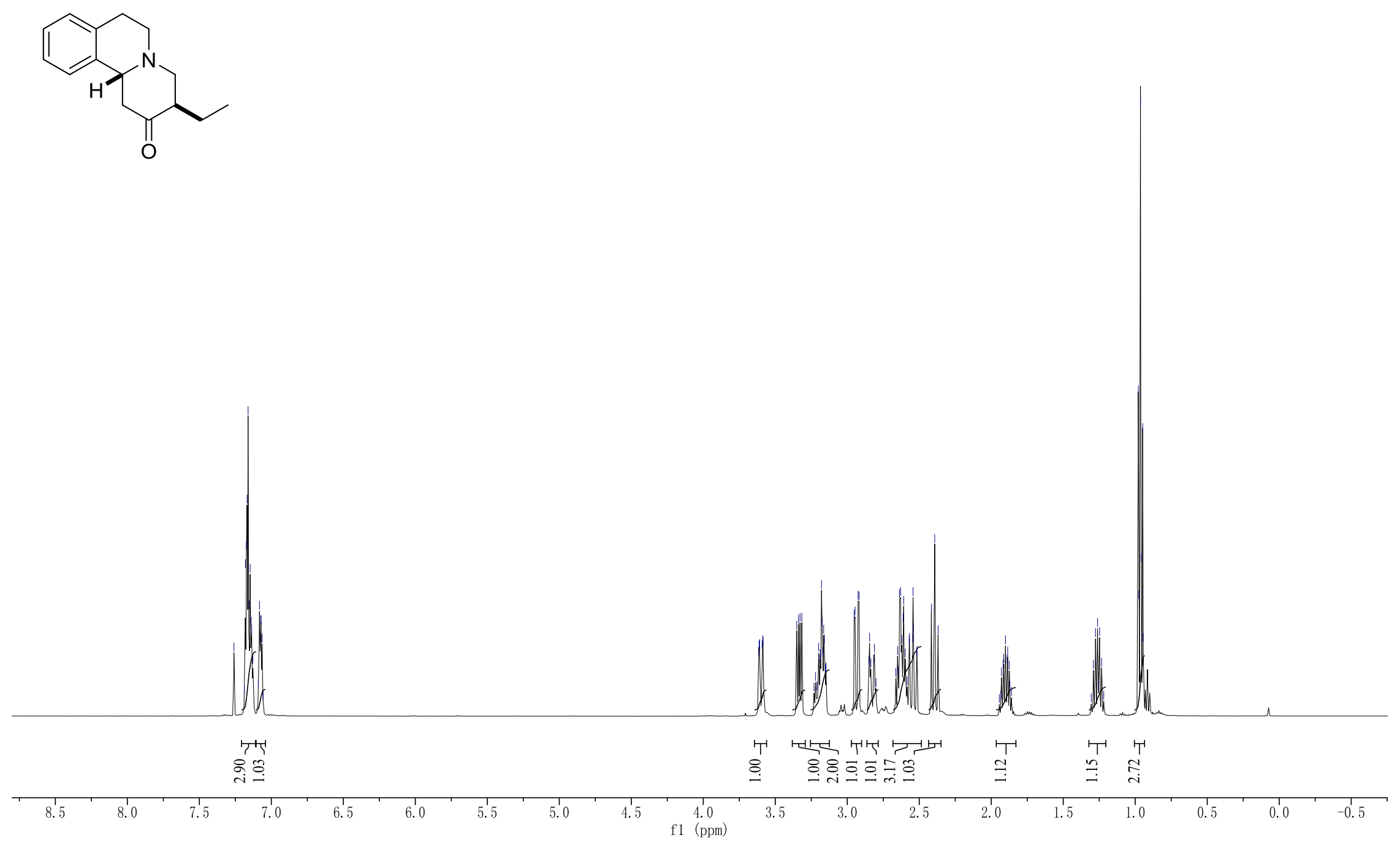
คั.

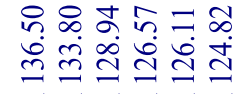

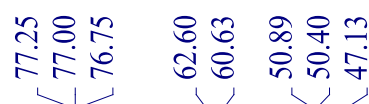

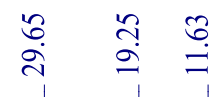

${ }^{13} \mathrm{C} \mathrm{NMR}$ of $1 \mathrm{~h}$ in $\mathrm{CDCl}_{3}$

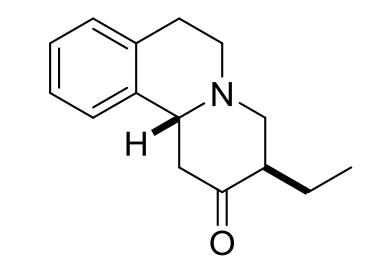

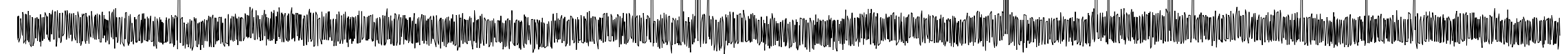

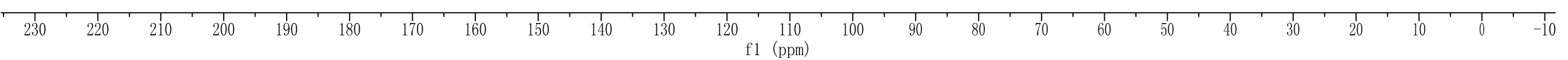




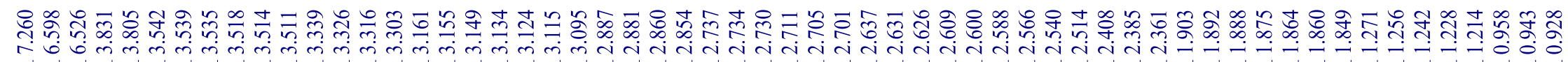

\section{${ }^{1} \mathrm{H} \mathrm{NMR}$ of $1 \mathrm{i}$ in $\mathrm{CDCl}_{3}$}
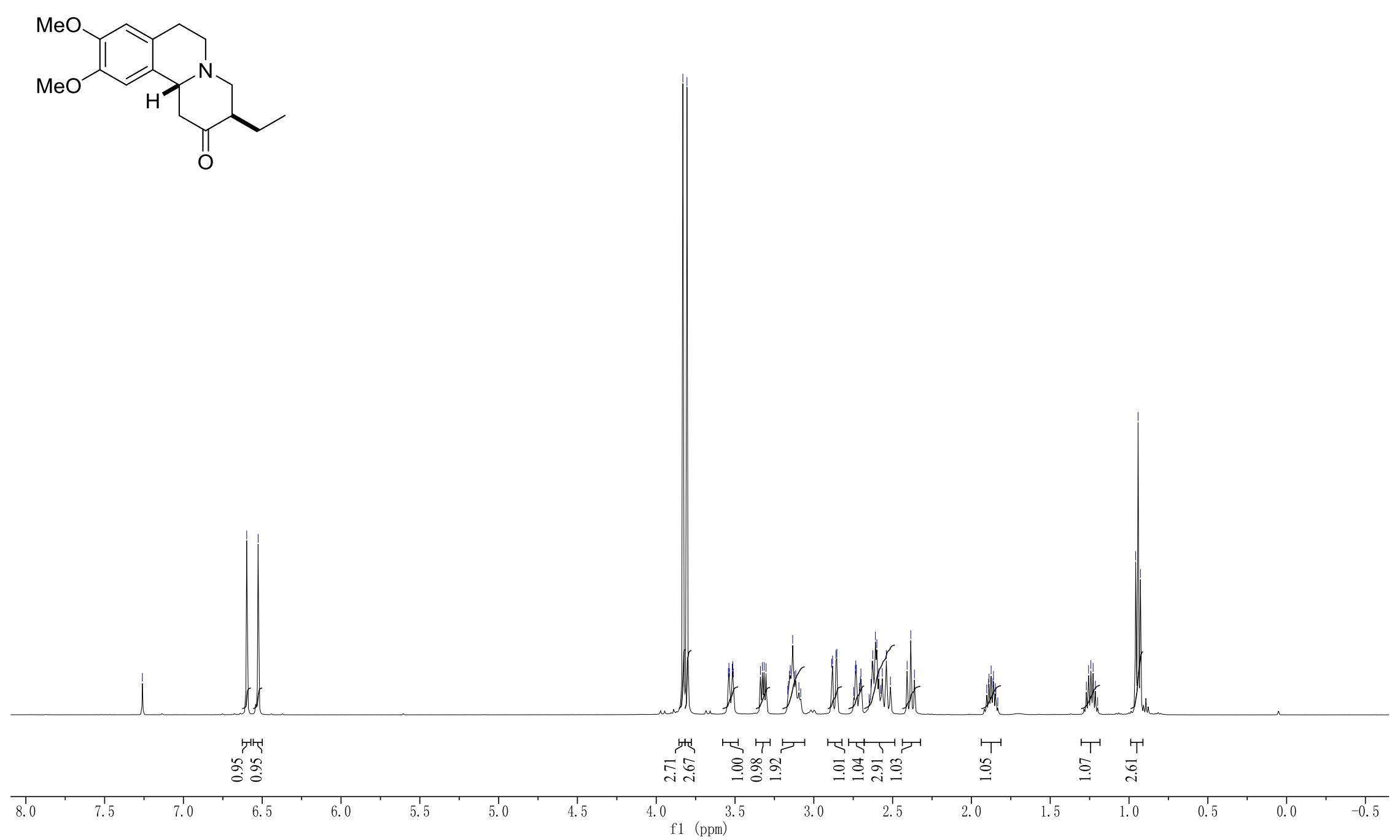

㓠.

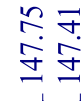

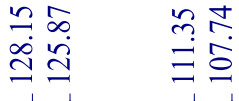

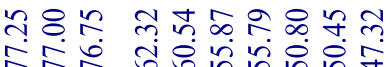

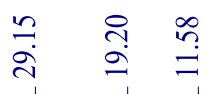

${ }^{13} \mathrm{C} \mathrm{NMR}$ of $1 \mathrm{i}$ in $\mathrm{CDCl}_{3}$

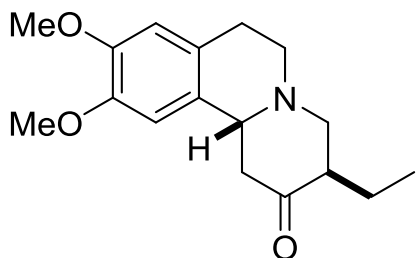

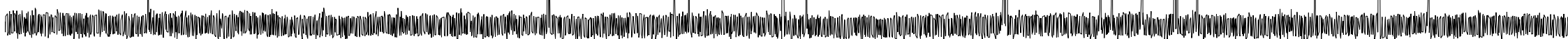

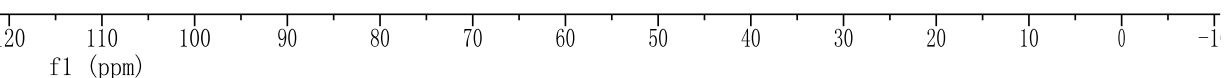




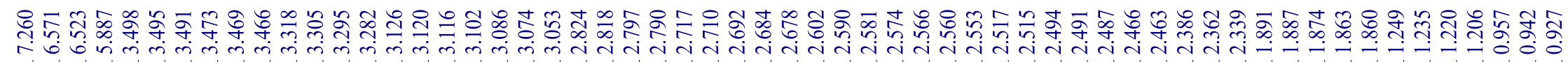
${ }^{1} \mathrm{H}$ NMR of $1 \mathbf{j}$ in $\mathrm{CDCl}_{3}$
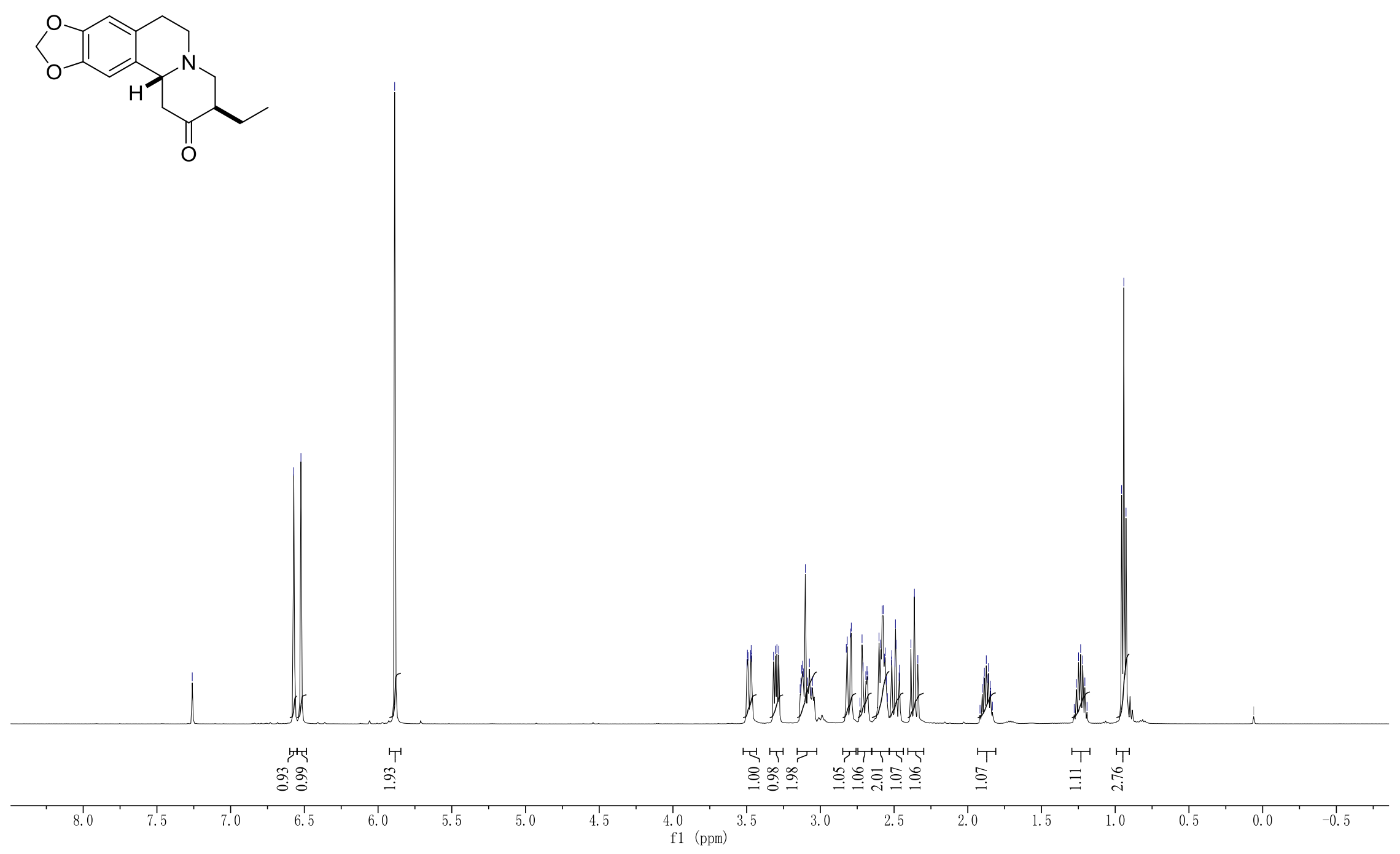

๙े̀

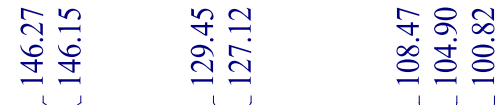

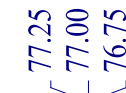
营导
$\stackrel{\text { กิ }}{\stackrel{2}{2}}$

${ }^{13} \mathrm{C} \mathrm{NMR}$ of $\mathbf{1} \mathbf{j}$ in $\mathrm{CDCl}_{3}$

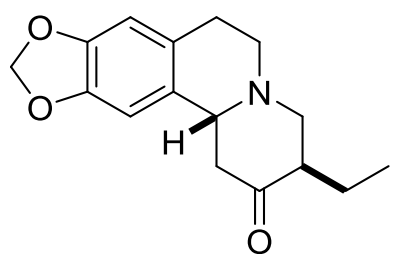

O

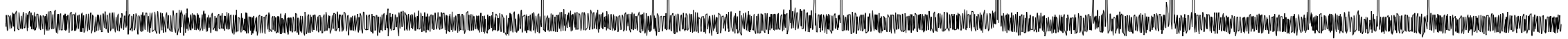

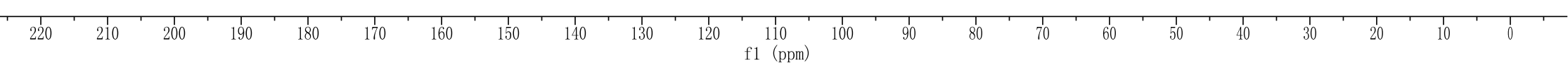




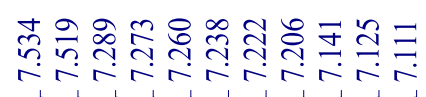

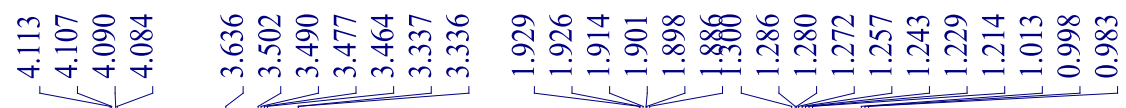

${ }^{1} \mathrm{H}$ NMR of $\mathbf{1} \mathbf{k}$ in $\mathrm{CDCl}_{3}$
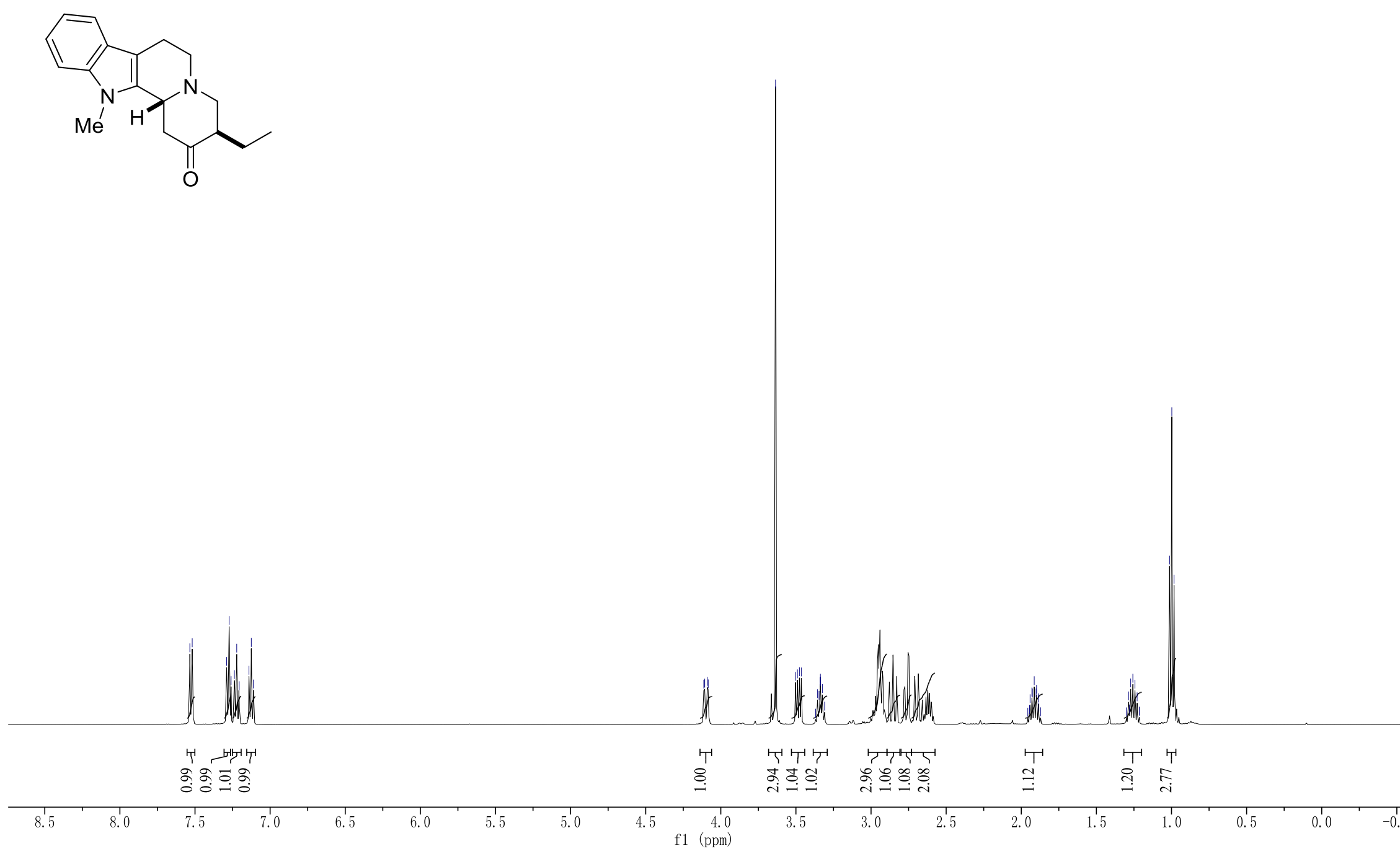

aे
ڤे

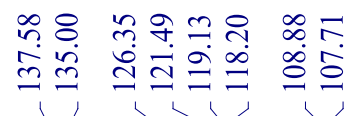

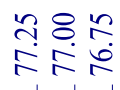

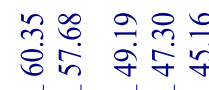

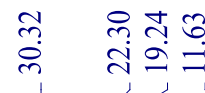

${ }^{13} \mathrm{C} \mathrm{NMR}$ of $\mathbf{1} \mathbf{k}$ in $\mathrm{CDCl}_{3}$
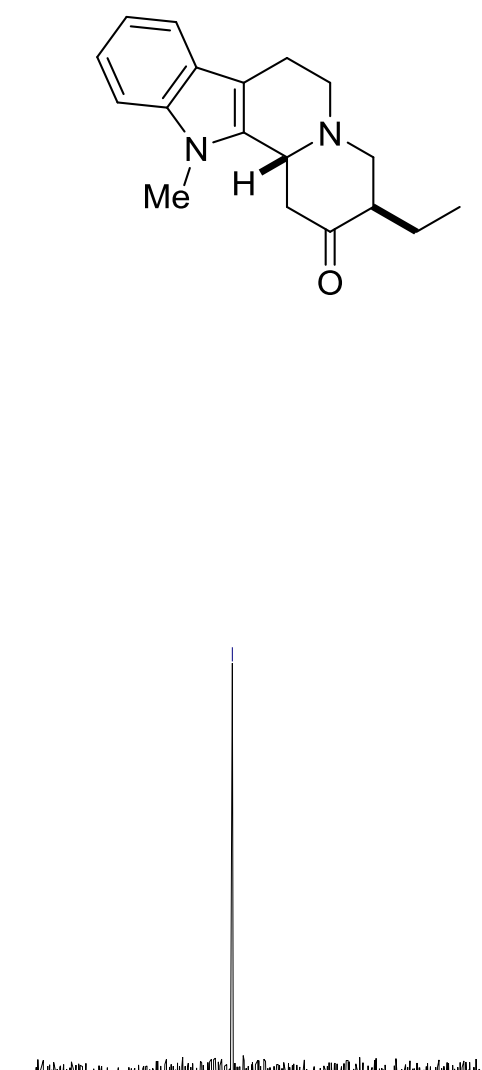

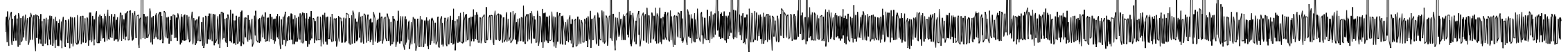
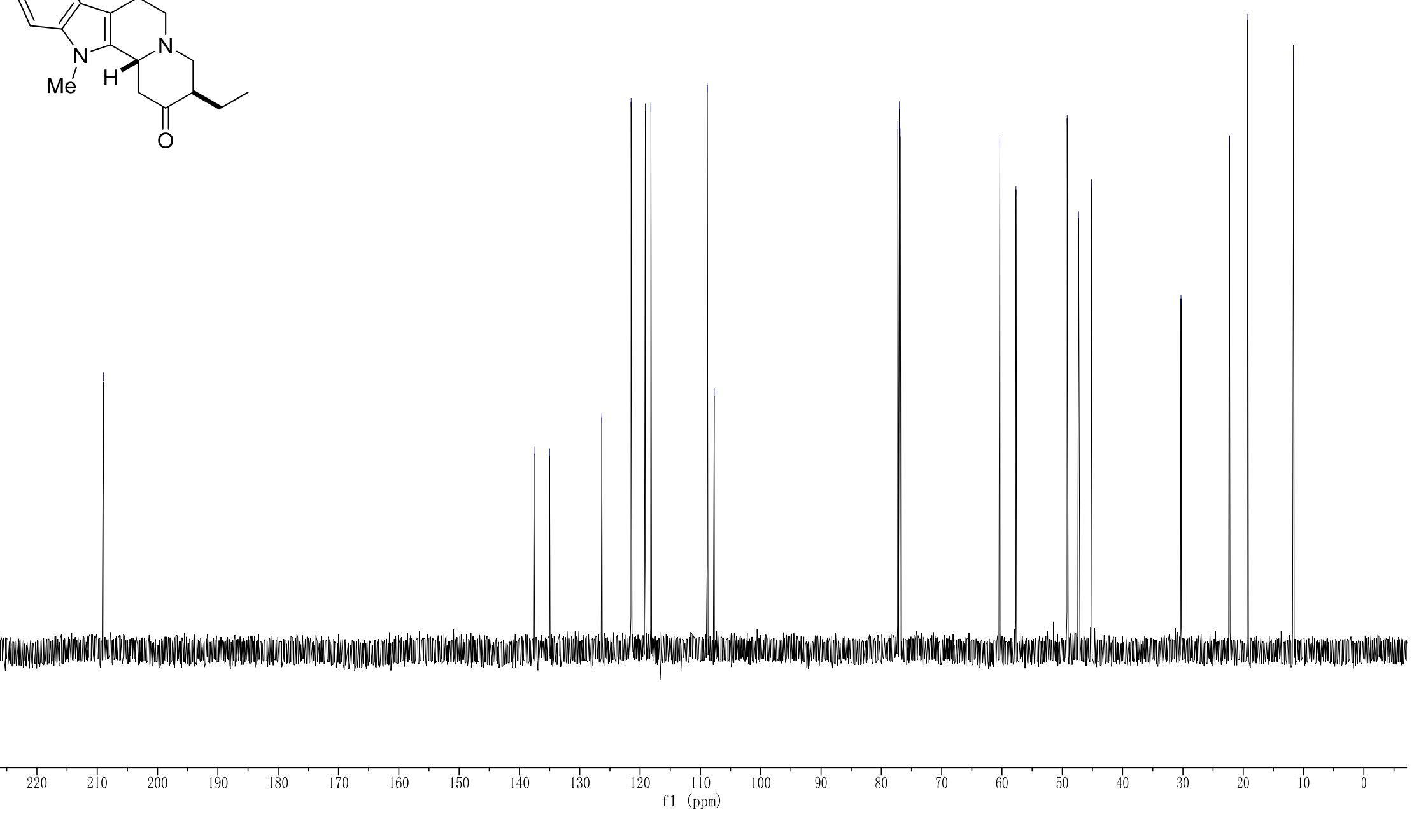


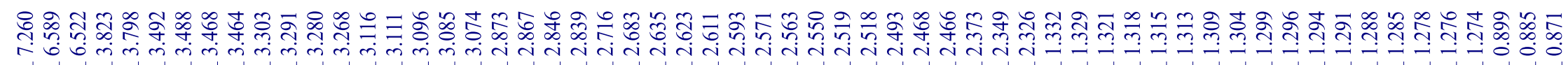

\section{${ }^{1} \mathrm{H} \mathrm{NMR}$ of $1 \mathrm{in} \mathrm{CDCl}_{3}$}
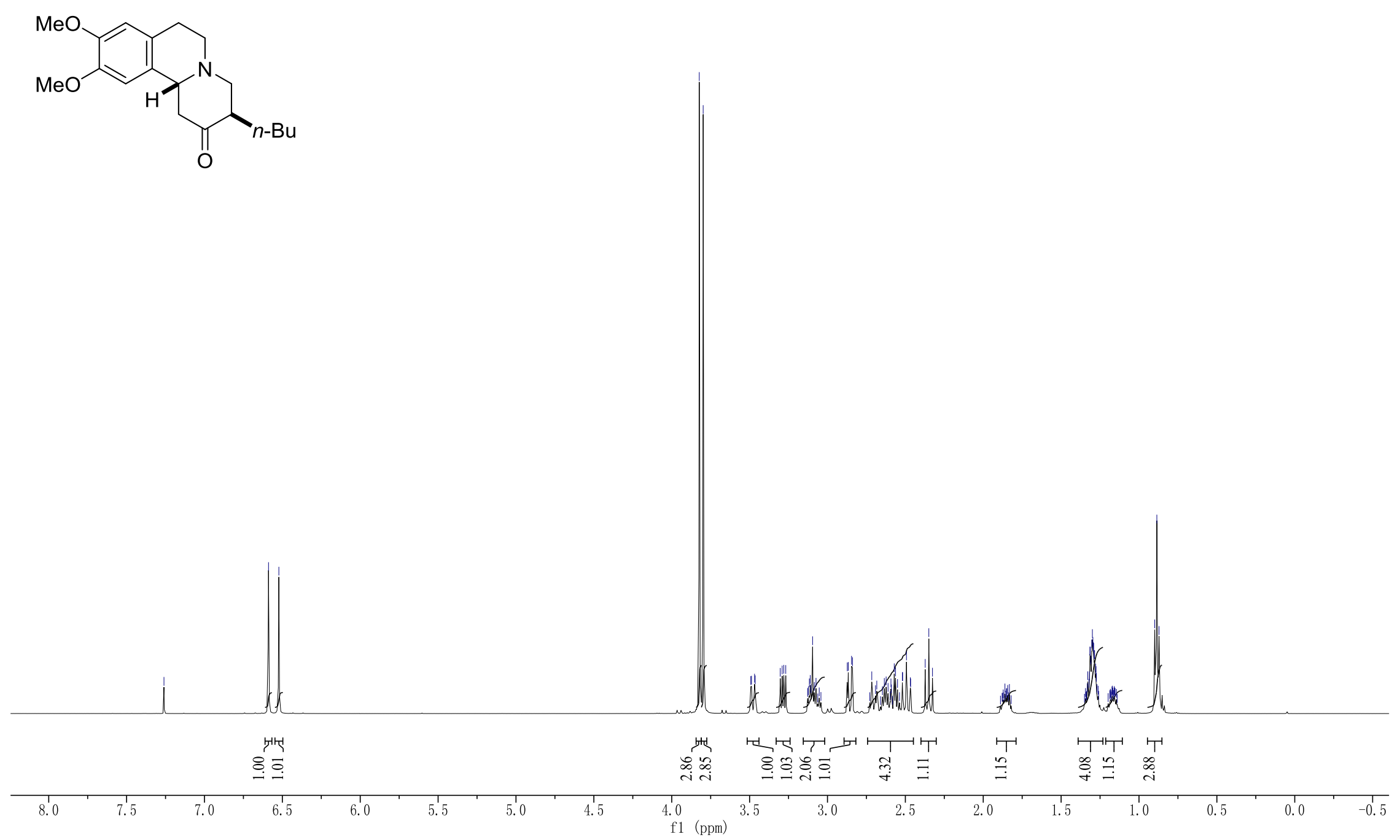

$\stackrel{\infty}{\stackrel{2}{\circ}}$

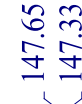
告朵

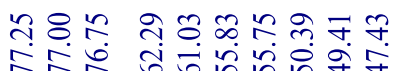

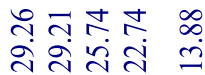

${ }^{13} \mathrm{C} \mathrm{NMR}$ of $1 \mathrm{I}$ in $\mathrm{CDCl}_{3}$

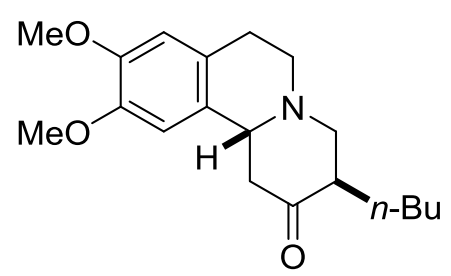

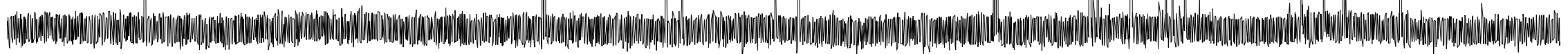

$20 \underset{f 1}{110} 100$ 


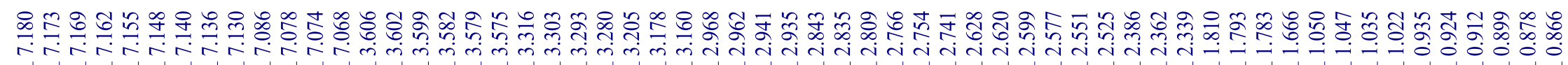
${ }^{1} \mathrm{H}$ NMR of $1 \mathrm{~m}$ in $\mathrm{CDCl}_{3}$
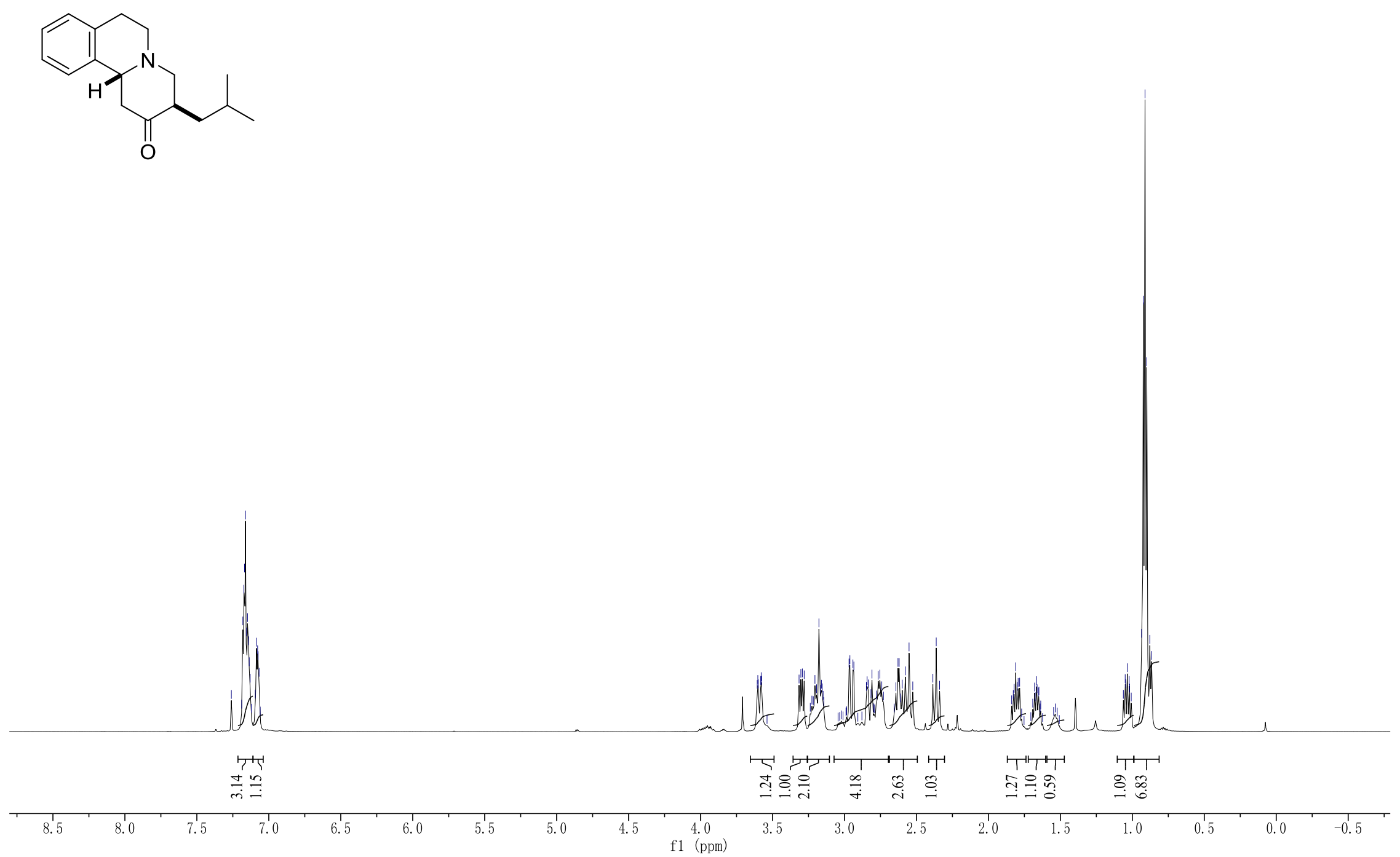


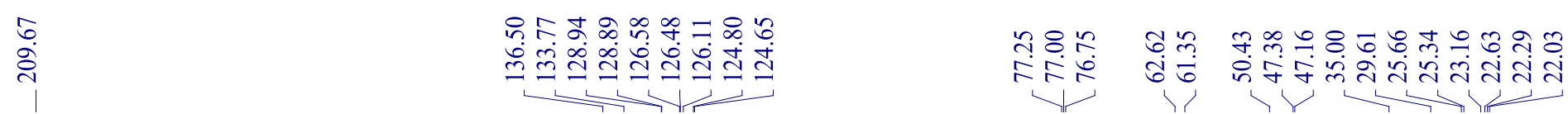

${ }^{13} \mathrm{C} \mathrm{NMR}$ of $1 \mathrm{~m}$ in $\mathrm{CDCl}_{3}$

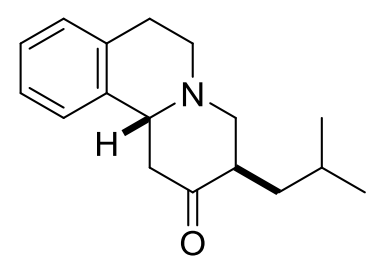

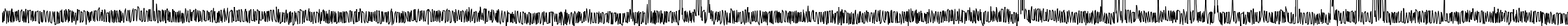

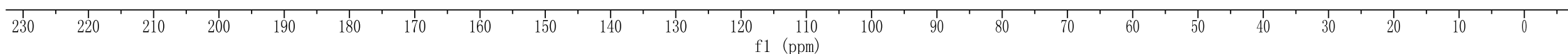




\section{${ }^{1} \mathrm{H}$ NMR of 10 in $\mathrm{CDCl}_{3}$}
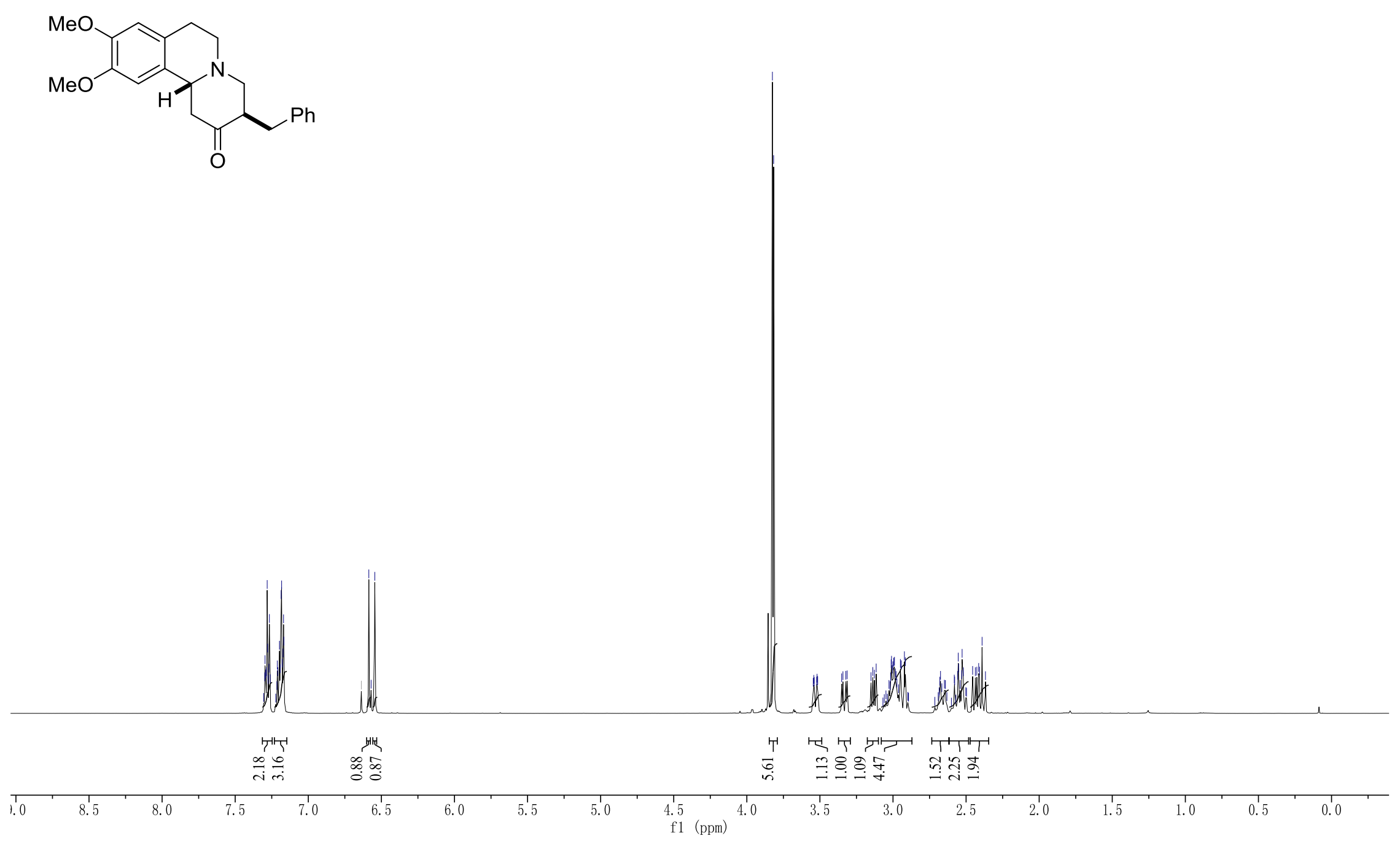

$\infty$
$\stackrel{\infty}{\infty}$
$\stackrel{\Delta}{1}$

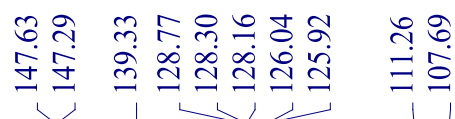

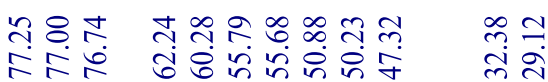

${ }^{13} \mathrm{C} \mathrm{NMR}$ of 10 in $\mathrm{CDCl}_{3}$

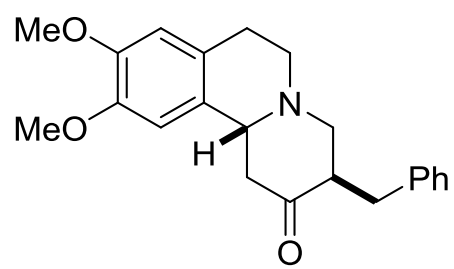

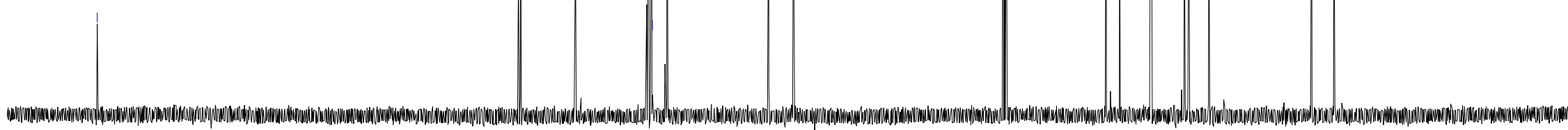

\begin{tabular}{|c|}
\hline 21 \\
\hline
\end{tabular}




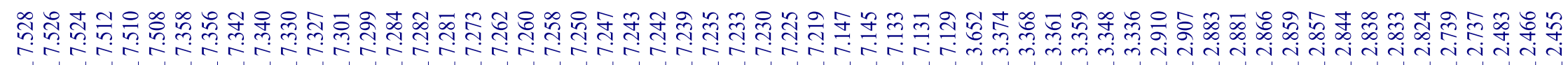
${ }^{1} \mathrm{H}$ NMR of $1 \mathrm{p}$ in $\mathrm{CDCl}_{3}$
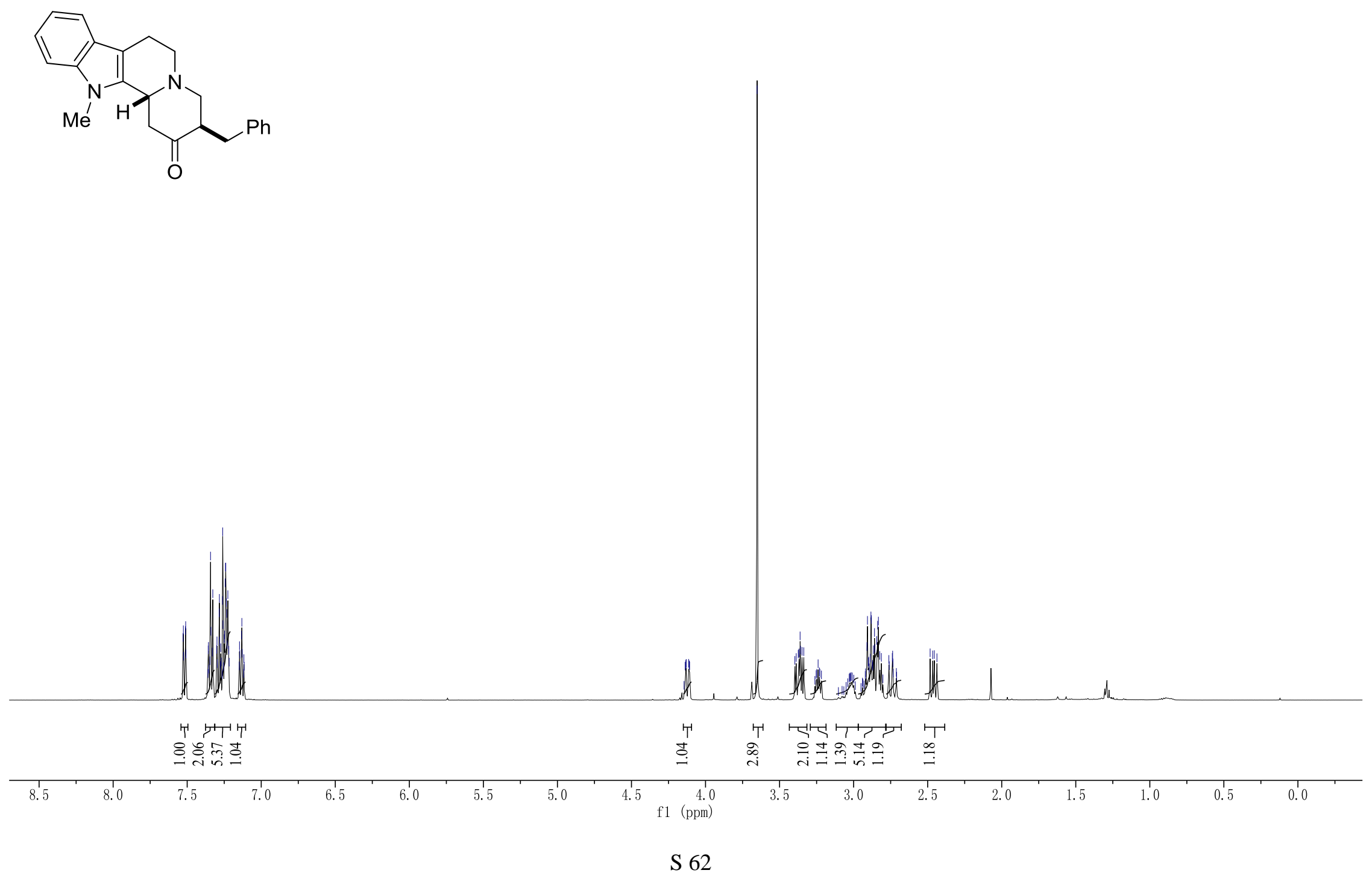


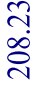
की

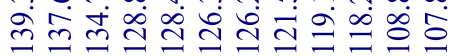
굴욛ำ

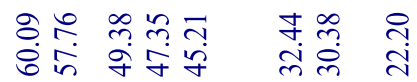

${ }^{13} \mathrm{C} \mathrm{NMR}$ of $1 \mathrm{p}$ in $\mathrm{CDCl}_{3}$
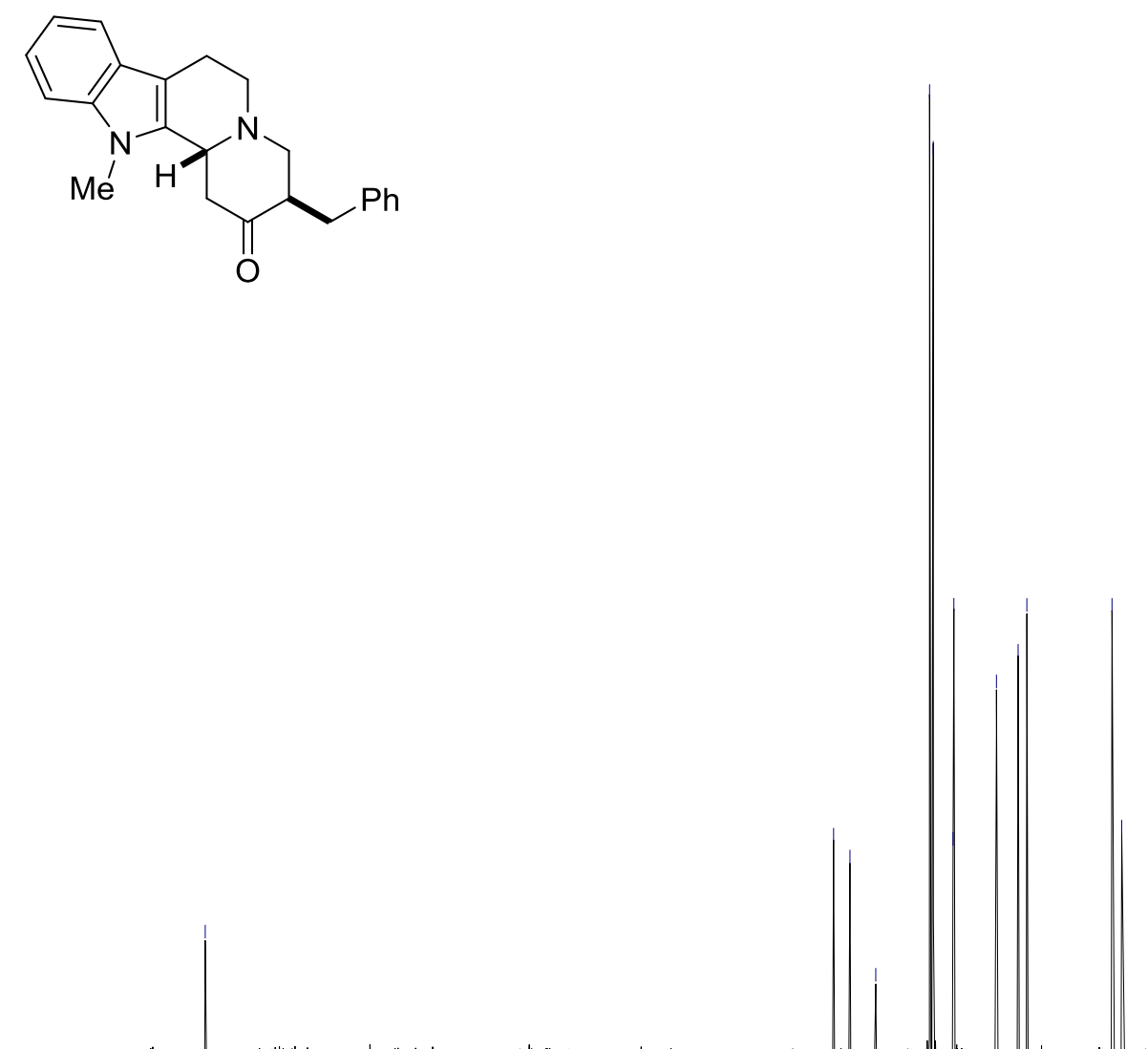

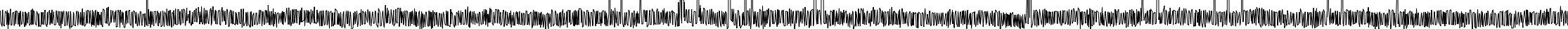

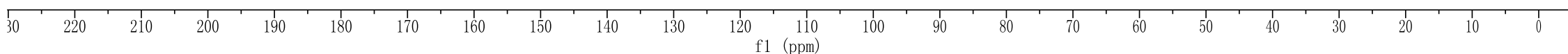




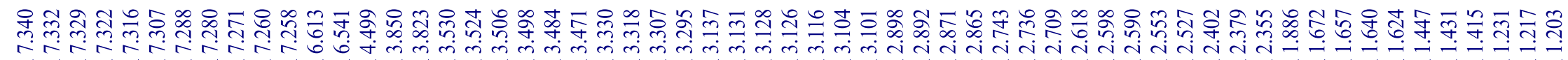

${ }^{1} \mathrm{H}$ NMR of $1 \mathbf{q}$ in $\mathrm{CDCl}_{3}$
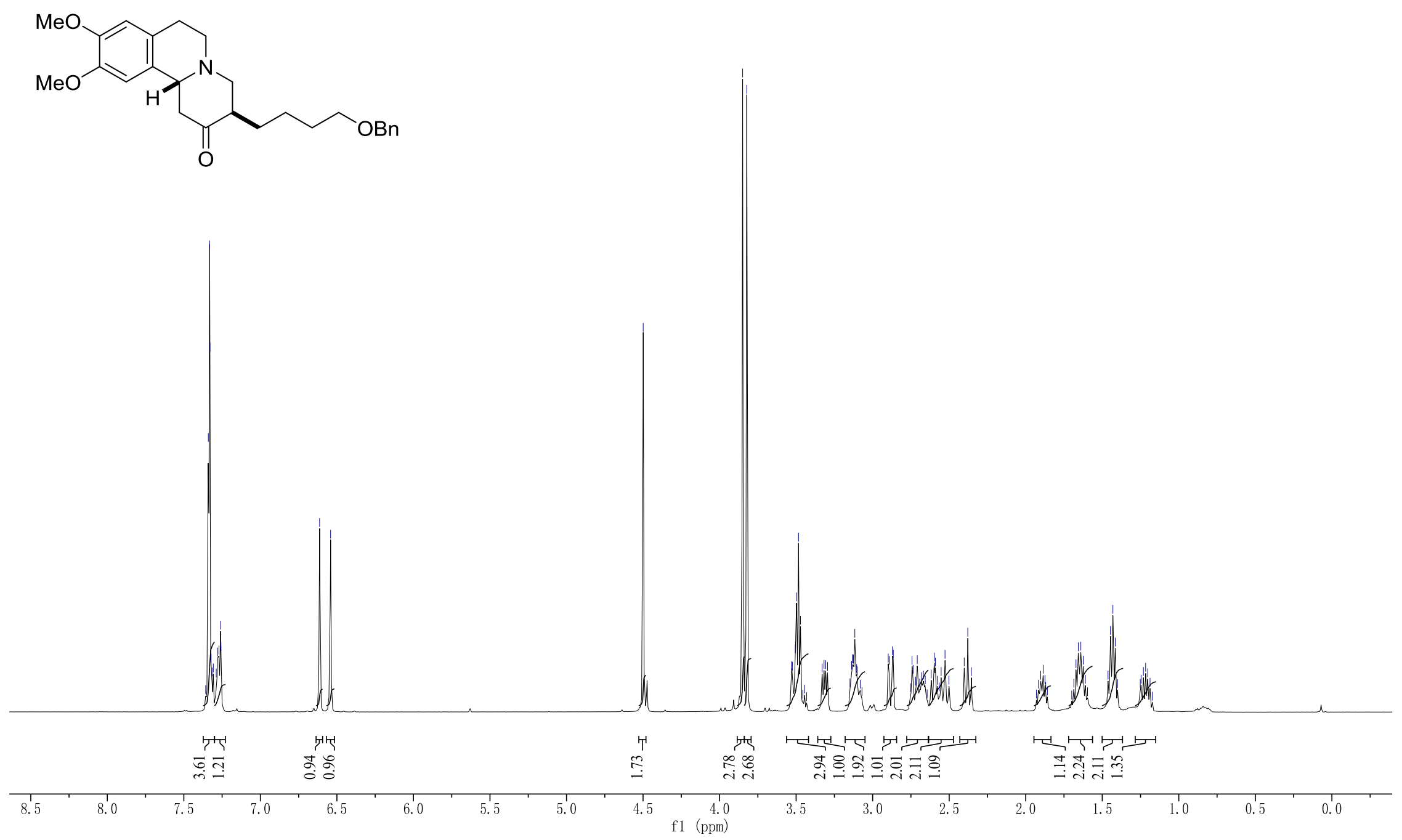
高

${ }^{13} \mathrm{C} \mathrm{NMR}$ of $\mathbf{1 q}$ in $\mathrm{CDCl}_{3}$
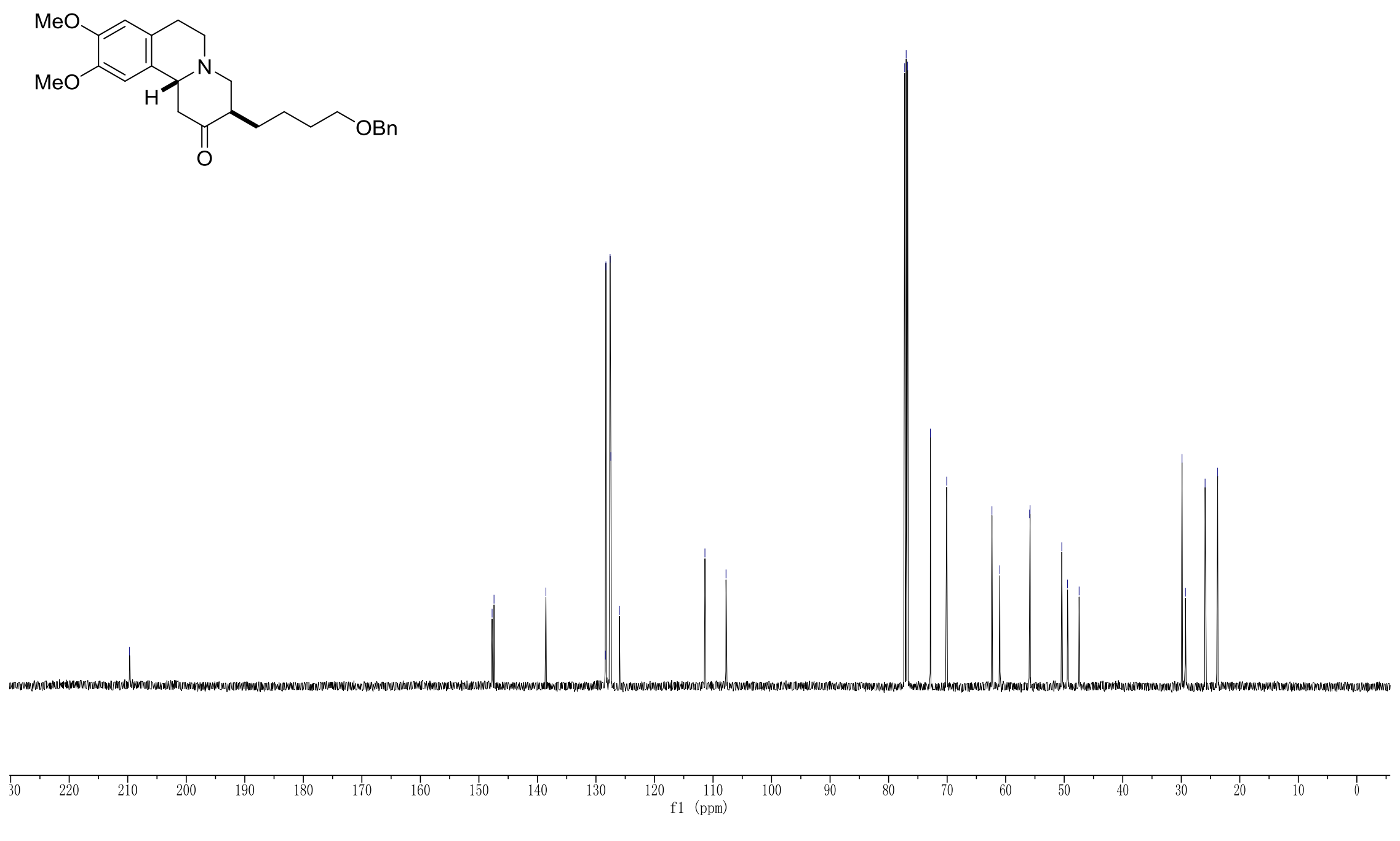


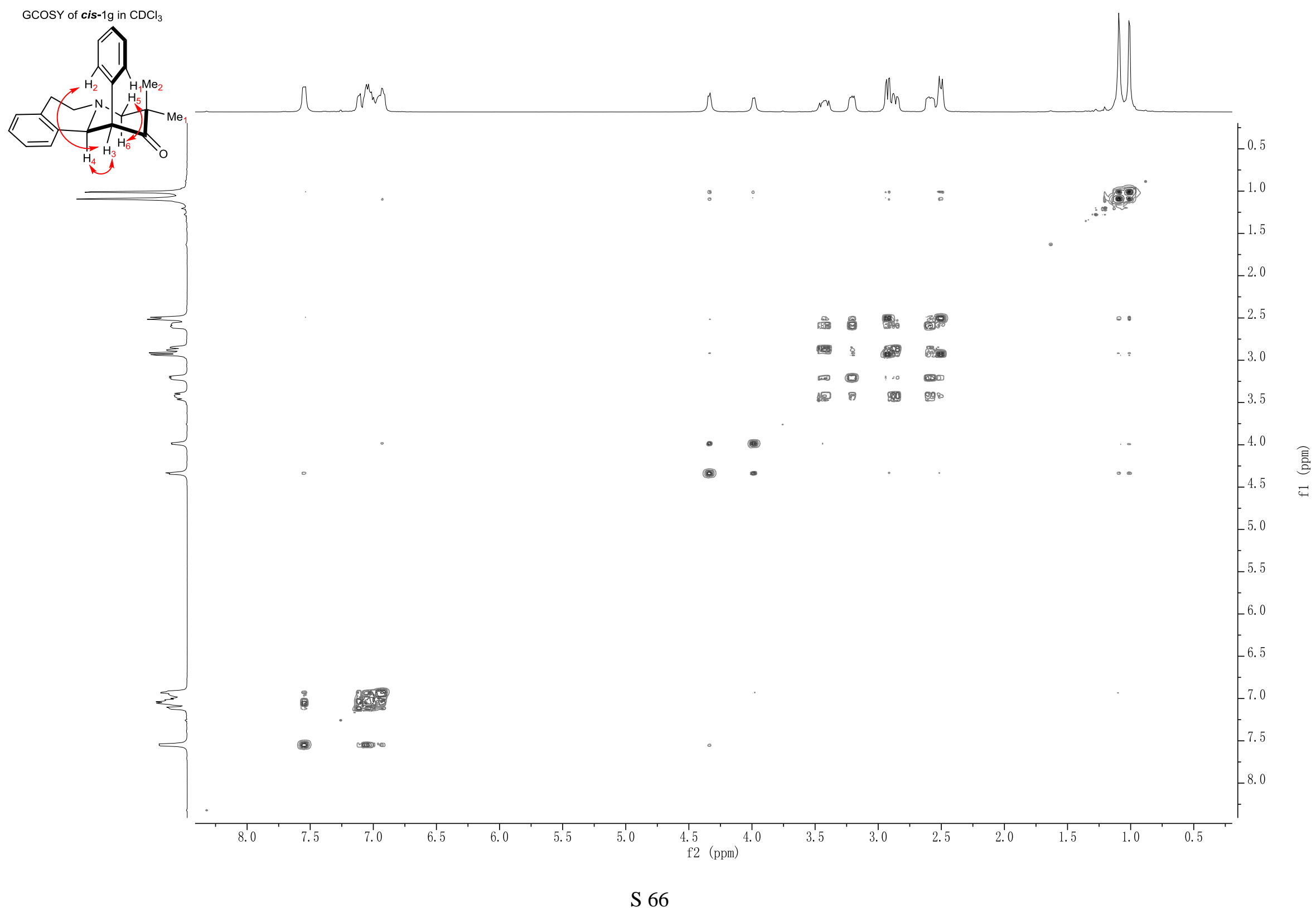




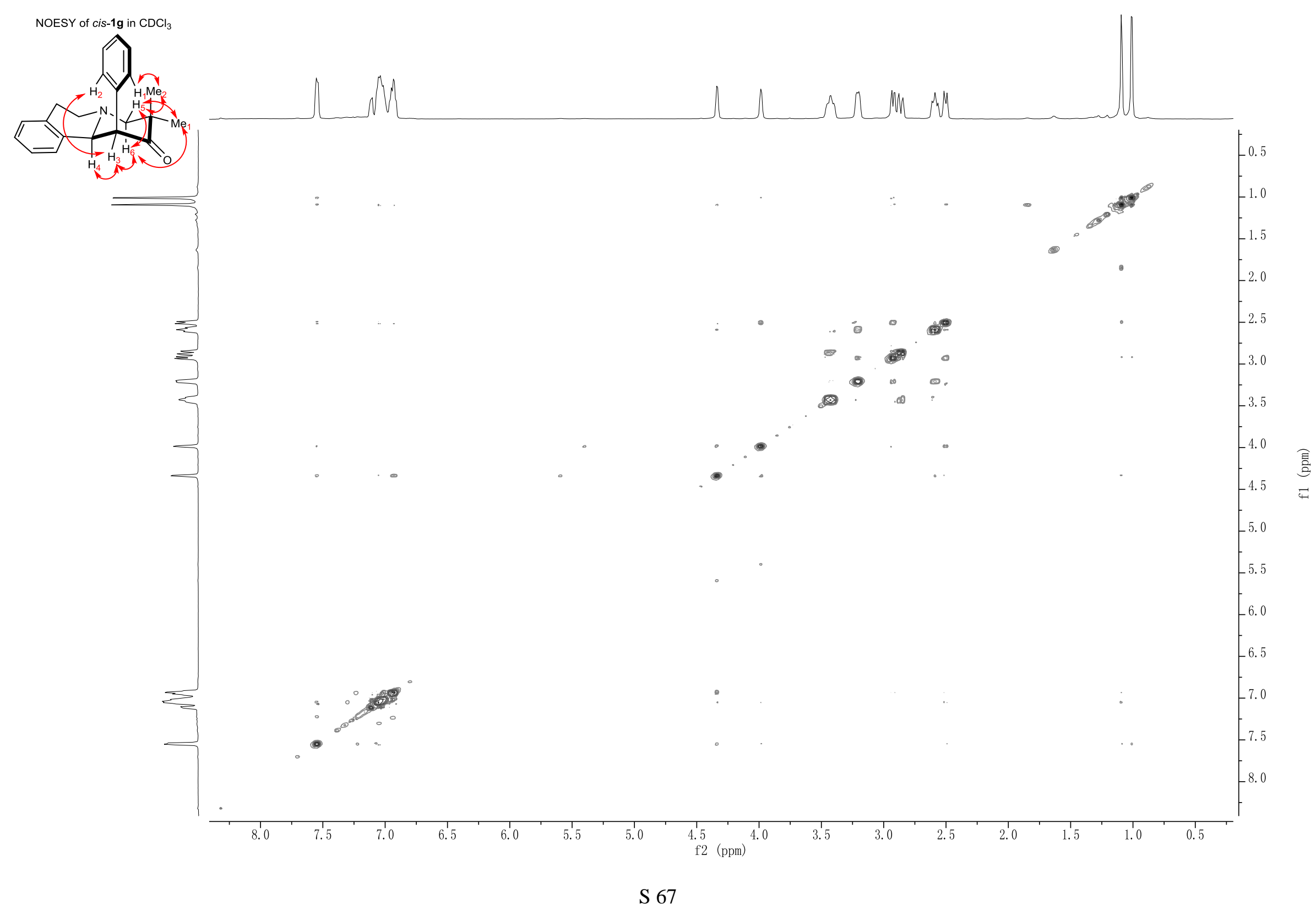

